\title{
Análise de Variância Utilizando Ondaletas
}

Deyvid Toledo Santiago de Almeida

\author{
DISSERTAÇÃO APRESENTADA
}

$\mathrm{AO}$

Instituto DE MATEMÁtica E EstatísticA

$\mathrm{DA}$

Universidade DE SÃo PAUlo

PARA

OBTENÇÃO DO TÍTULO

$\mathrm{DE}$

Mestre em CiÊnCIAS

Programa: Pós-Graduação em Estatística

Orientador: Prof. ${ }^{a}$ Dra. Chang Chiann

Durante o desenvolvimento deste trabalho o autor recebeu auxílio financeiro da CNPq

São Paulo, março de 2020 


\section{Análise de Variância Utilizando Ondaletas}

Esta versão da dissertação contém as correções e alterações sugeridas pela Comissão Julgadora durante a defesa da versão original do trabalho, realizada em 25/06/2020. Uma cópia da versão original está disponível no

Instituto de Matemática e Estatística da Universidade de São Paulo.

Comissão Julgadora:

- Prof. $^{\text {a }}$ Dra. Chang Chiann - IME-USP

- Prof. ${ }^{a}$ Dra. Thelma Sáfadi - UFLA

- Prof. Dr. Michel Helcias Montori - UFSCar 


\section{Resumo}

ALMEIDA, Deyvid T. S. Análise de Variância Utilizando Ondaletas. 2020 Dissertação (Mestrado) - Instituto de Matemática e Estatística, Universidade de São Paulo, São Paulo, 2020.

Análise de Variância no contexto de séries temporais possui a inconveniência da presença de correlação entre as observações. Nessa dissertação, foram estudados métodos de análise de sinais, mais precisamente análise de Fourier e análise de ondaletas (Wavelets), que são ferramentas capazes de transformar o sinal original em uma nova entidade matemática descorrelacionada que possui domínio diferente do original, possibilitando a aplicação da análise de variância sem violar a hipótese de independência dessa metodologia. A diferença mais relevante entre as técnicas é que a análise de Fourier é própria para sinais estacionários, enquanto a análise de ondaletas é robusta a sinais não estacionários pelo fato de sua transformada possuir aspecto local. Na comparação dos resultados por meio de dados simulados, ambas as técnicas convergiram para um mesmo resultado. Para aplicação em dados, reais foram utilizadas medidas de Pico de Fluxo Expiratório (PFE) ao longo do tempo de crianças e adolescentes com condição asmática, ou não, e expostas ao fumo domiciliar, ou não. Na aplicação da ANOVA dois fatores, ambas as metodologias convergiram no teste de interação, mas ocorreram algumas divergências nos testes dos fatores isoladamente.

Palavras-chave: análise de variância em séries temporais, análise de Fourier, análise de ondaletas. 


\section{Abstract}

ALMEIDA, Deyvid T. S. Analysis of Variance using Wavelets. 2020 Dissertation (Master) - Institute of Mathematics e Statistics, University of São Paulo, São Paulo, 2020.

Analysis of Variance in time series context has a correlation problem between observations. In this dissertation, signal analysis methods were studied, more exactly Fourier analysis and wavelet Analysis, which are tools that transform the original signal into a new mathematical entity that has distinct domain from the original, allowing the application of analysis of variance without violating the independence hypothesis of this tool. The most relevant difference between the techniques is that the Fourier analysis is suitable for stationary signals while the wavelet Analysis is robust to non-stationary signals, because its transform has a local aspect. When comparing the results in simulated data, both techniques converged to the same results. For application in real data, measures were taken over the time of Peak Expiratory Flow (PEF) of children and adolescents with or without asthma and exposed or not to smoking environment in their homes. In two-way ANOVA aplication, both techniques converged by the interaction test, but there were some divergent results by factor tests separately.

Keywords: analysis of variance in time series, Fourier analysis, wavelet analysis. 


\section{Sumário}

Lista de Abreviaturas $\quad$ vii

$\begin{array}{ll}\text { Lista de Símbolos } & \text { ix }\end{array}$

Lista de Figuras $\quad$ xiii

Lista de Tabelas $\quad$ xvii

1 Introdução $\quad 1$

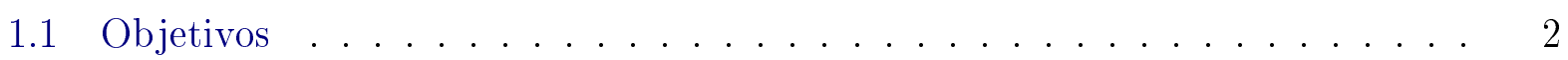

1.2 Organização do Trabalho . . . . . . . . . . . . . . . . 2

2 Conceitos Básicos $\quad 3$

2.1 Definições básicas . . . . . . . . . . . . . . . . . 3

2.2 Propriedades da função de autocovariância . . . . . . . . . . . . . . 4

2.3 Transformações ortonormais . . . . . . . . . . . . . . . . 5

3 Análise clássica de Fourier $\quad 7$

3.1 Análise Espectral de processos estacionários . . . . . . . . . . . 8

3.2 Transformada discreta de Fourier e Periodograma . . . . . . . . . . . . . 9

3.3 Análise de variância usando a transformada discreta de Fourier . . . . . . . . 12

3.3.1 Modelo com um sinal em comum . . . . . . . . . . . . . 14

3.3.2 Modelo com um fator em comum . . . . . . . . . . . . . . 15

3.3.3 Modelo com interação entre dois fatores . . . . . . . . . . . . . 17

4 Análise de ondaletas $\quad 21$

4.1 Análise de Multirresolução . . . . . . . . . . . . . . . . . 23

4.2 Transformada discreta de ondaletas . . . . . . . . . . . . . . 24

4.2.1 Propriedades probabilísticas da transformada discreta de ondaletas . 26

4.2.2 Transformada discreta de ondaletas não decimada . . . . . . . . . . . 27

4.3 Análise de variância usando a transformada discreta de ondaletas . . . . . . 29

4.3.1 Modelo com sinal em comum . . . . . . . . . . . . . . . 29

4.3.2 Modelo com um fator em comum . . . . . . . . . . . . . . 30 
4.3.3 Modelo com interação entre dois fatores . . . . . . . . . . . . 32

5 Simulação $\quad 35$

5.1 Gerando os dados com sinais diferentes . . . . . . . . . . . . . . 35

5.1 .1 Poder do teste . . . . . . . . . . . . . . . . 39

5.2 Gerando os dados com sinais iguais . . . . . . . . . . . . . . . . 41

6 Aplicação $\quad 45$

6.1 Banco de dados . . . . . . . . . . . . . . . . . . . 45

6.2 Análise de variância utilizando Fourier . . . . . . . . . . . . . 46

6.3 Análise de variância utilizando ondaletas . . . . . . . . . . . . . 53

6.3.1 Transformada discreta de ondaletas . . . . . . . . . . . . . . 53

6.3.2 ANOVA utilizando a transformada discreta de ondaletas não decimada 58

$\begin{array}{lll}7 & \text { Conclusões } & 67\end{array}$

A Dados utilizados na aplicação $\quad 69$

Referências Bibliográficas $\quad 73$ 


\title{
Lista de Abreviaturas
}

\author{
TDF Transformada discreta de Fourier \\ TDO Transformada discreta de ondaletas \\ TDOND Transformada discreta de ondaletas não decimada \\ ANOVA Análise de variância (Analysis of variance) \\ SQ Soma de quadrados \\ QM Quadrado médio
}


viii LISTA DE ABREVIATURAS 


\section{Lista de Símbolos}

$\tau \quad$ Conjunto de tempo discreto

$t \quad$ Elemento pertencente a $\tau$

$X(t)$ Variável aleatória indexada por $t$

$F \quad$ Função de distribuição acumulada

$P \quad$ Probabilidade

$\mathbb{E} \quad$ Valor esperado

$\gamma \quad$ Função de autocovariância

Var Variância

$\rho \quad$ Função de autocorrelação

$f_{\mathbf{x}}() \quad$ Função de densidade de probabilidade do vetor $\mathbf{x}$

$\Sigma \quad$ Matriz de variâncias e covariâncias

$\mu \quad$ Vetor de médias

$N_{p} \quad$ Distribuição normal $p$-variada

$N_{p}^{c} \quad$ Distribuição normal complexa $p$-variada

$\stackrel{d}{\longrightarrow}$ Convergência em distribuição

$\langle$,$\rangle \quad Produto interno$

|l $\|$ Norma

$\mathcal{P} \quad$ Matriz ortonormal genérica

$\mathcal{P}_{\bullet j} \quad j$-ésima coluna da matriz $\mathcal{P}$

$\mathcal{P}_{k} \bullet \quad k$-ésima linha da matriz $\mathcal{P}$

$\mathbf{P} \quad$ Matriz de coeficientes da transformação ortogonal

$\hat{\mathbf{X}} \quad$ Projeção do vetor $\mathbf{X}$

$\beta \quad$ Coeficiente do vetor $\mathbf{P}$

$\mathcal{I} \quad$ Matriz identidade

$\lambda \quad$ Frequência

$F_{n} \quad$ Coeficiente da transformada discreta de Fourier indexado por $n$

$J(\lambda) \quad$ Função de densidade de potência

$f(\lambda) \quad$ Função de densidade espectral

$d_{\lambda} \quad$ Coeficiente da transformada finita de fourier na frequência $\lambda$

$I_{\lambda} \quad$ Periodograma na frequência $\lambda$

$\chi^{2} \quad$ Distribuição qui-quadrado

$\hat{\gamma} \quad$ Função de autocovariância amostral

$w \quad$ Função núcleo

$W \quad$ Janela espectral 
$\bar{I}_{\lambda} \quad$ Periodograma suavizado de Bartlett

$\hat{f} \quad$ Estimador suavizado de covariâncias

$\tilde{f}$ Estimador suavizado de periodogramas

$\tilde{I}_{\lambda} \quad$ Periodograma suavizado de Daniell 


\section{Lista de Figuras}

4.1 (1) Haar; (2) Mexican Hat; (3) Extremal Phase D4; (4) Least Asymmetric LA4. 22

4.2 Função de autocorrelação típica da TDOND após e antes da aplicação da transformação 4.40. . . . . . . . . . . . . . . . . . 29

5.1 Sinais das séries simuladas. . . . . . . . . . . . . . . 35

5.2 Simulação TDO: $p$-valores exibidos por nível de resolução $J=8,7,6,5,4,3$. . 36

5.3 Simulação TDO: $p$-valores exibidos por nível de resolução $J=2,1,0$. . . 37

5.4 Simulação TDOND: $p$-valores exibidos por nível de resolução. . . . . . . . . 38

5.5 Sinais diferentes: $p$-valores da transformada de Fourier. . . . . . . . . . . . 39

5.6 Poder do teste empírico para transformada discreta de ondaletas nas resoluções $J=8,7,6,5,4,3 \ldots \ldots \ldots \ldots \ldots \ldots \ldots$

5.7 Simulação TDO: Poder do teste empírico para transformada discreta de ondaletas nas resoluções $J=2,1,0 \ldots \ldots \ldots$. . . . . . . . . 40

5.8 Simulação TDOND: Poder do teste empírico para transformada discreta de ondaletas não decimada. . . . . . . . . . . . . . . . . . . 40

5.9 simulação TDF: Poder do teste empírico para transformada discreta de Fourier. 41

5.10 Simulação TDO: $p$-valores exibidos por nível de resolução $J=8,7,6,5,4,3$. 42

5.11 Simulação TDO: $p$-valores exibidos por nível de resolução $J=2,1,0$. . . . 42

5.12 Simulação TDOND: $p$-valores exibidos por nível de resolução. . . . . . . . . 43

5.13 Simulação TDF: $p$-valores da transformada de Fourier. . . . . . . . . . . 43

5.14 Histograma das estatísticas de teste observadas junto com a distribuição teórica (TDO na parte superior esquerda, TDOND na parte superior direita e

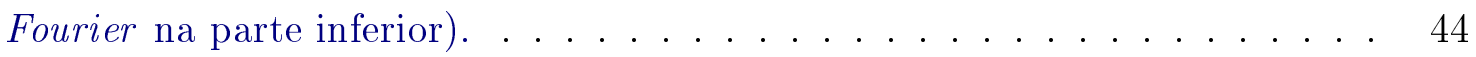

6.1 Amostra das séries dos indivíduos sem a condição de asma e não expostos ao fumo domiciliar. . . . . . . . . . . . . . . . . . 46

6.2 Amostra das séries dos indivíduos com a condição de asma e não expostos ao fumo domiciliar. . . . . . . . . . . . . . . . . . . . . . . .

6.3 Amostra das séries dos indivíduos sem a condição de asma e expostos ao fumo domiciliar. . . . . . . . . . . . . . . . 48

6.4 Amostra das séries indivíduos com a condição de asma e expostos ao fumo

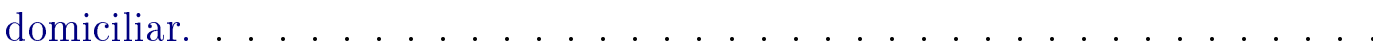


6.5 TDF: p-valores dos testes de interação para cada frequência $\lambda(k) . \ldots$. . . .

6.6 TDF: p-valores dos testes do efeito da asma nos expostos para cada frequência

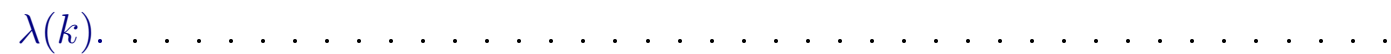

6.7 TDF: p-valores dos testes do efeito da asma nos não expostos para cada frequência $\lambda(k) \ldots \ldots \ldots \ldots \ldots$

6.8 TDF: p-valores dos testes de sinal comum nos expostos e asmáticos para cada frequência $\lambda(k) \ldots \ldots \ldots \ldots \ldots \ldots \ldots \ldots \ldots \ldots \ldots \ldots \ldots \ldots \ldots \ldots$

6.9 TDF: p-valores dos testes de sinal comum nos expostos e não asmáticos para cada frequência $\lambda(k) \ldots \ldots \ldots \ldots \ldots$

6.10 TDF: p-valores dos testes de sinal comum nos não expostos e asmáticos para cada frequência $\lambda(k) \ldots \ldots \ldots \ldots \ldots$

6.11 TDF: p-valores dos testes de sinal comum nos não expostos e não asmáticos para cada frequência $\lambda(k)$.

6.12 TDO: p-valores dos testes de interação para cada índice $(j, k)$. Extremal Phase (D8) a esquerda e Least Asymmetric (LA8) a direita. . . . . . . . . . . . .

6.13 TDO: p-valores dos testes do efeito da asma nos expostos para cada índice $(j, k)$. Extremal Phase (D8) a esquerda e Least Asymmetric (LA8) a direita.

6.14 TDO: p-valores dos testes do efeito da asma nos não expostos para cada índice $(j, k)$. Extremal Phase (D8) a esquerda e Least Asymmetric (LA8) a direita.

6.15 TDO: p-valores dos testes de sinal em comum nos expostos e asmáticos para cada índice $(j, k)$. Extremal Phase (D8) a esquerda e Least Asymmetric (LA8) a direita. . . . . . . . . . . . . . . . . . .

6.16 TDO: p-valores dos testes de sinal em comum nos expostos e não asmáticos para cada índice $(j, k)$. Extremal Phase (D8) a esquerda e Least Asymmetric

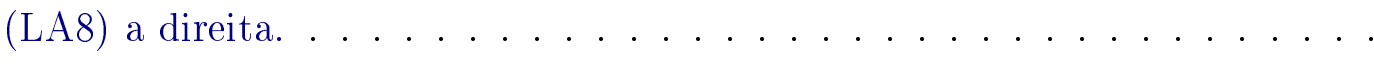

6.17 TDO: p-valores dos testes de sinal em comum nos não expostos e asmáticos para cada índice $(j, k)$. Extremal Phase (D8) a esquerda e Least Asymmetric (LA8) a direita. . . . . . . . . . . . . . . . . . .

6.18 TDO: p-valores dos testes de sinal em comum nos não expostos e não asmáticos para cada índice $(j, k)$. Extremal Phase (D8) a esquerda e Least Asymmetric (LA8) a direita. . . . . . . . . . . . . .

6.19 TDOND: p-valores dos testes de interação para cada índice $(j, k)$. Extremal Phase (D8) a esquerda e Least Asymmetric (LA8) a direita. . . . . . . . .

6.20 TDOND: p-valores dos testes do efeito da asma nos expostos para cada índice $(j, k)$. Extremal Phase (D8) a esquerda e Least Asymmetric (LA8) a direita.

6.21 TDOND: p-valores dos testes do efeito da asma nos não expostos para cada índice $(j, k)$. Extremal Phase (D8) a esquerda e Least Asymmetric (LA8) a direita. . . . . . . . . . . . . . . . . . . . 
6.22 TDOND: p-valores dos testes de sinal em comum nos expostos e asmáticos para cada índice $(j, k)$. Extremal Phase (D8) a esquerda e Least Asymmetric (LA8) a direita. . . . . . . . . . . . . . . . . . 64

6.23 TDOND: p-valores dos testes de sinal em comum nos expostos e não asmáticos para cada índice $(j, k)$. Extremal Phase (D8) a esquerda e Least Asymmetric $(\mathrm{LA} 8)$ a direita. . . . . . . . . . . . . . . . . . 64

6.24 TDOND: p-valores dos testes de sinal em comum nos não expostos e asmáticos para cada índice $(j, k)$. Extremal Phase (D8) a esquerda e Least Asymmetric (LA8) a direita. . . . . . . . . . . . . . . . .

6.25 TDOND: p-valores dos testes de sinal em comum nos não expostos não asmáticos para cada índice $(j, k)$. Extremal Phase (D8) a esquerda e Least Asymmetric (LA8) a direita. . . . . . . . . . . . . . 65

A.1 Séries dos indivíduos sem a condição de asma e não expostos ao fumo domiciliar. 69

A.2 Séries dos indivíduos com a condição de asma e não expostos ao fumo domiciliar. 70

A.3 Séries dos indivíduos sem a condição de asma e expostos ao fumo domiciliar. 70

A.4 Séries dos indivíduos com a condição de asma e expostos ao fumo domiciliar. 71 


\section{Lista de Tabelas}

3.1 ANOVA para testar diferença de sinais em comum na frequência $\lambda_{k} \ldots \ldots 15$

3.2 ANOVA para testar diferença de sinais determinísticos de tratamentos na frequência $\lambda_{k} \ldots \ldots \ldots \ldots \ldots \ldots$. . . . . . . . . . . . . . . . . . .

3.3 ANOVA para testar sinal determinístico de interação entre grupos de tratamentos na frequência $\lambda_{k} \ldots \ldots \ldots \ldots$

4.1 ANOVA para testar diferença de sinais em comum para índices $(j, k) \ldots \ldots$

4.2 ANOVA para testar diferença de sinais determinísticos de tratamentos nos índices $(j, k) \ldots \ldots \ldots \ldots \ldots \ldots \ldots \ldots \ldots \ldots \ldots \ldots \ldots \ldots \ldots \ldots \ldots \ldots$

4.3 ANOVA para testar sinal determinístico de interação entre grupos de tratamentos nos índices $(j, k) \ldots \ldots \ldots \ldots$. . . . . . . . . . . 34

6.1 TDF: p-valores dos testes de interação para cada frequência $\lambda(k)$, frequências significativas estão em destaque. . . . . . . . . . . . . . . . . 47

6.2 TDF: p-valores dos testes do efeito da asma nos expostos para cada frequência $\lambda(k) \ldots \ldots \ldots \ldots \ldots \ldots \ldots$

6.3 TDF: p-valores dos testes do efeito da asma nos não expostos para cada frequência $\lambda(k) \ldots \ldots \ldots \ldots \ldots \ldots$

6.4 TDO: p-valores dos testes de interação para cada índice $(j, k)$, índices significativos estão em destaque. . . . . . . . . . . . . . 54

6.5 TDO: p-valores dos testes do efeito da asma nos expostos para cada índice

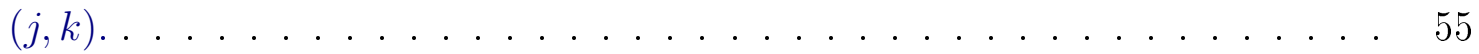

6.6 TDO: p-valores dos testes do efeito da asma nos não expostos para cada índice

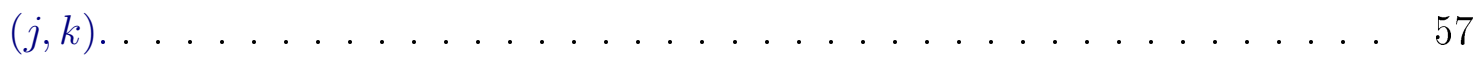

6.7 TDOND: p-valores dos testes de interação para cada índice $(j, k) . \ldots \ldots 1$

6.8 TDOND: p-valores dos testes do efeito da asma nos expostos para cada índice

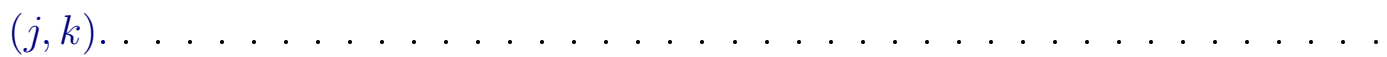

6.9 TDOND: p-valores dos testes do efeito da asma nos não expostos para cada

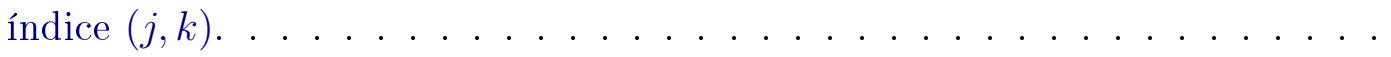




\section{Capítulo 1}

\section{Introdução}

Apesar da análise de ondaletas (Wavelets) não ter nascido com fins estatísticos, mas sim de campos como Engenharia e Física (Abramovich, 2000), a teoria de ondaletas pode ser facilmente abordada em problemas que envolvam Estatística, por exemplo, em situações onde exista alguma dependência nos dados segundo alguma característica natural que os correlacione, como tempo ou distância. A transformada de ondaletas traz informação por meio de representações matemáticas de distintos tipos de objetos, por exemplo, funções, sinais ou imagens. A transformada de ondaletas pode ser unidimensional, bidimensional ou tridimensional e todas essas representações podem ser obtidas por meio de algoritmos extremamente eficientes. Na área da estatística a análise de ondaletas também tem sido usada para tarefas que vão de regressão não paramétrica a estimação de densidades.

A primeira ferramenta conhecida para análise de sinais foi a análise de Fourier, utilizada para estimar a magnitude dos componentes harmônicos. Embora a transformada de Fourier seja adequada para sinais estacionários, ela também é usada em sinais não estacionários, produzindo resultados com ruídos. A transformada de Fourier é realizada por meio de uma base formada por funções de senos e cossenos e possui interpretação de frequência, características que evidenciam a necessidade de estacionariedade. Diferente da primeira, a transformada de ondaletas é realizada por meio de bases formadas de pequenas ondas que possuem média zero, rapidamente decaem para zero e possui interpretação no domínio do tempo e da escala, o que ajuda a entender o porque ela é tão eficiente quando se trata de séries não estacionárias.

A análise de variância clássica, mais conhecida como ANOVA, tem como objetivo verificar a existência entre igualdade de médias de duas ou mais populações. No contexto de séries temporais, a análise de variância pode ser vista com o objetivo de verificar a existência de diferença no sinal entre dois ou mais grupos de processos estocásticos, no entanto, a aplicação da análise de variância clássica, em geral, não cumpre todos os requisitos teóricos no caso de séries temporais, pois é difícil supor independência em uma dada série de variáveis aleatórias. Por isso, é necessário lançar mão de técnicas mais rebuscadas, como a análise espectral. O uso de ferramentas como a análise de Fourier são bastante úteis, principalmente para séries ditas estacionárias, devido à independência assintótica obtida por meio da transformada discreta de Fourier. Contudo, a ausência de estacionariedade acarreta em menor efetividade no uso da análise de Fourier. Nos casos em que não há estacionariedade é preciso uma maneira mais geral para realizar outras transformações que melhor se adaptem a esse fenômeno. Nessa dissertação será abordada a análise de wavelets, através do uso da transformada discreta de wavelets, que é vista como uma alternativa à análise de Fourier, mas sem restrições de estacionariedade. 


\section{$1.1 \quad$ Objetivos}

O objetivo dessa dissertação é de elucidar melhor o tema de análise de variância em séries temporais, que é ainda muito escasso na literatura, principalmente quando é abordado conjuntamente com Análise de Ondaletas.

\subsection{Organização do Trabalho}

No capítulo 2 são apresentados conceitos básicos para análise de Fourier e Ondaletas. No capítulo 3, são apresentados os conceitos na análise de Fourier, sua transformada discreta e a análise de variância no contexto de Fourier. No capítulo 4, abordamos a análise de ondaletas, sua transformada e análise de variância em seu contexto. No capítulo 5, por meio de simulação, os desempenhos dos dois métodos na aplicação de ANOVA foram comparados. No capítulo 6 aplicamos os dois métodos de análise de variância em dados reais. Finalmente, no capítulo 7 discutimos os resultados obtidos pelos diferentes métodos e algumas limitações encontradas na aplicação de dados reais. 


\section{Capítulo 2}

\section{Conceitos Básicos}

Nesse capítulo, serão introduzidos alguns conceitos básicos de processos estocásticos e transformações ortogonais, que são a base para o entendimento da Análise de Fourier e da análise de ondaletas (Wavelets) (Percival e Walden, 2000), (Morettin, 2014).

\subsection{Definições básicas}

Definição 2.1: Seja $\tau$ um conjunto arbitrário. Um processo estocástico é uma família $\{X(t), t \in \tau\}$, tal que, para cara $t \in \tau, X(t)$ é uma variável aleatória.

O conjunto $\tau$ usualmente é considerado como o conjunto dos números reais $\mathbb{R}$ ou inteiros $\mathbb{Z}$. Nesse trabalho, o conjunto $\tau$ será considerado como o conjunto dos números inteiros $\mathbb{Z}$. Além disso, o processo estocástico assumirá valores no conjunto dos reais $\mathbb{R}$.

Definição 2.2: Seja $t_{1}, \ldots, t_{n}$ elementos quaisquer de $\tau$, as distribuições finito-dimensionais do processo estocástico $\{X(t), t \in \tau\}$ são definidas por:

$$
F\left(x_{1}, \ldots, x_{n} ; t_{1}, \ldots, t_{n}\right)=P\left\{X\left(t_{1}\right) \leq x_{1}, \ldots, X\left(t_{n}\right) \leq x_{n}\right\} .
$$

Definição 2.3: Seja $\{X(t), t \in \tau\}$ um processo estocástico. O valor esperado de $X(t)$, ou seja, a média do processo no instante $t$ é dado por:

$$
\mathbb{E}\{X(t)\}=\int_{-\infty}^{\infty} x d F(x ; t) .
$$

Definição 2.4: Seja $\{X(t), t \in \tau\}$ um processo estocástico. A função de autocovariância do processo nos instantes $t_{1}, t_{2}$ é definida como:

$\gamma\left(t_{1}, t_{2}\right)=\mathbb{E}\left\{\left[X\left(t_{1}\right)-\mathbb{E}\left\{X\left(t_{1}\right)\right\}\right]\left[X\left(t_{2}\right)-\mathbb{E}\left\{X\left(t_{2}\right)\right\}\right]\right\}=\mathbb{E}\left\{X\left(t_{1}\right) X\left(t_{2}\right)\right\}-\mathbb{E}\left\{X\left(t_{1}\right)\right\} \mathbb{E}\left\{X\left(t_{2}\right)\right\}$

Quando consideramos $t_{1}=t_{2}=t$ obtemos a variância do processo no instante $t$,

$$
\operatorname{Var}\{X(t)\}=\gamma(t, t)=\mathbb{E}\left\{X(t)^{2}\right\}-\mathbb{E}\{X(t)\}^{2} .
$$

Definição 2.5: Um processo estocástico $\{X(t), t \in \tau\}$ é dito estritamente estacionário se todas as distribuições finito dimensionais permanecem as mesmas sob translações no tempo, isto é,

$$
F\left(x_{1}, \ldots, x_{n} ; t_{1}, \ldots, t_{n}\right)=F\left(x_{1}, \ldots, x_{n} ; t_{1}+h, \ldots, t_{n}+h\right)
$$


Na prática, essa condição é quase impossível de ser verificada, por isso são necessárias condições menos restritivas. Nesse sentido, costuma-se considerar condições da chamada de estacionariedade fraca.

Definição: 2.6 Um processo estocástico $\{X(t), t \in \tau\}$ é dito fracamente estacionário se, e somente se,

- $\mathbb{E}\{X(t)\}=\mu$, constante, para todo $t \in \tau$;

- $\mathbb{E}\left\{X(t)^{2}\right\}<\infty$, para todo $t \in \tau$;

- $\gamma\left(t_{1}, t_{2}\right)=\operatorname{Cov}\left\{X\left(t_{1}\right), X\left(t_{2}\right)\right\}$ é uma função apenas de $\left|t_{1}-t_{2}\right|$.

Definição 2.7: Um vetor real $p$-dimensional $\mathbf{X}=\left(X_{1}, \ldots, X_{p}\right)^{\prime}$ tem distribuição Normal multivariada de dimensão $p$, com vetor de médias $\mu=\left(\mu_{1}, \ldots, \mu_{p}\right)^{\prime}$ e matriz de covariâncias $\Sigma$ se sua densidade conjunta é dada por

$$
f_{\mathbf{x}}\left(x_{1}, \ldots, x_{p}\right)=(2 \pi)^{-\frac{p}{2}}|\Sigma|^{-\frac{1}{2}} \exp \left\{-\frac{1}{2}(\mathbf{x}-\mu)^{\prime} \Sigma^{-1}(\mathbf{x}-\mu)\right\},
$$

se $\boldsymbol{\Sigma}$ é não singular. Denota-se por: $\mathbf{X} \sim N_{p}(\mu, \boldsymbol{\Sigma})$.

- Se $\boldsymbol{\Sigma}$ é uma matriz diagonal, então os componentes do vetor $\mathbf{X}$ são independentes.

- $\mathrm{O} i$-ésimo componente de $\mathbf{X}$ possui distribuição normal univariada com média $\mu_{i}$ e variância $\sigma_{i}^{2}$.

Definição 2.8: Seja $\mathbf{X}=\mathbf{Y}+i \mathbf{Z}$ um vetor $p$-dimensional com componentes complexas, dizemos que $\mathbf{X}$ tem distribuição normal complexa multivariada com vetor de médias $\mu$ e matriz de covariâncias $\boldsymbol{\Sigma}$ se, e somente se, o vetor $(\mathbf{Y}, \mathbf{Z})^{\prime}$ de dimensão $2 p$ de componentes reais, tiver distribuição normal $2 p$-variada, com vetor de médias $(R e \mu, \operatorname{Im} \mu)^{\prime}$ e matriz de covariâncias $\mathbf{V}$, com

$$
\mathbf{V}=\frac{1}{2}\left[\begin{array}{cc}
\operatorname{Re} \boldsymbol{\Sigma} & -\operatorname{Im} \boldsymbol{\Sigma} \\
\operatorname{Im} \boldsymbol{\Sigma} & \operatorname{Re} \boldsymbol{\Sigma}
\end{array}\right]
$$

para algum vetor $\mu$ com dimensão $p$ e alguma matriz $\boldsymbol{\Sigma}$, de ordem $p \times p$, hermitiana e não negativa definida. Denota-se por: $\mathbf{X} \sim N_{p}^{c}(\mu, \boldsymbol{\Sigma})$.

- Seja X uma variável aleatória complexa de dimensão $p$, então

$$
\Sigma_{\mathbf{X X}}=\Sigma_{\mathbf{Y Y}}+\Sigma_{\mathbf{Z Z}}+i\left(\boldsymbol{\Sigma}_{\mathbf{Z Y}}-\boldsymbol{\Sigma}_{\mathbf{Y Z}}\right)
$$

- Se $X=Y+i Z$, então $\operatorname{Var}(X)=\operatorname{Var}(Y)+\operatorname{Var}(Z)$;

- Se $\mathbf{X} \sim N_{p}^{c}(\mu, \boldsymbol{\Sigma})$ e se $\operatorname{Im} \boldsymbol{\Sigma}=\mathbf{0}$, então as componentes de $\mathbf{X}$ são independentes.

\subsection{Propriedades da função de autocovariância}

Seja $\{X(t), t \in \mathbb{Z}\}$ um processo estocástico estacionário com tempo discreto. A função de autocovariância $\gamma(h)=\operatorname{Cov}\{X(t), X(t+h)\}$ satisfaz as seguintes propriedades:

- $\gamma(0)>0$

- $\gamma(h)=\gamma(-h)$, ou seja, a função de autocovariância é par; 
- $|\gamma(h)| \leq \gamma(0), \forall h \in \mathbb{Z}$;

- $\gamma(h)$ é não negativa definida, no sentido que

$$
\sum_{j=1}^{n} \sum_{k=1}^{n} a_{j} a_{k} \gamma\left(h_{j}-h_{k}\right) \geq 0,
$$

para quaisquer números reais $a_{1}, \ldots, a_{n}$ e $h_{1}, \ldots, h_{n}$ de $\mathbb{Z}$.

A função de autocorrelação de um processo estacionário é definida por

$$
\rho(h)=\frac{\gamma(h)}{\gamma(0)}, t \in \mathbb{Z},
$$

e possui as mesmas propriedades da função de autocovariância, sendo $\rho_{0}=1$.

\subsection{Transformações ortonormais}

As técnicas de análise de Fourier e de ondaletas nada mais são do que transformações de um processo estocástico. Suponha uma série temporal $\mathbf{X}=\left(X_{0}, \ldots, X_{T-1}\right)$ e uma matriz ortonormal. Multiplicando a série temporal pela matriz de transformação ortonormal, podemos observar os dados em um novo domínio, diferente do tempo. Além disso, podemos reconstruir a série original por meio de sua transformação inversa.

Definição 2.11: Seja $\mathbf{X}=\left(X_{0}, \ldots, X_{T-1}\right)$ uma série temporal de $T$ variáveis reais. Seja $\mathrm{Y}$ um outro vetor $T$-dimensional, o produto interno entre $\mathbf{X}$ e $\mathbf{Y}$ é dado por:

$$
\langle\mathbf{X}, \mathbf{Y}\rangle=\mathbf{X}^{\prime} \mathbf{Y}=\sum_{j=0}^{T-1} X_{j} Y_{j}
$$

Definição 2.12: Seja $\mathbf{X}=\left(X_{0}, \ldots, X_{T-1}\right)$ uma série temporal de $T$ variáveis reais. A norma ao quadrado de $\mathbf{X}$, também chamada de energia da série temporal é dada por:

$$
\|\mathbf{X}\|^{2}=\langle\mathbf{X}, \mathbf{X}\rangle=\mathbf{X}^{\prime} \mathbf{X}=\sum_{j=0}^{T-1} X_{j}^{2} .
$$

Definição 2.13: Uma matriz $\mathcal{P}$, quadrada de ordem $T$, é chamada de ortonormal se $\mathcal{P}^{\prime} \mathcal{P}=\mathcal{I}_{T}$, em que $\mathcal{I}_{T}$ é a matriz identidade de ordem $T$.

Seja $\mathcal{P}_{\bullet j}$ o vetor da j-ésima coluna de $\mathcal{P}$, e $\mathcal{P}_{k}$ o vetor que contém a k-ésima linha de $\mathcal{P}$. Pela ortonormalidade de $\mathcal{P}$ tem-se que

$$
\left\langle\mathcal{P}_{\bullet j}, \mathcal{P}_{\bullet j^{\prime}}\right\rangle= \begin{cases}1, & \text { se } j=j^{\prime} \\ 0, & \text { se } j \neq j^{\prime}\end{cases}
$$

Pode-se decompor a série temporal em coeficientes por meio da transformação ortonormal $\mathcal{P X}$, de tal forma que

$$
\mathbf{P}=\mathcal{P} \mathbf{X}=\left[\begin{array}{c}
\mathcal{P}^{\prime}{ }{ }_{\bullet} \mathbf{X} \\
\mathcal{P}^{\prime}{ }_{1 \bullet} \mathbf{X} \\
\vdots \\
\mathcal{P}^{\prime}{ }_{T-1}, \mathbf{X}
\end{array}\right]=\left[\begin{array}{c}
\left\langle\mathcal{P}_{0 \bullet}, \mathbf{X}\right\rangle \\
\left\langle\mathcal{P}_{1 \bullet}, \mathbf{X}\right\rangle \\
\vdots \\
\left\langle\mathcal{P}_{T-1 \bullet}, \mathbf{X}\right\rangle
\end{array}\right]
$$


$\mathrm{O}$ vetor $\mathbf{P}$ de ordem $T$ contém os coeficientes da transformação de $\mathbf{X}$ com respeito à transformação $\mathcal{P}$. Como $\mathbf{P}=\mathcal{P} \mathbf{X}$, basta pré multiplicar o vetor de coeficientes da transformação por $\mathcal{P}^{\prime}$ para reconstruir a série temporal $\mathbf{X}$, ou seja,

$$
\mathbf{X}=\mathcal{P}^{\prime} \mathbf{P}
$$

Uma propriedade importante das transformações ortonormais é a preservação da energia. Dessa maneira, a energia contida nos coeficientes da transformação $\left(\|\mathbf{P}\|^{2}\right)$ é igual à energia da série temporal $\left(\|\mathbf{X}\|^{2}\right)$, isto é,

$$
\|\mathbf{P}\|^{2}=\|\mathcal{P} \mathbf{X}\|^{2}=(\mathcal{P} \mathbf{X})^{\prime}(\mathcal{P} \mathbf{X})=\mathbf{X}^{\prime} \mathcal{P}^{\prime} \mathcal{P} \mathbf{X}=\mathbf{X}^{\prime} \mathbf{X}=\|\mathbf{X}\|^{2}
$$

portanto, dizemos que $\mathbf{X}$ e $\mathbf{P}$ são entidades matemáticas equivalentes.

Outra característica interessante das transformações ortonormais é o teorema da projeção. Suponha que deseja-se aproximar a série temporal $\mathbf{X}$ usando uma combinação linear de um subconjunto das linhas da matriz $\mathcal{P}$, assim temos que

$$
\hat{\mathbf{X}}=\sum_{j=0}^{T^{\prime}-1} \beta_{j} \mathcal{P}_{j \bullet}
$$

para $T^{\prime}<T$ e algum conjunto de coeficientes $\left\{\beta_{j}\right\}$. Dizemos $\hat{\mathbf{X}}$ é uma projeção de $\mathbf{X}$ em relação ao subespaço gerado por $\left(\mathcal{P}_{0 \bullet}, \mathcal{P}_{1 \bullet}, \ldots, \mathcal{P}_{T^{\prime}-1 \bullet}\right)$.

A teoria de transformações ortonormais pode ser estendida aos números complexos, como na transformada de Fourier. Assim, a propriedade de ortonormalidade se torna $\mathcal{P}^{H} \mathcal{P}=\mathcal{I}_{T}$, em que $\mathcal{P}^{H}$ diz respeito à transposta Hermitiana, que por definição é a matriz transposta original $\mathcal{P}^{\prime}$ substituindo seus elementos pelos respectivos complexos conjugados. Uma matriz $\mathcal{P}$ que satisfaz $\mathcal{P}^{H} \mathcal{P}=\mathcal{I}_{T}$ é chamada de matriz unitária. 


\section{Capítulo 3}

\section{Análise clássica de Fourier}

A análise de Fourier clássica tem como objetivo aproximar uma função $f(t)$ por meio de combinações lineares de senos e cossenos com diferentes frequências, para mais detalhes ver (Morettin, 2014) e (Chui, 1992). A base de Fourier é formada pelo conjunto $\left\{w_{n}(t)=\right.$ $\left.e^{i n t}, n \in \mathbb{Z}\right\}$ de funções ortogonais com período $2 \pi$.

Toda função periódica, de período $2 \pi$, de quadrado integrável pode ser reescrita através de combinações lineares das funções $w_{n}(t)$, que compõem a base de Fourier. Há relação entre o sistema de exponenciais complexas com o sistema de senos e cossenos $\{\cos (n t), \operatorname{sen}(n t), n \in$ $\mathbb{Z}\}$, dada pela fórmula de Euller

$$
e^{i n t}=\cos (n t)+i \operatorname{sen}(n t) .
$$

Definição 3.1: Uma função $f(t), t \in \mathbb{R}$, é periódica, de período $p$, se para todo $t \in \mathbb{R}$,

$$
f(t)=f(t+k p)
$$

para $k \in \mathbb{Z}$.

Sob suposições citadas anteriormente, uma função $f(t), t \in \mathbb{R}$, pode ser reescrita da seguinte maneira

$$
f(t)=\sum_{\lambda}\{a(\lambda) \cos (\lambda t)+b(\lambda) \operatorname{sen}(\lambda t)\}
$$

em que a representação acima pode ser uma soma finita ou infinita, ou até uma integral. Essa maneira de reescrever $f(t)$ é chamada de representação espectral de $f(t)$.

Basicamente, existem quatro situações: tempo contínuo e frequência contínua, tempo contínuo e frequência discreta, tempo discreto e frequência contínua, tempo discreto e frequência discreta. Nesse trabalho, analisaremos apenas o ultimo caso, tempo discreto e frequência discreta.

Seja $f_{0}, f_{1}, \ldots, f_{T-1}, T$ valores amostrados da função $f(t)$, a transformada discreta de Fourier da sequência $f_{j}, j=0, \ldots, T-1$ é dada por

$$
F_{n}=\sum_{j=0}^{T-1} f_{j} e^{-i \lambda_{n} j},
$$

em que $\lambda_{n}=\frac{2 \pi n}{T}, n=0, \ldots, T-1$ são chamadas frequências de Fourier. Pode-se mostrar 
que a transformada inversa é

$$
f_{t}=\frac{1}{T} \sum_{n=0}^{T-1} F_{n} e^{i \lambda_{n} t}
$$

Pela ortonormalidade da transformada de Fourier, segundo teorema de Parseval (Percival e Walden, 2000), o valor da soma quadrática de uma função periódica $f(t)$ com período $T$, é igual a média dos valores quadráticos dos coeficientes de Fourier, ou seja,

$$
\sum_{t=0}^{T-1}\left|f_{t}\right|^{2}=\frac{1}{T} \sum_{n=0}^{T-1}\left|F_{n}\right|^{2}
$$

esse teorema mostra que não há perda de informação quando transformamos os dados do domínio do tempo para o domínio da frequência e vice-versa.

\subsection{Análise Espectral de processos estacionários}

Considere um processo estocástico estacionário, $\{X(t), t \in \mathbb{Z}\}$, com média zero. Pelo fato de ser estacionário, segue-se que, se considerarmos qualquer trajetória desse processo teremos que ela não é de quadrado integrável e portanto não podemos definir a sua transformada de Fourier. Além disso, essa trajetória não é periódica, portanto não podemos considerar sua série de Fourier (Morettin, 2014).

Considere uma realização particular do processo, chamaremos de $X_{t}, t=0, \ldots, T-1$, agora $X_{t}$ não é mais uma função aleatória de $t$, definindo a função

$$
Y_{t}=\left\{\begin{array}{ccc}
X_{t}, & \text { se } t=0, \ldots, T-1 \\
0, & \text { se } t=T, T+1, \ldots
\end{array}\right.
$$

Para essa função, podemos definir a transformada de Fourier

$$
F(\lambda)=\sum_{t=0}^{\infty} Y(t) e^{-i \lambda t}=\sum_{t=0}^{T-1} X(t) e^{-i \lambda t},-\infty<\lambda<\infty .
$$

Temos que $|F(\lambda)|^{2}$ representa a energia total das componentes de $Y_{t}$ em torno de uma frequência $\lambda$. Segue que

$$
J(\lambda)=\frac{|F(\lambda)|^{2}}{T}
$$

representa a função de densidade de potência de $Y_{t}$, de modo que

$$
\lim _{T \rightarrow \infty} \frac{|F(\lambda)|^{2}}{T}
$$

descreve as propriedades espectrais de $X(t), t=0, \ldots, T-1$.

O limite acima depende de uma realização particular do processo, de modo que, para obter as propriedades espectrais do processo $\{X(t), t \in \mathbb{Z}\}$ devemos considerar

$$
f(\lambda)=\lim _{T \rightarrow \infty} E\{J(\lambda)\},
$$

se o limite existir, denomina-se $f(\lambda)$ de densidade espectral ou espectro do processo $\{X(t), t \in$ 
$\mathbb{Z}$ \}, em que o limite acima é igual à

$$
f(\lambda)=\frac{1}{2 \pi} \sum_{h=-\infty}^{\infty} \gamma_{h} e^{-i \lambda h}, \quad-\infty<\lambda<\infty,
$$

em que , $\gamma_{h}$ é a função de autocovariância do processo $\{X(t), t \in \mathbb{Z}\}$ (Morettin, 2014). Uma condição suficiente para esse limite existir é:

$$
\sum_{h=-\infty}^{\infty}\left|\gamma_{h}\right|<\infty
$$

A transformada inversa é dada por

$$
\gamma(h)=\int_{-\infty}^{\infty} f(\lambda) e^{i \lambda h} d \lambda
$$

note que

$$
\gamma(0)=\operatorname{Var}\{X(t)\}=\int_{-\infty}^{\infty} f(\lambda) d \lambda .
$$

Dessa maneira, a variância do processo pode ser interpretada como a "soma"de contribuições devidas às diversas componentes de frequências presente em $X(t)$.

Sob ponto de vista de informação probabilística, o espectro e a função de autocovariância são equivalentes.

Teorema 3.1: O espectro $f(\lambda)$ é limitado, não negativo, uniformemente contínuo, par e periódico de período $2 \pi$ (Morettin, 2014).

Pelo fato do especto $f(\lambda)$ ser periódico de período $2 \pi$, basta tomar uma parte de comprimento $2 \pi$ de seu domínio, por exemplo, $[-\pi, \pi]$, e como também é uma função par, basta visualizar apenas o intervalo $[0, \pi]$.

\subsection{Transformada discreta de Fourier e Periodograma}

Nessa sessão, trataremos da transformada Fourier finita, que é uma ferramenta estatística fundamental na análise espectral de séries temporais. Também introduziremos um estimador natural do espectro, chamado Periodograma.

Seja $\{X(t), t \in \mathbb{Z}\}$ um processo estacionário, a transformada de Fourier finita é definida por

$$
d_{\lambda}^{(T)}=\frac{1}{\sqrt{2 \pi T}} \sum_{t=0}^{T-1} X(t) e^{-i \lambda t}, \quad-\infty<\lambda<\infty .
$$

Como $d_{\lambda}^{(T)}=d_{\lambda+2 \pi}^{(T)}$, ou seja, periódico de período $2 \pi$, e $\overline{d_{-\lambda}^{(T)}}=d_{\lambda}^{(T)}$, portanto, par, basta considerar $\lambda \in[0, \pi]$.

Na prática, calculamos a transformada para um número finito de frequências, essas são da forma $\lambda_{n}=\frac{2 \pi n}{T}, \operatorname{com} \frac{(T-1)}{2} \leq n \leq \frac{T}{2}$, e são chamadas frequências de Fourier.

Teorema 3.2: Se o espectro $f(\lambda)$ for contínuo, então as variáveis aleatórias $d_{\lambda_{n}}^{(T)},-\left[\frac{T-1}{2}\right] \leq$ $n \leq \frac{T}{2}$, são assintoticamente independentes quando $T \rightarrow \infty$, com distribuição assintótica normal complexa $N_{1}^{c}\left(0, f\left(\lambda_{n}\right)\right)$ para $\lambda_{n} \neq \pi$ e $\lambda_{n} \neq 0$, e com distribuição assintótica normal $N_{1}\left(0, f\left(\lambda_{n}\right)\right)$ se $\lambda_{n}=\pi$ ou $\lambda_{n}=0$ (Brillinger, 1981).

Portanto, pelo teorema acima podemos afirmar que: 
(i) $E\left\{d_{\lambda_{n}}^{(T)}\right\}=0$;

(ii) $E\left\{\left|d_{\lambda_{n}}^{(T)}\right|^{2}\right\} \rightarrow f\left(\lambda_{n}\right)$ quando $T \rightarrow \infty$;

(iii) $E\left\{d_{\lambda_{n}}^{(T)} \overline{d_{\lambda_{m}}^{(T)}}\right\} \rightarrow 0$ quando $T \rightarrow \infty$ e $n \neq m$

Pelo fato de $E\left\{\left|d_{\lambda_{n}}^{(T)}\right|^{2}\right\} \approx f\left(\lambda_{n}\right)$, um estimador intuitivo para $f(\lambda)$ é o próprio quadrado do módulo do coeficiente da transformada discreta de Fourier. Esse estimador é chamado de Periodograma, definido por

$$
I_{\lambda_{n}}^{(T)}=\left|d_{\lambda_{n}}^{(T)}\right|^{2}=\frac{1}{2 \pi T}\left|\sum_{t=0}^{T-1} X(t) e^{-i \lambda_{n} t}\right|^{2},
$$

para as frequências $\lambda_{n}=\frac{2 \pi n}{T},\left[\frac{-(T-1)}{2}\right] \leq n \leq \frac{T}{2}$.

Como $E\left\{I_{\lambda_{n}}^{(T)}\right\}=E\left\{\left|d_{\lambda_{n}}^{(T)}\right|^{2}\right\} \rightarrow f\left(\lambda_{n}\right)$ quando $T \rightarrow \infty$, logo o Periodograma é um estimador assintoticamente não enviesado.

Teorema: 3.3 As variáveis aleatórias $I_{\lambda_{n}},-\left[\frac{T-1}{2}\right] \leq n \leq \frac{T}{2}$ são assintoticamente independentes e com distribuição assintótica múltipla de uma distribuição qui-quadrado com dois graus de liberdade $\chi_{(2)}^{2}$, se $\lambda_{n} \neq 0$ e $\lambda_{n} \neq \pi$ e com um grau de liberdade $\chi_{(1)}^{2}$, se $\lambda_{n}=0$ ou $\lambda_{n}=\pi$ (Brillinger, 1981).

Dessa maneira, assintoticamente

$$
\begin{aligned}
& \frac{2 I_{\lambda_{n}}}{f\left(\lambda_{n}\right)} \stackrel{d}{\longrightarrow} \chi_{(2)}^{2}, \text {, se } \lambda_{n} \neq 0 \text { e } \lambda_{n} \neq \pi, \\
& \frac{2 I_{\lambda_{n}}}{f\left(\lambda_{n}\right)} \stackrel{d}{\longrightarrow} \chi_{(1)}^{2}, \text {, se } \lambda_{n}=0 \text { ou } \lambda_{n}=\pi .
\end{aligned}
$$

Embora o periodograma seja assintoticamente não enviesado, ele não é consistente, pois a sua variância se mantém constante independente do tamanho da série. Para diminuir a variabilidade do periodograma a medida que o tamanho da amostra aumenta, uma alternativa é construir estimadores do espectro suavizados.

Podemos realizar suavizações no domínio do tempo e depois fazer a transformada de Fourier. Nesses casos, obtemos os chamados estimadores suavizados de covariância. Uma alternativa interessante é realizar a suavização no domínio da frequência, assim temos os chamados estimadores suavizados de periodograma.

O espectro de um processo estacionário é definido como

$$
f(\lambda)=\frac{1}{2 \pi} \sum_{h=-\infty}^{\infty} \gamma(h) e^{-i \lambda h}, \quad-\pi<\lambda<\pi .
$$

Na prática, não conhecemos $\gamma(h)$, então, precisamos substituir pelo seu estimador natural.

Definição 3.2: Seja $X_{0}, \ldots, X_{T-1}$ uma realização de um processo estocástico estacionário. A função de autocovariância amostral é definida por:

$$
\hat{\gamma}(h)= \begin{cases}\frac{1}{T} \sum_{t=0}^{T-1-|h|}\left\{X_{t}-\bar{X}\right\}\left\{X_{t+|h|}-\bar{X}\right\} & , \text { se }|h| \leq T-1 \\ 0 & \text {, se }|h|>T-1 .\end{cases}
$$


Sem perda de generalidade, supondo $E\{X(t)\}=0$, temos que

$$
\begin{aligned}
E\{\hat{\gamma}(h)\} & =\frac{1}{T} \sum_{t=0}^{T-1-|h|} E\left\{X_{t} X_{t+|h|}\right\}=\frac{1}{T} \sum_{t=0}^{T-1-|h|} \gamma(h) \\
& =\left\{\frac{T-|h|}{T}\right\} \gamma(h)=\left\{1-\frac{|h|}{T}\right\} \gamma(h) .
\end{aligned}
$$

Se a média do processo não for igual a zero, o viés do estimador é acrescido do termo $O\left(T^{-1}\right)$.

Temos que,

$$
E\left\{I^{(T)}(\lambda)\right\}=\frac{1}{2 \pi} \sum_{h=-T+1}^{T-1}\left(1-\frac{|h|}{T}\right) \gamma(h) e^{-i \lambda h},
$$

é a transformada de Fourier de $w(h) \gamma(h)$, em que

$$
w(h)=\left\{\begin{array}{cl}
1-\frac{|h|}{T} & , \text { se }|h| \leq T-1 \\
0 & , \text { se }|h|>T-1
\end{array}\right.
$$

Assim, tem-se que

$$
E\left\{I^{(T)}(\lambda)\right\}=\int_{-\pi}^{\pi} W(\alpha) f(\lambda-\alpha) d \alpha
$$

na qual $W(\lambda)$ é a transformada de Fourier de $w(h)$, dada por

$$
W(\lambda)=T\left[\frac{\operatorname{sen}(\pi T \lambda)}{\pi T \lambda}\right]^{2} .
$$

Uma função como $W(\lambda)$ é chamada de janela espectral. Para $T$ grande, a função $W(\lambda)$ se comporta como uma função delta de Dirac, implicando que $I^{(T)}(\lambda)$ é assintoticamente não enviesado.

Uma sequência $\left\{w_{M}(h), h \in \mathbb{Z}\right\}$, é um núcleo (ponderação) de comprimento $2 M$ se satisfaz as seguintes propriedades:

- $0 \leq w_{M}(h) \leq w_{M}(0)=1$;

- $w_{M}(h)=w_{M}(-h), \forall h$;

- $w_{M}(h)=0,|h|>M$.

Um estimador que leva em conta um núcleo $w_{M}(h)$,

$$
\hat{f}(\lambda)=\frac{1}{2 \pi} \sum_{h=-\infty}^{\infty} w(h) \hat{\gamma}_{h} e^{-i \lambda h}, \quad-\pi<\lambda<\pi,
$$

é chamado de estimador suavizado de covariâncias. Além disso, $W_{M}(\lambda)$ é uma janela espectral e satisfaz:

- $W(\lambda)=W_{M}(-\lambda), \forall \lambda$

- $\int_{-\pi}^{\pi} W_{M}(\lambda) d \lambda=w_{M}(0)=1$. 
O estimador $\hat{f}(\lambda)$ pode ser escrito como

$$
\hat{f}(\lambda)=\int_{-\pi}^{\pi} W_{M}(\lambda-\alpha) I^{(T)}(\alpha) d \alpha
$$

Considere ao invés de integral, a soma:

$$
\hat{f}(\lambda)=\frac{2 \pi}{T} \sum_{n=-(T-1) / 2}^{T / 2} W_{M}\left(\lambda-\lambda_{n}\right) I_{\lambda_{n}}^{(T)},
$$

em que $\lambda_{n}$ são as frequências de Fourier. O estimador acima é assintoticamente equivalente a

$$
\tilde{f}(\lambda)=\sum_{n=-(T-1) / 2}^{T / 2} W\left(\lambda-\lambda_{n}\right) I_{\lambda_{n}}^{(T)} .
$$

A estatística $\tilde{f}(\lambda)$ é chamada de estimador suavizado de periodogramas.

Um caso particular desse estimador foi sugerido por Daniell, e é formado por médias do periodograma em torno de uma frequência de interesse, ou seja,

$$
\tilde{I}(\lambda)=\frac{1}{2 m+1} \sum_{j=-m}^{m} I^{(T)}\left(\lambda+\frac{2 \pi j}{T}\right),
$$

em que $0 \leq m \leq(T-1) / 2$.

Seja $\{\bar{X}(t), t \in \mathbb{Z}\}$ um processo estacionário e $\tilde{I}(\lambda)$ o estimador de Daniell, então

$$
\operatorname{Var}(\tilde{I}(\lambda))= \begin{cases}\frac{f(\lambda)^{2}}{2 m+1}+O\left(T^{-1}\right), & \text { se } \lambda \neq 0 \text { e } \lambda \neq \pi \\ \frac{f(\lambda)^{2}}{m}+O\left(T^{-1}\right), & \text { se } \lambda=0 \text { ou } \lambda=\pi\end{cases}
$$

e

$$
\operatorname{Cov}\{\tilde{I}(\lambda), \tilde{I}(\mu)\}=O\left(T^{-1}\right) .
$$

As quantidades abaixo possuem a seguinte distribuição assintótica:

$$
\begin{array}{ll}
\frac{(4 m+2) \tilde{I}\left(\lambda_{j}\right)}{f\left(\lambda_{j}\right)} \stackrel{d}{\longrightarrow} \chi_{(4 m+2)}^{2}, \quad j=-m, \ldots, m, \text { se } \lambda_{j} \neq \pi \text { e } \lambda_{j} \neq 0 \\
\frac{(2 m) \tilde{I}\left(\lambda_{j}\right)}{f\left(\lambda_{j}\right)} \stackrel{d}{\longrightarrow} \chi_{(2 m)}^{2}, \quad j=-m, \ldots, m, \text { se } \lambda_{j}=\pi \text { ou } \lambda_{j}=0 .
\end{array}
$$

\subsection{Análise de variância usando a transformada discreta de Fourier}

Nessa seção, abordaremos a análise de variância no domínio da frequência que nada mais é do que uma extensão da análise de variância clássica. Pela natureza da transformada de Fourier, essa ferramenta é mais eficiente em processos estacionários. Para um tratamento mais aprofundado dessa técnica ver (Chiann e Toloi, 1993).

Uma abordagem comum na análise de variância pode ser escrita como o seguinte modelo 
de regressão linear (Roy et al., 1971):

$$
\mathbf{Y}=\mathbf{Z \Theta X}+\epsilon
$$

em que,

- Y é uma matriz de dimensão $N \times T$ das séries temporais observadas, em que $N$ corresponde ao número de unidades amostrais e $T$ o número de observações por unidade amostral;

- Z é uma matriz com dimensão $N \times M$ de planejamento dentro dos indivíduos;

- X é uma matriz com dimensão $S \times T$ de planejamento entre os indivíduos;

- $\Theta$ é uma matriz com dimensão $M \times S$ de parâmetros;

- $\epsilon$ é uma matriz com dimensão $N \times T$ de erros aleatórios.

O modelo pode ser reescrito como

$$
Y_{\ell}(t)=\sum_{j=1}^{S} \sum_{i=1}^{M} Z_{\ell i} \Theta_{i j} X_{j t}+\epsilon_{\ell t}, \quad \ell=1, \ldots, N, \quad t=0, \ldots, T-1,
$$

em que, $Y_{\ell t}$ representa $t$-ésima observação da $\ell$-ésima unidade amostral.

Um dos principais objetivos é realizar inferências sobre os parâmetros da matriz $\Theta$. Com os dados no domínio do tempo, devido a correlação entre as observações de uma mesma série temporal, os testes de hipóteses se tornam uma tarefa difícil. Utilizando o domínio da frequência, por meio da transformada discreta de Fourier, podemos proceder com inferências estatísticas mais facilmente pelo fato dos coeficientes de Fourier serem assintoticamente não correlacionados.

Considere $Y_{\ell}(t), t=0,1, \ldots, T-1, \ell=1, \ldots, N$, séries temporais reais. A transformada discreta de Fourier das observações é dada por:

$$
d_{\ell}^{(T)}(k)=\sum_{t=0}^{T-1} Y_{\ell}(t) e^{-i \lambda_{k} t}
$$

em que, $\lambda_{k}=\frac{2 \pi k}{T}, k=0, \ldots, \frac{T-1}{2}, \ell=1, \ldots, N$.

Os coeficientes de Fourier $d_{\ell}^{(T)}(k)$ podem ser representados como

$$
d_{\ell}^{(T)}(k)=\alpha_{\ell}(k)+O_{a . s}(1),
$$

para todo $\ell$, em que $\alpha_{\ell}(1), \ldots, \alpha_{\ell}\left(\frac{T-1}{2}\right)$ são variáveis com distribuição $N^{c}\left(0, f_{\ell}\left(\lambda_{k}\right)\right)$ independentes e $O_{a . s}(1) \rightarrow 0$ quase certamente, quando $T \rightarrow \infty$.

Para obtermos estimativas mais consistentes do espectro, podemos utilizar estimadores suavizados. Uma escolha simples é utilizar o estimador proposto por Daniell

$$
\tilde{I}\left(\lambda_{k}\right)=\frac{1}{2 m+1} \sum_{j=-m}^{m} I^{(T)}\left(\lambda_{k}+\frac{2 \pi j}{T}\right)
$$

em que

$$
\frac{(4 m+2) \tilde{I}\left(\lambda_{j}\right)}{f\left(\lambda_{k}\right)} \stackrel{d}{\longrightarrow} \chi_{(4 m+2)}^{2}
$$


Deve haver um equilíbrio no valor de $m$ escolhido, não pode ser muito grande se comparado ao tamanho da série, pois produz maior viés e nem muito pequeno, para não causar instabilidade nas estimativas.

\subsubsection{Modelo com um sinal em comum}

Um caso de interesse na aplicação da análise de variância é verificar a presença de um sinal em comum, $\Omega(t)$, entre $N$ séries temporais. Um possível modelo nesse caso é

$$
Y_{\ell}(t)=\mu_{\ell}+\Omega(t)+\epsilon_{\ell}(t),
$$

para $\ell=1, \ldots, N$ e $t=0, \ldots, T-1$.

\section{Modelo com sinal $\Omega(t)$}

Suponha que estejamos interessados em verificar se um conjunto de séries temporais possuem ou não sinais em comum. Temos que

- $\mu_{\ell}$ : o nível da $\ell$-ésima unidade amostral;

- $\Omega(t)$ : um sinal comum;

- $\epsilon_{\ell}$ : séries estacionárias com média zero, função de autocovariância $\gamma_{\epsilon}(h)$ e espectro $f_{\epsilon}(\lambda)$.

Pelas suposições acima

$$
E\left\{Y_{\ell}(t)\right\}=\mu_{\ell}+\Omega(t)
$$

e

$$
\begin{aligned}
& \operatorname{Cov}\left\{Y_{\ell}(t), Y_{\ell}(t+h)\right\}=\operatorname{Cov}\left\{\mu_{\ell}+\Omega(t)+\epsilon_{\ell}(t), \mu_{\ell}+\Omega(t+h)+\epsilon_{\ell}(t+h)\right\}= \\
& \operatorname{Cov}\left\{\epsilon_{\ell}(t), \epsilon_{\ell}(t+h)\right\}=\gamma_{\epsilon}(h)
\end{aligned}
$$

Estamos interessados em testar a hipótese da não existência de sinal comum, ou seja

$$
H_{0}: \Omega(t)=0,
$$

A transformada de Fourier com um sinal em comum é

$$
d_{\ell}(k)=\theta(k)+\varepsilon_{\ell}(k),
$$

para $k=0, \ldots,(T-1) / 2$, e $\ell=1, \ldots, N$ com $\varepsilon_{\ell}(k)$ variáveis aleatórias independentes com distribuição $N^{c}\left(0, f_{\epsilon}\left(\lambda_{k}\right)\right)$.

Considerando o periodograma suavizado para algum $K \in\{1,2, \ldots,(T-1) / 2\}$, podemos definir as seguintes quantidades:

$$
\begin{gathered}
S Q_{\Omega}=N \sum_{k=1}^{K}\left|\bar{d}_{\bullet}(k)\right|^{2}, \\
S Q_{R}=\sum_{k=1}^{K} \sum_{\ell=1}^{N}\left|d_{\ell}(k)-\bar{d}_{\bullet}(k)\right|^{2},
\end{gathered}
$$


em que

$$
\bar{d}_{\bullet}(k)=\frac{1}{N} \sum_{\ell=1}^{N} d_{\ell}(k) .
$$

Sob a hipótese nula, as temos que as quantidades convergem

$$
\frac{2 S Q_{\Omega}}{f_{\epsilon}(\lambda)} \stackrel{d}{\longrightarrow} \chi_{2 K}^{2}
$$

e

$$
\frac{2 S Q_{R}}{f_{\epsilon}(\lambda)} \stackrel{d}{\longrightarrow} \chi_{2 K(N-1)}^{2} .
$$

Para testar a hipótese nula podemos usar a estatística

$$
F(\lambda)=\frac{\frac{2 S Q_{\Omega}(\lambda)}{2 K f_{\epsilon}(\lambda)}}{\frac{2 S Q_{R}(\lambda)}{2 K(N-1) f_{\epsilon}(\lambda)}}=\frac{(N-1) S Q_{\Omega}(\lambda)}{S Q_{R}(\lambda)} \stackrel{d}{\longrightarrow} F_{(2 K, 2 K(N-1))} .
$$

\begin{tabular}{|c|c|c|c|c|}
\hline $\begin{array}{l}\text { Fonte } \\
\text { de va- } \\
\text { riação }\end{array}$ & $\begin{array}{l}\text { Graus de liber- } \\
\text { dade }\end{array}$ & Soma de quadrados $(S Q)$ & Quadrado médio $(Q M)$ & $F$ \\
\hline$\Omega$ & $2 K$ & $N \sum_{k=1}^{K}\left|\bar{d}_{\bullet}(k)\right|^{2}$ & $S Q_{\Omega} / 2 K$ & $\frac{Q M_{\Omega}}{Q M_{R}}$ \\
\hline Resíduo & $2 K(N-1)$ & $\sum_{k=1}^{K} \sum_{\ell=1}^{N}\left|d_{\ell}(k)-\bar{d}_{\bullet}(k)\right|^{2}$ & $S Q_{R} / 2 K(N-1)$ & \\
\hline Total & $2 K N$ & $\sum_{k} \sum_{\ell=1}^{N}\left|d_{\ell}(k)\right|^{2}$ & & \\
\hline
\end{tabular}

Um resumo desse procedimento é dado pela Tabela ANOVA em 3.1.

Tabela 3.1: ANOVA para testar diferença de sinais em comum na frequência $\lambda_{k}$.

Sem a utilização de estimadores suavizados do espectro a estatística de teste $F$, fica

$$
F\left(\lambda_{k}\right)=\frac{(N-1)\left|\bar{d}_{k}\right|^{2}}{\sum_{n=1}^{N}\left|d_{n k}-\bar{d}_{k}\right|^{2}} \stackrel{d}{\longrightarrow} F_{(2,2(N-1))}
$$

para $\lambda_{k}=\frac{2 \pi k}{T}, k=0, \ldots,(T-1) / 2$.

\subsubsection{Modelo com um fator em comum}

Suponha agora que exista o interesse em verificar diferença entre $M$ tratamentos (níveis) no contexto de séries temporais. O modelo apropriado é

para $\ell=1, \ldots, L_{m}, m=1, \ldots, M, t=0, \ldots, T-1, N=L_{1}, \ldots, L_{M}$ em que,

- $L_{m}$ : número de unidades amostrais submetido ao $m$-ésimo tratamento;

- $\Gamma_{m}$ : o efeito do $m$-ésimo tratamento. 
Modelo com sinais $\Omega(t)$ e $\Gamma_{m}(t)$

Digamos que o pesquisador está interessado em verificar se existe diferença entre tratamentos previamente fixados em que,

- $\mu_{l m}$ : o nível da l-ésima unidade amostral dentro do $m$-ésimo tratamento;

- $\Omega(t)$ : o sinal comum entre todas as unidades amostrais;

- $\Gamma_{m}$ : o sinal (vetor de tamanho $T$ ) em comum entre todas as unidades amostrais do $m$-ésimo tratamento, é determinístico com a seguinte restrição para obter a identificabilidade:

$$
\sum_{m=1}^{M} L_{m} \Gamma_{m}(t)=0
$$

- $\epsilon_{\ell m}$ são séries estacionárias com média zero, função de autocovariância $\gamma_{\epsilon}(h)$ e espectro $f_{\epsilon}(\lambda)$.

Pelas suposições acima

$$
E\left\{Y_{\ell m}(t)\right\}=\mu_{\ell m}+\Omega(t)+\Gamma_{m}(t)
$$

e

$$
\operatorname{Cov}\left\{Y_{\ell m}(t), Y_{\ell m}(t+h)\right\}=\operatorname{Cov}\left\{\epsilon_{\ell m}(t), \epsilon_{\ell m}(t+h)\right\}=\gamma_{\epsilon}(h) .
$$

Testar a diferença entre os tratamentos, que equivale a testar

$$
H_{0}: \Gamma_{1}(t)=\Gamma_{2}(t)=\ldots=\Gamma_{M}(t)=0 .
$$

O modelo pode ser reescrito como

$$
d_{\ell m}(k)=\theta(k)+\tau_{m}(k)+\varepsilon_{\ell m}(k),
$$

para $\ell=1, \ldots, L_{m}, m=1, \ldots, M, k=0, \ldots, T-1$ com $\varepsilon_{\ell m}(k) \sim N^{c}\left(0, f_{\epsilon}\left(\lambda_{k}\right)\right)$ independentes.

Considerando o periodograma suavizado para algum $\mathrm{K} \in\{1,2, \ldots,(T-1) / 2\}$, podemos definir as seguintes quantidades:

$$
\begin{gathered}
S Q_{\Omega}=N \sum_{k=1}^{K}\left|\bar{d}_{\bullet \bullet}(k)\right|^{2}, \\
S Q_{\Gamma}=\sum_{k=1}^{K} \sum_{m=1}^{M} L_{m}\left|\bar{d}_{\bullet m}(k)-\bar{d}_{\bullet \bullet}(k)\right|^{2}, \\
S Q_{R}=\sum_{k=1}^{K} \sum_{m=1}^{M} \sum_{\ell=1}^{L_{m}}\left|d_{\ell m}(k)-\bar{d}_{\bullet m}(k)\right|^{2},
\end{gathered}
$$

em que $\bar{d}_{m}(k)=\frac{1}{L_{m}} \sum_{\ell=1}^{L_{m}}\left|d_{\ell m}(k)\right|^{2}$ e $\bar{d}(k)=\frac{1}{N} \sum_{m=1}^{M} \sum_{\ell=1}^{L_{m}}\left|d_{\ell m}(k)\right|^{2}$. 
Sob a hipótese de $\Gamma_{1}(t)=\Gamma_{2}(t)=\ldots=\Gamma_{M}(t)=0$,

$$
\frac{2 S Q_{\Gamma}}{f_{\epsilon}(\lambda)} \stackrel{d}{\longrightarrow} \cdot \chi_{2 K(N-1)}^{2}
$$

além disso,

$$
\frac{2 S Q_{R}}{f_{\epsilon}(\lambda)} \stackrel{d}{\longrightarrow} \cdot \chi_{2 K(N-M)}^{2},
$$

e as estatísticas acima são independentes.

Para verificar a existência de sinal em comum entre os tratamentos, podemos utilizar a estatística de teste

$$
F\left(\lambda_{k}\right)=\frac{\frac{2 S Q_{\Gamma}(\lambda)}{2 K(M-1) f_{\epsilon}(\lambda)}}{\frac{2 S Q_{R}(\lambda)}{2 K(N-M) f_{\epsilon}(\lambda)}}=\frac{(N-M) S Q_{\Gamma}(\lambda)}{(M-1) S Q_{R}(\lambda)} \stackrel{d}{\longrightarrow} F_{(2 K(M-1), 2 K(N-M))},
$$

para $\lambda_{k}=\frac{2 \pi k}{T}, k=0, \ldots, \frac{T-1}{2}$.

A Tabela ANOVA é dada em 3.2.

\begin{tabular}{|c|c|c|c|c|}
\hline $\begin{array}{l}\text { Fonte } \\
\text { de va- } \\
\text { riação }\end{array}$ & $\begin{array}{l}\text { Graus de liber- } \\
\text { dade }\end{array}$ & Soma de quadrados $(S Q)$ & Quadrado médio $(Q M)$ & $F$ \\
\hline$\Omega$ & $2 K$ & $N \sum_{k=1}^{K}\left|\bar{d}_{\bullet \bullet}(k)\right|^{2}$ & $S Q_{\Omega} / 2 K$ & $\frac{Q M_{\Omega}}{Q M_{R}}$ \\
\hline$\Gamma$ & $2 K(M-1)$ & $\sum_{k=1}^{K} \sum_{s=1}^{M} L_{m}\left|\bar{d}_{\bullet m}(k)-\bar{d}_{\bullet \bullet}(k)\right|^{2}$ & $S Q_{\Gamma} / 2 K(M-1)$ & $\frac{Q M_{\Gamma}}{Q M_{R}}$ \\
\hline Resíduo & $2 K(N-M)$ & $\sum_{k=1}^{K} \sum_{m=1}^{M} \sum_{\ell=1}^{L_{m}}\left|d_{\ell m s}(k)-\bar{d}_{\bullet m s}(k)\right|^{2}$ & $S Q_{R} / 2 K(N-M)$ & \\
\hline Total & $2 K N$ & $\sum_{k} \sum_{m=1}^{M} \sum_{\ell=1}^{L_{m}}\left|d_{\ell m s}(k)\right|^{2}$ & & \\
\hline
\end{tabular}

Tabela 3.2: ANOVA para testar diferença de sinais determinísticos de tratamentos na frequência $\lambda_{k}$.

Utilizando as componentes espectrais não suavizadas, temos a estatística para testar a existência de sinal em comum entre os tratamentos e entre as séries,

$$
F\left(\lambda_{k}\right)=\frac{(N-M) \sum_{m=1}^{M} L_{m}\left|\bar{d}_{\bullet \bullet}(k)-\bar{d}_{\bullet m}(k)\right|^{2}}{\sum_{m=1}^{M} \sum_{\ell=1}^{L_{m}}\left|d_{\ell m}(k)-\bar{d}_{\bullet m}(k)\right|^{2}} \stackrel{d}{\longrightarrow} F_{(2(M-1), 2(N-M))} .
$$

\subsubsection{Modelo com interação entre dois fatores}

Suponha agora que exista o interesse em verificar a interação entre dois grupos de tratamentos, o primeiro grupo com $M$ tratamentos (níveis) e segundo grupo com $S$ tratamentos (níveis). No contexto de séries temporais, o modelo apropriado é

$$
Y_{\ell m s}(t)=\mu_{\ell m s}+\Omega(t)+\Gamma_{m}(t)+\Theta_{s}(t)+\Lambda_{m s}(t)+\epsilon_{\ell m s}(t),
$$

para $\ell=1, \ldots, L_{m s}, m=1, \ldots, M, s=1, \ldots, S, t=0, \ldots, T-1$, em que,

- $L_{m s}$ : número de unidades amostrais submetido ao $m$-ésimo tratamento do primeiro grupo e ao $s$-ésimo tratamento do segundo grupo;

- $\Gamma_{m}$ : o efeito do $m$-ésimo tratamento do primeiro grupo; 
- $\Theta_{s}$ : o efeito do s-ésimo tratamento do segundo grupo;

- $\Lambda_{m s}$ : o efeito de interação $m$-ésimo tratamento do primeiro grupo com o $s$-ésimo tratamento do segundo grupo.

Modelo com sinais $\Omega(t), \Gamma_{m}(t), \Theta_{s}(t)$ e $\Lambda_{m s}(t)$

Digamos que o pesquisador está interessado em verificar se existe efeito de interação entre dois grupos de tratamentos previamente fixados em que,

- $\mu_{l m s}$ : o nível da l-ésima unidade amostral dentro do m-ésimo tratamento;

- $\Omega(t)$ : o sinal comum entre todas as unidades amostrais;

- $\Gamma_{m}$ : o sinal (vetor de tamanho $T$ ) em comum entre todas as unidades amostrais do $m$-ésimo tratamento do primeiro grupo, é determinístico com a seguinte restrição para obter a identificabilidade:

$$
\sum_{s=1}^{S} \sum_{m=1}^{M} L_{m s} \Gamma_{m}(t)=0 ;
$$

- $\Theta_{s}$ : o sinal (vetor de tamanho $T$ ) em comum entre todas as unidades amostrais do $s$-ésimo tratamento do segundo grupo, é determinístico com a seguinte restrição para obter a identificabilidade:

$$
\sum_{s=1}^{S} \sum_{m=1}^{M} L_{m s} \Theta_{s}(t)=0 ;
$$

- $\Lambda_{m s}$ : o sinal (vetor de tamanho $T$ ) em comum entre todas as unidades amostrais do $m$-ésimo tratamento do primeiro grupo e $s$-ésimo tratamento do segundo grupo, é determinístico com a seguinte restrição para obter a identificabilidade:

$$
\sum_{s=1}^{S} \sum_{m=1}^{M} L_{m s} \Lambda_{m s}(t)=0
$$

- $\epsilon_{\ell m s}$ são séries estacionárias com média zero, função de autocovariância $\gamma_{\epsilon}(h)$ e espectro $f_{\epsilon}(\lambda)$.

Pelas suposições acima

$$
E\left\{Y_{\ell m}(t)\right\}=\mu_{\ell m s}+\Omega(t)+\Gamma_{m}(t)+\Theta_{s}(t)+\Lambda_{m s}(t)
$$

e

$$
\operatorname{Cov}\left\{Y_{\ell m}(t), Y_{\ell m}(t+h)\right\}=\operatorname{Cov}\left\{\epsilon_{\ell m}(t), \epsilon_{\ell m}(t+h)\right\}=\gamma_{\epsilon}(h) .
$$

Testar a inexistência de interação é equivalente a testar a seguinte hipótese:

$$
H_{0}: \Lambda_{11}(t)=\Lambda_{12}(t)=\ldots=\Lambda_{M S}(t)=0
$$


Aplicando a transformada de Fourier, o modelo 4.3.3 pode ser reescrito como

$$
d_{\ell m s}(k)=\theta(k)+\tau_{m}(k)+\eta_{s}(k)+\delta_{m s}(k)+\varepsilon_{\ell m s}(k),
$$

para $\ell=1, \ldots, L_{m s}, m=1, \ldots, M, s=1, \ldots, S, k=0, \ldots, T-1 \operatorname{com} \varepsilon_{\ell m s}(k) \sim N^{c}\left(0, f_{\epsilon}\left(\lambda_{k}\right)\right)$ independentes, com a restrição $\sum_{m=1}^{M} \sum_{s=1}^{S} L_{m s} \tau_{m}(k)=0, \sum_{m=1}^{M} \sum_{s=1}^{S} L_{m s} \eta_{s}(k)=0$ e $\sum_{m=1}^{M} \sum_{s=1}^{S} L_{m s} \delta_{m s}(k)=$ 0 para $k$ fixos.

Considerando o periodograma suavizado, para algum $\mathrm{K} \in\{1,2, \ldots,(T-1) / 2\}$, podemos definir as seguintes quantidades:

$$
\begin{gathered}
S Q_{\Omega}=N \sum_{k=1}^{K}\left|\bar{d}_{\bullet \bullet}(k)\right|^{2} \\
S Q_{\Gamma}=\sum_{k=1}^{K} \sum_{m=1}^{M} L_{m}\left|\bar{d}_{\bullet m \bullet}(k)-\bar{d}_{\bullet \bullet \bullet}(k)\right|^{2} \\
S Q_{\Theta}=\sum_{k=1}^{K} \sum_{s=1}^{S} L_{s}\left|\bar{d}_{\bullet \bullet}(k)-\bar{d}_{\bullet \bullet \bullet}(k)\right|^{2} \\
S Q_{\Lambda}=\sum_{k=1}^{K} \sum_{s=1}^{S} \sum_{m=1}^{M} L_{m s}\left|\bar{d}_{\bullet m s}(k)-\bar{d}_{\bullet m \bullet}(k)-\bar{d}_{\bullet \bullet s}(k)+\bar{d}_{\bullet \bullet \bullet}(k)\right|^{2}, \\
S Q_{R}=\sum_{k=1}^{K} \sum_{m=1}^{M} \sum_{s=1}^{S} \sum_{\ell=1}^{L_{m s}}\left|d_{\ell m s}(k)-\bar{d}_{\bullet m s}(k)\right|^{2}
\end{gathered}
$$

em que $\bar{d}_{\bullet m \bullet}(k)=\frac{1}{L_{m}} \sum_{\ell=1}^{L_{m}}\left|d_{\ell m s}(k)\right|^{2}, \bar{d}_{\bullet \bullet}(k)=\frac{1}{L_{s}} \sum_{\ell=1}^{L_{s}}\left|d_{\ell m s}(k)\right|^{2}$ e $\bar{d}_{\bullet \bullet \bullet}(k)=\frac{1}{N} \sum_{s=1}^{S} \sum_{m=1}^{M} \sum_{\ell=1}^{L_{m s}}\left|d_{\ell m s}(k)\right|^{2}$.

Sob a hipótese de $\Lambda_{11}(t)=\Lambda_{12}(t)=\ldots=\Lambda_{M S}(t)=0$,

$$
\frac{2 S Q_{\Lambda}}{f_{\epsilon}(\lambda)} \stackrel{d}{\longrightarrow} \chi_{2 K(M-1)(S-1)}^{2} .
$$

Além disso,

$$
\frac{2 S Q_{R}}{f_{\epsilon}(\lambda)} \stackrel{d}{\longrightarrow} \cdot \chi_{2 K(N-M S)}^{2} .
$$

E as estatísticas acima são independentes.

Para verificar a existência de sinal em comum entre os tratamentos, podemos utilizar a estatística de teste

$$
F\left(\lambda_{k}\right)=\frac{\frac{2 S Q_{\Lambda}(\lambda)}{2 K(M-1)(S-1) f_{\epsilon}(\lambda)}}{\frac{2 S Q_{R}(\lambda)}{2 K(N-M) f_{\epsilon}(\lambda)}}=\frac{(N-M S) S Q_{\Lambda}(\lambda)}{(M-1)(S-1) S Q_{R}(\lambda)} \stackrel{d}{\longrightarrow} F_{(2 K(M-1)(S-1), 2 K(N-M S))},
$$

para $\lambda_{k}=\frac{2 \pi k}{T}, k=0, \ldots, \frac{T-1}{2}$.

Caso não seja rejeitada a hipótese testada acima, podemos testar o efeito de cada um dos grupos de tratamento separadamente.

A Tabela ANOVA é dada em 3.3.

Utilizando as componentes espectrais não suavizadas, temos a seguinte estatística de 
Tabela 3.3: ANOVA para testar sinal determinístico de interação entre grupos de tratamentos na frequência $\lambda_{k}$.

\begin{tabular}{|c|c|c|c|c|}
\hline $\begin{array}{l}\text { Fonte } \\
\text { de va- } \\
\text { riação }\end{array}$ & $\begin{array}{l}\text { Graus de liber- } \\
\text { dade }\end{array}$ & Soma de quadrados $(S Q)$ & Quadrado médio $(Q M)$ & $F$ \\
\hline$\Omega$ & $2 K$ & $N \sum_{k=1}^{K}\left|\bar{d}_{\bullet \bullet \bullet}(k)\right|^{2}$ & $S Q_{\Omega} / 2 K$ & $\frac{Q M_{\Omega}}{Q M_{R}}$ \\
\hline$\Gamma$ & $2 K(M-1)$ & $\sum_{k=1}^{K} \sum_{s=1}^{M} L_{m}\left|\bar{d}_{\bullet m \bullet}(k)-\bar{d}_{\bullet \bullet \bullet}(k)\right|^{2}$ & $S Q_{\Gamma} / 2 K(M-1)$ & $\frac{Q M_{\Gamma}}{Q M_{R}}$ \\
\hline$\Theta$ & $2 K(S-1)$ & $\sum_{k=1}^{K} \sum_{s=1}^{S} L_{s}\left|\bar{d}_{\bullet \bullet s}(k)-\bar{d}_{\bullet \bullet \bullet}(k)\right|^{2}$ & $S Q_{\Theta} / 2 K(S-1)$ & $\frac{Q M_{\Theta}}{Q M_{R}}$ \\
\hline$\Lambda$ & $2 K(M-1)(S-1)$ & $\sum_{k=1}^{K} \sum_{s=1}^{S} \sum_{m=1}^{M} L_{m s} \mid \bar{d}_{\bullet} m s(k)-\bar{d}_{\bullet m} \bullet$ & $S Q_{\Lambda} / 2 K(M-1)(S-1)$ & $\frac{Q M_{\Lambda}}{Q M_{R}}$ \\
\hline Resíduo & $2 K(N-M S)$ & $\sum_{k=1}^{K} \sum_{m=1}^{M} \sum_{s=1}^{S} \sum_{\ell=1}^{L_{m s}}\left|d_{\ell m s}(k)-\bar{d}_{\bullet m s}(k)\right|^{2}$ & $S Q_{R} / 2 K(N-M S)$ & \\
\hline Total & $2 K N$ & $\sum_{k} \sum_{m=1}^{M} \sum_{s=1}^{S} \sum_{\ell=1}^{L_{m s}}\left|d_{\ell m s}(k)\right|^{2}$ & & \\
\hline
\end{tabular}

teste para verificar a hipótese existência de interação:

$$
F\left(\lambda_{k}\right)=\frac{(N-M S) \sum_{s=1}^{S} \sum_{m=1}^{M} L_{m s}\left|\bar{d}_{\bullet m s}(k)-\bar{d}_{\bullet m \bullet}(k)-\bar{d}_{\bullet \bullet s}(k)+\bar{d}_{\bullet \bullet \bullet}(k)\right|^{2}}{(M-1)(S-1) \sum_{k=1}^{K} \sum_{m=1}^{M} \sum_{s=1}^{S} \sum_{\ell=1}^{L_{m s}}\left|d_{\ell m s}(k)-\bar{d}_{\bullet m s}(k)\right|^{2}} \stackrel{d}{\longrightarrow} F_{((M-1)(S-1),(N-M S))},
$$

para $\lambda_{k}=\frac{2 \pi k}{T}, k=0, \ldots, \frac{T-1}{2}$. 


\section{Capítulo 4}

\section{Análise de ondaletas}

As ondaletas (wavelets) são pequenas ondas que crescem e depois decaem para zero em um curto intervalo. As ondaletas podem ser vistas como diferentes bases para representar funções além da conhecida base de Fourier. No caso de Fourier, consideramos funções que pertencem a $L^{2}(0,2 \pi)$, agora queremos considerar o espaço $L^{2}(\mathbb{R})$ de funções mensuráveis definidas na reta real, que satisfazem

$$
\int_{-\infty}^{\infty}|f(x)|^{2} d x<\infty .
$$

Toda função em $L^{2}(\mathbb{R})$ deve decair para zero quando $x \rightarrow \pm \infty$, e isso não ocorre com as funções seno e cosseno. Portanto, estamos interessado em funções com esse tipo de comportamento, as chamadas ondaletas são funções que tem essa propriedade e podem gerar o $L^{2}(\mathbb{R})$.

De maneira análoga à Fourier, a ideia é ter uma única função $\psi$ que gere todo o espaço de funções. Para cobrir toda reta, são utilizadas translações da função $\psi$. Além disso, trabalhamos com diferentes "frequências". Assim, consideramos funções da forma

$$
\psi_{a, b}(x)=|a|^{\frac{-1}{2}} \psi\left(\frac{x-b}{a}\right), b \in \mathbb{R}, a \in \mathbb{R},
$$

observe que $\psi_{a, b}(x)$ é obtida através de translações e dilatações da função $\psi(x)$, o termo $|a|^{\frac{-1}{2}}$ que multiplica a função é uma constante normalizadora, ou seja, usada para que a norma de $\psi_{a, b}(x)$ seja igual a 1 .

Por conveniência de notação e questões computacionais $a$ e $b$ tomam os valores: $a=2^{-j}$ e $b=k 2^{-j}$. Nesse cenário obtemos a seguinte função

$$
\psi_{j, k}(x)=2^{\frac{j}{2}} \psi\left(2^{j} x-k\right), j, k \in \mathbb{Z},
$$

portanto, as funções $\psi_{j, k}(x)$ são obtidas por meio de translações diádicas $k 2^{-j}$ e dilatações binárias $2^{-j}$. As funções $\left\{\psi_{j, k}(x), j, k \in \mathbb{Z}\right\}$ não formam necessariamente uma base ortogonal.

Considere uma base ortogonal

$$
\left\{\psi_{j, k}(x), j, k \in \mathbb{Z}\right\}
$$

Logo,

$$
\left\langle\psi_{j, k}, \psi_{l, m}\right\rangle=\int_{-\infty}^{\infty} \psi_{j, k}(x) \psi_{l, m}(x) d x=\delta_{j l} \delta_{k m}, \quad j, k, l, m \in \mathbb{Z}
$$


em que,

$$
\delta_{j l}= \begin{cases}1, & j=l \\ 0, & j \neq l\end{cases}
$$

Assim como na análise de Fourier, podemos representar uma função $f(x)$ por meio de combinação linear. A $f(x)$ pode ser representada por uma séries de ondaletas através da combinação linear

$$
f(x)=\sum_{j=-\infty}^{\infty} \sum_{k=-\infty}^{\infty} c_{j, k} \psi_{j, k}(x),
$$

em que os coeficientes de ondaletas podem ser obtidos por:

$$
c_{j, k}=\left\langle f, \psi_{j, k}\right\rangle=\int_{-\infty}^{\infty} f(x) \psi_{j, k}(x) d x .
$$

O teorema de Parseval também é válido no caso das séries de ondaletas,

$$
\int_{-\infty}^{\infty} f(x)^{2} d x=\sum_{j=-\infty}^{\infty} \sum_{k=-\infty}^{\infty} c_{j, k}^{2} .
$$

A função $\psi(x)$ é denominada ondaleta mãe e satisfaz algumas propriedades como,

i $\quad \int_{-\infty}^{\infty} \psi(x) d x=0$

ii $\int_{-\infty}^{\infty}|\psi(x)| d x<\infty$;

iii $\int_{-\infty}^{\infty} \frac{|\Psi(\omega)|}{\omega} d \omega=C<\infty$, em que $\Psi(\omega)$ é a transformada de Fourier de $\psi(x)$.

A terceira propriedade é chamada de condição de admissibilidade. Essa condição garante a reconstrução da função $f(x)$ após a aplicação da transformada de ondaletas.

A seguir são exibidos alguns exemplos de ondaletas mãe.
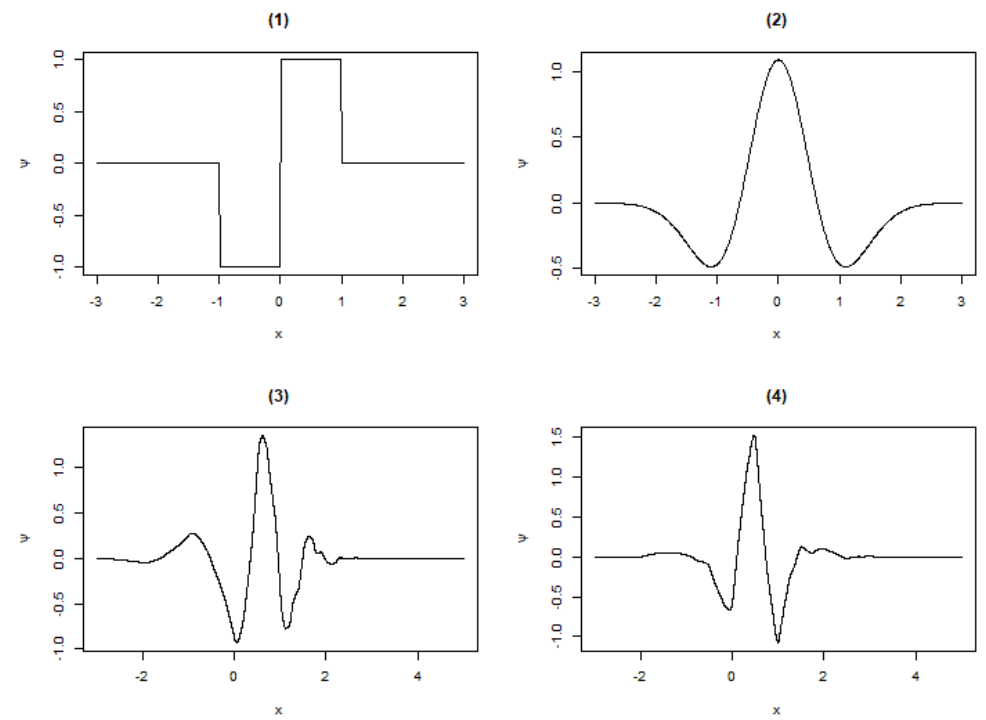

Figura 4.1: (1) Haar; (2) Mexican Hat; (3) Extremal Phase D4; (4) Least Asymmetric LA4. 
Uma forma de gerar ondaleta mãe $\psi$ é pela função escala, chamada também de ondaleta pai, denotada por $\phi$. Uma ondaleta pai $\phi$ é obtida pela solução da equação

$$
\phi(x)=2^{\frac{1}{2}} \sum_{k} l_{k} \phi(2 x-k) .
$$

Depois de obtida a ondaleta pai, podemos definir funções a partir de translações e dilatações de $\phi$ :

$$
\phi_{j, k}(x)=2^{\frac{j}{2}} \phi\left(2^{j} x-k\right), \quad j, k \in \mathbb{Z} .
$$

As ondaletas mães, então, podem ser obtidas por meio da seguinte relação

$$
\psi(x)=2^{\frac{1}{2}} \sum_{k} h_{k} \phi(2 x-k),
$$

em que $l_{k}$ é um filtro passa baixo (low pass) e $h_{k}$ um filtro passa alto (high pass), a relação entre esses dois filtros dada por: $h_{k}=(-1)^{k} l_{1-k}$ (Morettin, 2014).

A função $\phi$ forma uma família ortogonal e gera uma análise de multirresolução em $L^{2}(\mathbb{R})$.

\subsection{Análise de Multirresolução}

A Análise de Multirresolução é uma ferramenta que nos permite dar um "zoom"em uma função, possibilitando analisá-la em diferentes níveis de resolução. Formalmente, suponha uma sequência de subespaços fechados $\left\{V_{j}, j \in \mathbb{Z}\right\}$, com as seguintes propriedades

$$
\begin{array}{ll}
\text { i } & \cdots \subset V_{-1} \subset V_{0} \subset V_{1} \subset \ldots ; \\
\text { ii } & \bigcup_{j \in \mathbb{Z}} V_{j}=L^{2}(\mathbb{R}) ; \\
\text { iii } \bigcap_{j \in \mathbb{Z}} V_{j}=\{0\} ; \\
\text { iv } V_{j+1}=V_{j} \oplus W_{j}, \quad V_{j} \perp W_{j}, j \in \mathbb{Z} ; \\
\text { v } f(x) \in V_{j} \Leftrightarrow f(2 x) \in V_{j+1}, j \in \mathbb{Z} .
\end{array}
$$

A família de funções $\left\{\phi_{j, k}, k \in \mathbb{Z}\right\}$ forma uma base em $V_{j}$.

Quando passamos do subespaço $V_{j}$ para o subespaço $V_{j+1}$ ganhamos informação sobre a função, de modo que podemos aproximar $f$ o quanto desejarmos, fazendo com que $j$ (nível de resolução) cresça. Por outro lado, quando passamos do subespaço $V_{j}$ para o subespaço $V_{j+1}$ perdemos informação sobre a $f$. Em cada subespaço $V_{j}$, a melhor aproximação de uma função $f \in L^{2}(\mathbb{R})$ é a projeção ortogonal da $f$ em $V_{j}$.

Pela propriedade iv podemos reescrever $V_{j}$ como,

$$
V_{j}=V_{l} \oplus \bigoplus_{m=l}^{j-1} W_{m}=\bigoplus_{m=-\infty}^{j-1} W_{m}
$$

Temos que as ondaletas $\left\{\psi_{j, k}, k \in \mathbb{Z}\right\}$, geradas pela ondaleta pai $\phi$, formam uma base ortogonal para $W_{j}$. Pelo fato da $\bigcup_{j=-\infty}^{\infty} W_{j}$ ser denso em $L^{2}(\mathbb{R})$, o sistema $\left\{\psi_{j, k}, j, k \in \mathbb{Z}\right\}$ forma uma base ortogonal para $L^{2}(\mathbb{R})$. 
Como podemos aproximar $f$ o quanto desejarmos, então existe $J$ tal que $f_{J} \in V_{J}$ aproxima $f$. Seja $g_{j} \in W_{j}$, tem-se que

$$
f_{J}=f_{J-1}+g_{J-1}
$$

Recursivamente,

$$
f_{J}=g_{J-1}+g_{J-2}+\ldots+g_{J-M}+f_{J-M} .
$$

Qualquer $f \in L^{2}(\mathbb{R})$ pode ser reescrita como,

$$
f(x)=\sum_{k} c_{j_{0}, k} \phi_{j_{0}, k}+\sum_{j \geq j_{0}} \sum_{k} d_{j, k} \psi_{j, k}(x),
$$

em que,

$$
\begin{aligned}
c_{j_{0}, k} & =\int_{-\infty}^{\infty} f(x) \phi_{j_{0}, k}(x) d x, \\
d_{j, k} & =\int_{-\infty}^{\infty} f(x) \psi_{j, k}(x) d x,
\end{aligned}
$$

e $j_{0}$ é o nível de resolução mais baixo.

\subsection{Transformada discreta de ondaletas}

Assim como no caso de Fourier, existe também a transformada discreta de ondaletas (TDO), que é uma ferramenta também aplicada na análise de séries temporais, e possui propriedades interessantes para dados não estacionários. A transformada discreta de ondaletas é definida por:

$$
\begin{gathered}
w_{j k}=\sum_{t=0}^{T-1} X(t) \psi_{j k}(t), j=j_{0}, j_{0}+1, \ldots, J, k=0, \ldots, 2^{j}-1, \\
v_{j_{0} k}=\sum_{t=0}^{T-1} X(t) \phi_{j_{0} k}(t), k=0, \ldots, 2^{j_{0}}-1,
\end{gathered}
$$

em que $j$ é o nível de resolução, $k$ é o parâmetro de translação da ondaleta e está relacionado com o tempo, $J$ é o maior nível de resolução, $j_{0}$ é o menor nível de resolução. Os coeficientes $w_{j k}$ são os responsáveis pelos detalhes da série, principalmente em níveis de resolução mais altos. Os coeficientes $v_{j_{0} k}$ são os principais responsáveis pela tendência da série. Em muitos casos, define-se $j_{0}=0$, porém não necessariamente este é o valor ótimo a se considerar para o nível de resolução mais baixo (Abramovich, 2000).

Considere $\mathbf{W}=\left(w_{J 0}, w_{J 1}, \ldots, w_{J_{0} 0}, v_{J_{0} 0}\right)^{\prime}$ o vetor que contém os coeficientes da transformada de ondaletas. Em termos matriciais a transformada pode ser representada como: 


$$
\mathbf{W}=\left(\begin{array}{c}
w_{J, 0} \\
w_{J, 1} \\
\vdots \\
w_{J, 2^{J}-1} \\
w_{J-1,0} \\
\vdots \\
w_{J_{0} 0} \\
v_{J_{0} 0}
\end{array}\right)=\left(\begin{array}{c}
\sum_{t} X(t) \psi_{J, 0}(t) \\
\sum_{t} X(t) \psi_{J, 1}(t) \\
\vdots \\
\sum_{t} X(t) \psi_{J, 2^{J}-1}(t) \\
\sum_{t} X(t) \psi_{J-1,0}(t) \\
\vdots \\
\sum_{t} X(t) \psi_{J_{0}, 0}(t) \\
\sum_{t} X(t) \phi_{J_{0}, 0}(t)
\end{array}\right)=\mathcal{W X}
$$

em que $\mathcal{W}$ é a matriz formada por translações de dilatações do vetor $\psi_{\mathbf{j k}}=\left(\psi_{j k}(0), \psi_{j k}(1), \ldots\right.$, $\left.\psi_{j k}(T-1)\right)^{\prime}$ da primeira até a penúltima linha e $\phi_{\mathbf{j} \mathbf{k}}=\left(\phi_{j_{0} k}(0), \phi_{j_{0} k}(1), \ldots, \phi_{j_{0} k}(T-1)\right)^{\prime}$ na sua última linha. Na prática, não é realizado o produto matricial para obter os coeficientes da TDO. Os coeficientes são encontrados por meio de um algoritmo eficiente (Mallat, 1989) de complexidade $O(N)$, o qual é mais rápido que a própria transformada rápida de Fourier que possui complexidade $O(N \log N)$.

Assim como a TDF, a TDO possui uma interpretação física, uma vez que cada elemento do vetor $\mathbf{W}$ está associado a uma escala e conjunto de tempos específicos. Uma diferença relevante entre a TDO e a TDF é que os coeficientes de ondaletas são localizados no tempo e na escala, por isso trazem informação local da série, enquanto os coeficientes de Fourier são localizados na frequência, o que dá a esses uma característica global sobre a informação da série. Dessa maneira, a TDO se mostra uma ferramenta interessante, especialmente quando a série temporal não apresenta estacionariedade.

Para ilustrar a transformada discreta de ondaletas, vamos começar pela primeira ondaleta que se tem registro, a ondaleta de Haar, datada de 1910. Suponha uma série temporal $\mathbf{X}=\left(X_{0}, X_{1}, \ldots, X_{7}\right)$. A ondaleta mãe de Haar é dada por

$$
\psi(t)= \begin{cases}-1, & \text { se } 0 \leq t<1 / 2 \\ +1, & \text { se } 1 / 2 \leq t<1\end{cases}
$$

A matriz da transformação tomando $J=2$ e $J_{0}=0$ é

$$
\mathcal{W}=\left(\begin{array}{l}
\psi_{\mathbf{2 0}}{ }^{\prime} \\
\psi_{\mathbf{2 1}}{ }^{\prime} \\
\psi_{\mathbf{2 2}}{ }^{\prime} \\
\psi_{\mathbf{2 3}}{ }^{\prime} \\
\psi_{\mathbf{1 0}}{ }^{\prime} \\
\psi_{\mathbf{1 1}}{ }^{\prime} \\
\psi_{\mathbf{0 0}}{ }^{\prime} \\
\phi_{\mathbf{0 0}}{ }^{\prime}
\end{array}\right)=\left(\begin{array}{cccccccc}
-1 / \sqrt{2} & 1 / \sqrt{2} & 0 & 0 & 0 & 0 & 0 & 0 \\
0 & 0 & -1 / \sqrt{2} & 1 / \sqrt{2} & 0 & 0 & 0 & 0 \\
0 & 0 & 0 & 0 & -1 / \sqrt{2} & 1 / \sqrt{2} & 0 & 0 \\
0 & 0 & 0 & 0 & 0 & 0 & -1 / \sqrt{2} & 1 / \sqrt{2} \\
-1 / 2 & -1 / 2 & 1 / 2 & 1 / 2 & 0 & 0 & 0 & 0 \\
0 & 0 & 0 & 0 & -1 / 2 & -1 / 2 & 1 / 2 & 1 / 2 \\
-1 / \sqrt{8} & -1 / \sqrt{8} & -1 / \sqrt{8} & -1 / \sqrt{8} & 1 / \sqrt{8} & 1 / \sqrt{8} & 1 / \sqrt{8} & 1 / \sqrt{8} \\
1 / \sqrt{8} & 1 / \sqrt{8} & 1 / \sqrt{8} & 1 / \sqrt{8} & 1 / \sqrt{8} & 1 / \sqrt{8} & 1 / \sqrt{8} & 1 / \sqrt{8}
\end{array}\right) .
$$

Realizando o produto $\mathcal{W} \mathbf{X}$ é obtido o vetor $\mathbf{W}=\left(w_{20}, w_{21}, \ldots, w_{00}, v_{00}\right)$. Os $T / 2$ primeiros coeficientes de ondaletas, nesse caso $w_{20}, w_{21}, w_{22}$ e $w_{21}$, estão relacionados com o nível mais fino, $j=2$, dos detalhes. Os próximos $T / 4$ coeficientes, $w_{10}$ e $w_{11}$ estão relacionados com nível $j=1$ dos detalhes, o coeficiente de ondaletas $w_{00}$ está associado ao nível $j=0$ dos detalhes, e o coeficiente $v_{00}$ está associado à média dos valores de $\mathbf{X}$. Esse último coeficiente 
recebe o nome de coeficiente de escala.

Suponha uma série temporal $\mathbf{X}$ tenha tamanho $T=2^{J+1}$, e seja $\mathbf{W}$ o vetor de coeficientes da TDO. Podemos particionar o vetor $\mathbf{W}$ da seguinte maneira

$$
\mathbf{W}=\left[\begin{array}{c}
\mathbf{W}_{J} \\
\mathbf{W}_{J-1} \\
\vdots \\
\mathbf{W}_{j_{0}} \\
\mathbf{V}_{j_{0}}
\end{array}\right]
$$

em que, $\mathbf{W}_{J}$ é o subvetor que contém os coeficientes de ondaletas do nível $j=J, \mathbf{W}_{J-1}$ é o subvetor que contém os coeficientes de ondaletas do nível $j=J-1$, e assim por diante. Além disso, $\mathbf{V}_{j_{0}}$ contém os coeficientes de escala.

Com essa partição, pode-se observar a energia da série $\mathbf{X}$ em diferentes escalas

$$
\|\mathbf{X}\|^{2}=\|\mathbf{W}\|^{2}=\sum_{j=j_{0}}^{J}\left\|\mathbf{W}_{j}\right\|^{2}+\left\|\mathbf{V}_{j_{0}}\right\|^{2},
$$

em que $\left\|\mathbf{W}_{j}\right\|^{2}$ representa a quantidade de energia da série devido à escala $j$. A decomposição da variância nos permite construir o escalograma na escala $2^{j}\left\{S_{\mathcal{W}}(j), j=0,1, \ldots, J\right\}$ de $\mathbf{X}$ como

$$
S_{\mathcal{W}}(j)=\frac{1}{T}\left\|\mathbf{W}_{\mathbf{j}}\right\|^{2}=\frac{1}{T} \sum_{k} w_{j k}^{2} .
$$

Podemos analisar a síntese da série $\mathbf{X}$ por meio das diferentes escalas realizando a transformada inversa,

$$
\mathbf{X}=\mathcal{W}^{\prime} \mathbf{W}=\sum_{j=j_{0}}^{J} \mathcal{W}_{j}^{\prime} \mathbf{W}_{j}+\mathcal{V}_{j_{0}} \mathbf{V}_{j_{0}}
$$

em que, $\mathcal{W}_{j}$ e $\mathcal{V}_{j_{0}}$ são partições da matriz de transformação $\mathcal{W}$. Dessa maneira, podemos definir $\mathbf{D}_{j} \equiv \mathcal{W}_{j} \mathbf{W}_{j}$ para $j=0, \ldots, J$, o vetor $T$ dimensional de elementos associados com mudanças de $\mathbf{X}$ na escala $2^{j}$. Definindo também $\mathbf{S}_{j_{0}}=\mathcal{V}_{j_{0}} \mathbf{V}_{j_{0}}$ o vetor o vetor $T$ dimensional responsável pela escala, podemos reconstruir totalmente a série temporal como,

$$
\mathbf{X}=\sum_{j=j_{0}}^{J} \mathbf{D}_{j}+\mathbf{S}_{j_{0}}
$$

\subsubsection{Propriedades probabilísticas da transformada discreta de on- daletas}

Sob certas suposições, as propriedades sobre a distribuição da TDO são conhecidas. Primeiramente, a respeito a estacionariedade da série temporal, seja $\{X(t), t \in \mathbb{Z}\}$ um processo fracamente estacionário de média zero, e seja ainda $\gamma(h)=E\{X(t) X(t+h)\}, h \in \mathbb{Z}$, a função de autocovariância de $X(t)$. Além disso, iremos supor que,

$$
\sum_{h=-\infty}^{\infty}(1+|h|)|\gamma(h)|<\infty .
$$


Se as suposições são satisfeitas então o espectro de ondaletas do processo $\{X(t), t \in \mathbb{Z}\}$ em $(j, k)$ com respeito à ondaleta $\psi$, é definido por

$$
\eta_{j, k}=\sum_{h=-\infty}^{\infty} \gamma(h) \Psi_{j, k}(h)
$$

em que,

$$
\Psi_{j, k}(h)=\sum_{t=0}^{\infty} \psi_{j, k}(t) \psi_{j, k}(t+|h|)
$$

é chamada de função de autocovariância de ondaletas no ponto $(j, k)$.

Seja $X_{0}, X_{1}, \ldots, X_{T-1}$ uma série temporal de $T=2^{J+1}$. Para $j=1,2, \ldots, J$ e $k=$ $0,1, \ldots, 2^{j}-1$, tem-se que os coeficientes da transformada discreta de ondaletas possuem as seguintes propriedades

$$
\begin{gathered}
E\left\{w_{j, k}\right\}=0, \\
\operatorname{Var}\left\{w_{j, k}\right\}=\sum_{h=-(T-1)}^{T-1} \gamma(h) \sum_{t=0}^{T-1-|h|} \psi_{j, k}(t) \psi_{j, k}(t+|h|),
\end{gathered}
$$

é possível perceber que $\operatorname{Var}\left\{w_{j, k}\right\} \rightarrow \eta_{j, k}$ quando $T \rightarrow \infty$.

Teorema 4.1: (Chiann e Morettin, 1998) Suponha que as suposições anteriores estejam satisfeitas, e seja

$$
\eta_{\left(j, j^{\prime}, k, k^{\prime}\right)}=\sum_{h=-\infty}^{\infty} \gamma(h) \sum_{t=0}^{\infty} \psi_{j, k}(t+|h| I(h>0)) \psi_{j^{\prime}, k^{\prime}}(t+|h| I(h<0)),
$$

para $j, j^{\prime}, k, k^{\prime} \in \mathbb{Z}$. Então,

i $E\left\{w_{j, k} w_{j^{\prime}, k^{\prime}}\right\} \rightarrow \eta_{\left(j, j^{\prime}, k, k^{\prime}\right)}$ quando $T \rightarrow \infty$

ii Se $j=j^{\prime}$ e $k=k^{\prime}$, então $\eta_{\left(j, j^{\prime}, k, k^{\prime}\right)}=\eta_{(j, k)}$.

A quantidade $\eta_{\left(j, j^{\prime}, k, k^{\prime}\right)}$ é chamada de covariância assintótica da TDO. A covariância entre pares de coeficientes da TDO não é necessariamente igual a 0, mas em algumas situações podemos ter independência.

Teorema 4.2: (Chiann e Morettin, 1998) Seja $X(t), t \in\{0, \ldots, T-1\}$ um processo estritamente estacionário, $\mathbf{W}$ o vetor aleatório da transformada de ondaletas e suponha que $E\left\{w_{j, k} w_{j^{\prime}, k^{\prime}}\right\} \rightarrow \eta_{\left(j, j^{\prime}, k, k^{\prime}\right)}$ quando $T \rightarrow \infty$, então $\mathbf{W}$ converge em distribuição para uma normal multivariada com vetor de médias nulo e matriz de covariâncias no qual os elementos são dados por $\eta_{\left(j, j^{\prime}, k, k^{\prime}\right)}$.

Em particular, para $j$ e $k$ quaisquer,

$$
w_{j, k} \stackrel{d}{\longrightarrow} N\left(0, \eta_{(j, k)}\right) .
$$

\subsubsection{Transformada discreta de ondaletas não decimada}

Na literatura são encontrados diferentes nomes para transformada discreta de ondaletas não decimada (TDOND), entre elas transformada estacionária e transformada invariante no 
tempo. Na prática, a ondaleta não decimada é obtida fazendo $a=2^{-j}$ e $b=k$ em 4.2:

$$
\psi_{j, k}(x)=2^{\frac{j}{2}} \psi\left(2^{j}(x-k)\right), j, k \in \mathbb{Z},
$$

A transformada discreta de ondaletas não decimada é uma modificação de transformada discreta de ondaletas (TDO). Ela possui esse nome, pois diferentemente da TDO, não ocorre a decimação por 2 no número de coeficientes a cada nível da transformada. A TDOND é redundante, pois realiza translações mínimas $k$ em cada uma das escala, diferente da TDO em que as translações $2^{-j} k$ dependem da escala, consequentemente o número de coeficientes da TDOND é o mesmo em todas as escalas.

Diferente da TDO, a TDOND é uma transformação não ortogonal, portanto a inversa da matriz de transformação não é dada pela transposta da matriz de transformação como no caso da TDO. De fato, a matriz de transformação $\mathcal{W}$ da série temporal $X_{0}, X_{1}, \ldots, X_{T-1}$ tem dimensão $\left(J-j_{0}+2\right) T \times T$. Com isso, é necessário fazer um redimensionamento da matriz de transformação para que, após a aplicação da TDOND, possamos realizar a transformação inversa sem perda da informação.

Para reconstruir a série temporal $\mathbf{X}$, primeiramente, devemos pós-multiplicar a transposta da matriz de transformação $\mathcal{W}^{\prime}$ por uma matriz diagonal de pesos $\mathbf{T}$ definida por

$$
\mathbf{T}=\operatorname{diag}(\overbrace{1 / 2^{j_{0}}, \ldots, 1 / 2^{j_{0}}}^{2 T}, \ldots, \overbrace{1 / 2^{J-1}, \ldots, 1 / 2^{J-1}}^{T}, \overbrace{1 / 2^{J}, \ldots, 1 / 2^{J}}^{T}) .
$$

As colunas da matriz $\mathbf{V}=\mathbf{T}^{1 / 2} \mathcal{W}$ formam um conjunto ortonormal de modo que

$$
\mathbf{V}^{\prime} \mathbf{V}=I_{T}
$$

Então, a reconstrução de $\mathbf{X}$ a partir dos coeficientes $\mathbf{W}$ da TDOND é realizada por meio da seguinte operação

$$
\mathbf{X}=\mathcal{W}^{\prime} \mathbf{T W} .
$$

Um problema que surge na TDOND é a usual presença de autocorrelação entre os coeficientes da transformada de um mesmo nível $j$, fato que não ocorre na TDO, em que os coeficientes são não correlacionados assintoticamente.

A autocorrelação compromete a aplicação da Análise de variância (ANOVA), porém existem métodos que provocam efeito de quase descorrelação dos coeficientes(Soltani et al., 2004). A ideia é aplicar uma transformação nos coeficientes originais dividindo cada nível de resolução $j$ em dois grupos e depois calcular a média dos quadrados dos respectivos pares, da seguinte maneira,

$$
W_{j k}=\frac{w_{j k}^{2}+w_{j k+T / 2}^{2}}{2}, j=j_{0}, j_{0}+1, \ldots, J, k=0,1, \ldots,(T-1) / 2 .
$$

Essa transformação diminui o efeito da autocorrelação presente nos coeficientes da TDOND, além disso, $\log _{2} W_{j k}$ é assintoticamente normal com média $-(2 H+1) j+C$, onde $H$ é o expoente de Hurst e $C$ é uma constante que independe do nível de resolução $j$.

A seguir é apresentada as estimativas da a função de autocorrelação da TDOND depois e antes da aplicação da transformação 4.40 em uma série que será utilizada no capitulo 6 . 

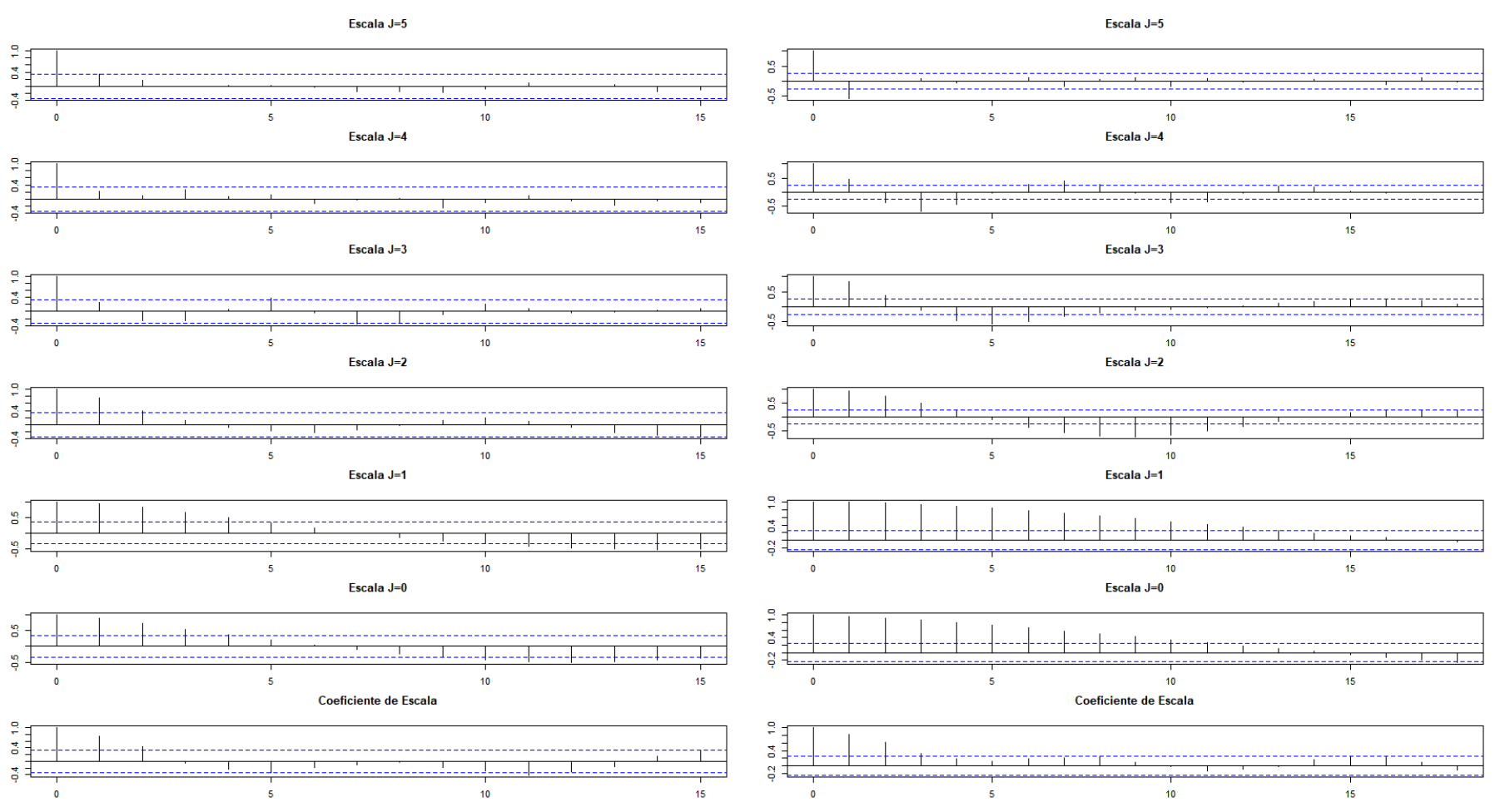

Figura 4.2: Função de autocorrelação típica da TDOND após e antes da aplicação da transformação 4.40 .

\subsection{Análise de variância usando a transformada discreta de ondaletas}

\subsubsection{Modelo com sinal em comum}

Suponha que para um determinado instante de tempo $t \in\{0, \ldots, T-1\}$ as observações sejam modeladas por um modelo de análise de variância do tipo.

$$
Y_{\ell}(t)=\mu_{\ell}+\Omega(t)+\epsilon_{\ell}(t)
$$

para $\ell=1, \ldots, N$ e $t=0, \ldots, T-1$.

Modelo com sinal $\Omega(t)$

Suponha que estejamos interessados em verificar se um conjunto de séries temporais possuem ou não sinais em comum. Temos que

- $\mu_{\ell}$ : o nível da $\ell$-ésima unidade amostral;

- $\Omega(t)$ : um sinal comum;

- $\epsilon_{\ell}$ são séries estacionárias com média zero.

Estamos interessados em testar a hipótese da existência de sinal comum, ou seja

$$
H_{0}: \Omega(t)=0
$$


Seja $\mathbf{W}=\mathcal{W} \mathbf{y}$ a transformada de ondaletas de $\mathbf{y}$. Nesse caso os índices de tempo são substituídos pelos índices de tempo e escala $(j, k)$ da análise de multirresolução. A transformada de ondaletas com um sinal em comum é

$$
w_{\ell}(j, k)=\mu_{\ell}+\theta(j, k)+\varepsilon_{\ell}(j, k),
$$

em que, $\varepsilon_{\ell}(j, k) \sim N\left(0, \sigma^{2}\right)$ para $j$ e $k$ fixos.

Podemos definir as seguintes soma dos quadrados:

$$
\begin{gathered}
S Q_{\Omega}=N \bar{w}_{\bullet}(j, k)^{2} ; \\
S Q_{R}=\sum_{\ell=1}^{N}\left(w_{\ell}(j, k)-\bar{w}_{\bullet}(j, k)\right)^{2},
\end{gathered}
$$

em que

$$
\bar{w}_{\bullet}(j, k)=\frac{1}{N} w_{\ell}(j, k)
$$

Sob a hipótese nula, temos que as quantidades convergem (Montgomery, 2017) e (Pinheiro e Vidakov 1993).

$$
\frac{S Q_{\Omega}}{\sigma^{2}} \stackrel{d}{\longrightarrow} \chi_{1}^{2}
$$

$\mathrm{e}$

$$
\frac{S Q_{R}}{\sigma^{2}} \stackrel{d}{\longrightarrow} \chi_{(N-1)}^{2}
$$

\begin{tabular}{|c|c|c|c|c|}
\hline $\begin{array}{l}\text { Fonte } \\
\text { de va- } \\
\text { riação }\end{array}$ & $\begin{array}{l}\text { Graus de liber- } \\
\text { dade }\end{array}$ & Soma de quadrados $(S Q)$ & Quadrado médio $(Q M)$ & $F$ \\
\hline$\Omega$ & 1 & $N \bar{w}_{\bullet}(j, k)^{2}$ & $S Q_{\Omega}$ & $\frac{Q M_{\Omega}}{Q M_{R}}$ \\
\hline Resíduo & $(N-1)$ & $\sum_{\ell=1}^{N}\left(w_{\ell}(j, k)-\bar{w}_{\bullet}(j, k)\right)^{2}$ & $S Q_{R} /(N-1)$ & \\
\hline Total & $N$ & $\sum_{\ell=1}^{N}\left|w_{\ell}(j, k)\right|^{2}$ & & \\
\hline
\end{tabular}

Para testar a hipótese nula podemos usar a estatística

$$
F(j, k)=\frac{\frac{S Q_{\Omega}}{\sigma^{2}}}{\frac{S Q_{R}(j, k)}{(N-1) \sigma^{2}}}=\frac{(N-1) S Q_{\Omega}(j, k)}{S Q_{R}} \stackrel{d}{\longrightarrow} F_{(1,(N-1))} .
$$

Um resumo desse procedimento é dado pela Tabela ANOVA em 4.1

Tabela 4.1: ANOVA para testar diferença de sinais em comum para índices $(j, k)$.

\subsubsection{Modelo com um fator em comum}

Suponha que para um determinado instante de tempo $t \in\{0, \ldots, T-1\}$ as observações sejam modeladas por um modelo de análise de variância do tipo.

$$
Y_{\ell m}(t)=\mu_{\ell}+\Omega(t)+\Gamma_{m}(t)+\epsilon_{\ell m}(t),
$$


para $\ell=1, \ldots, L_{m}, m=1, \ldots, M$ e $t=0, \ldots, T-1$,

Modelo com sinais $\Omega(t)$ e $\Gamma_{m}(t)$

Digamos que o pesquisador está interessado em verificar se existe diferença entre tratamentos previamente fixados em que,

- $\mu_{l m}$ : o nível da l-ésima unidade amostral dentro do $m$-ésimo tratamento;

- $\Omega$ : o sinal comum entre todas as unidades amostrais;

- $\Gamma_{m}$ : o sinal (vetor de tamanho $T$ ) em comum entre todas as unidades amostrais do $m$-ésimo tratamento, é determinístico com a seguinte restrição para obter a identificabilidade:

$$
\sum_{m=1}^{M} L_{m} \Gamma_{m}(t)=0
$$

- $\epsilon_{\ell m}$ são séries estacionárias com média zero.

Aplicando a transformada de ondaletas em 4.50,

$$
w_{\ell m}(j, k)=\theta(j, k)+\tau_{m}(j, k)+\varepsilon_{\ell m}(j, k),
$$

com a restrição $\sum_{m=1}^{M} \tau_{m}(j, k)=0$ para $j$ e $k$ fixos.

Para testar

$$
H_{0}: \tau_{1}(j, k)=\tau_{2}(j, k)=\ldots=\tau_{M}(j, k)=0 .
$$

Definindo as somas dos quadrados:

$$
\begin{aligned}
S Q_{\Gamma} & =\sum_{m=1}^{M} L_{m}\left(\bar{w}_{\bullet m}(j, k)-\bar{w}_{\bullet \bullet}(j, k)\right)^{2} \\
S Q_{R} & =\sum_{m=1}^{M} \sum_{\ell=1}^{L_{m}}\left(w_{\ell m}(j, k)-\bar{w}_{\bullet m}(j, k)\right)^{2}
\end{aligned}
$$

em que, $L_{m}$ é o número de unidades amostrais no $m$-ésimo nível, $\bar{w}_{\bullet \bullet}(j, k)=\frac{1}{N} \sum_{m=1}^{M} \sum_{\ell=1}^{L_{m}} w_{\ell m}$ é a média geral, $\bar{w}_{\bullet m}(j, k)=\frac{1}{L_{m}} \sum_{\ell=1}^{L_{m}} w_{\ell m}$ é a média no $m$-ésimo nível. 1993),

Sob $H_{0}$ as seguintes quantidades convergem (Montgomery, 2017) e (Pinheiro e Vidakovic,

$$
\begin{aligned}
& \frac{S Q_{\Omega}}{\sigma^{2}} \stackrel{d}{\longrightarrow} \chi_{M-1}^{2}, \\
& \frac{S Q_{R}}{\sigma^{2}} \stackrel{d}{\longrightarrow} \chi_{N-M}^{2},
\end{aligned}
$$

em que, $N=\sum_{m=1}^{M} L_{m}$ 
Para verificar a existência de sinal em comum entre os tratamentos, podemos utilizar a seguinte estatística de teste para cada $j$ e $k$,

$$
F(j, k)=\frac{(N-M) S Q_{\Omega}}{(M-1) S Q_{R}} \stackrel{d}{\longrightarrow} F_{M-1, N-M}
$$

Um resumo desse procedimento é dado pela Tabela ANOVA em 4.2.

Tabela 4.2: ANOVA para testar diferença de sinais determinísticos de tratamentos nos índices $(j, k)$.

\begin{tabular}{|c|c|c|c|c|}
\hline $\begin{array}{l}\text { Fonte } \\
\text { de va- } \\
\text { riação }\end{array}$ & $\begin{array}{l}\text { Graus de liber- } \\
\text { dade }\end{array}$ & Soma de quadrados $(S Q)$ & Quadrado médio $(Q M)$ & $F$ \\
\hline$\Omega$ & 1 & $N\left(\bar{w}_{\bullet \bullet}(j, k)\right)^{2}$ & $S Q_{\Omega}$ & $\frac{Q M_{\Omega}}{Q M_{R}}$ \\
\hline$\Gamma$ & $(M-1)$ & $\sum_{m=1}^{M} L_{m}\left(\bar{w}_{\bullet m}(j, k)-\bar{w}_{\bullet \bullet}(j, k)\right)^{2}$ & $S Q_{\Gamma} /(M-1)$ & $\frac{Q M_{\Gamma}}{Q M_{R}}$ \\
\hline Resíduo & $(N-M)$ & $\sum_{m=1}^{M} \sum_{\ell=1}^{L_{m}}\left(w_{\ell m}(j, k)-\bar{w}_{\bullet m}(j, k)\right)^{2}$ & $S Q_{R} /(N-M)$ & \\
\hline Total & $N$ & $\sum_{m=1}^{M} \sum_{\ell=1}^{L_{m}}\left(w_{\ell m}(j, k)\right)^{2}$ & & \\
\hline
\end{tabular}

\subsubsection{Modelo com interação entre dois fatores}

Suponha que para um determinado instante de tempo $t \in\{0, \ldots, N-1\}$ as observações sejam modeladas por um modelo de análise de variância do tipo.

$$
Y_{\ell m s}(t)=\mu_{\ell m s}+\Omega(t)+\Gamma_{m}(t)+\Theta_{s}(t)+\Lambda_{m s}(t)+\epsilon_{\ell m s}(t),
$$

para $\ell=1, \ldots, L_{m s}, m=1, \ldots, M, s=1, \ldots, S, t=0, \ldots, T-1, N=L_{11}$, em que,

- $L_{m s}$ : número de unidades amostrais submetido ao $m$-ésimo tratamento do primeiro grupo e ao $s$-ésimo tratamento do segundo grupo;

- $\Gamma_{m}$ : o efeito do $m$-ésimo tratamento do primeiro grupo;

- $\Theta_{s}$ : o efeito do s-ésimo tratamento do segundo grupo;

- $\Lambda_{m s}$ : o efeito de interação $m$-ésimo tratamento do primeiro grupo com o $s$-ésimo tratamento do segundo grupo.

Modelo com sinais $\Omega(t), \Gamma_{m}(t), \Theta_{s}(t)$ e $\Lambda_{m s}(t)$

Digamos que o pesquisador está interessado em verificar se existe efeito de interação entre dois grupos de tratamentos previamente fixados em que,

- $\mu_{\ell m s}$ : o nível da $\ell$-ésima unidade amostral dentro do $m$-ésimo tratamento;

- $\Omega(t)$ : o sinal comum entre todas as unidades amostrais; 
- $\Gamma_{m}$ : o sinal (vetor de tamanho $T$ ) em comum entre todas as unidades amostrais do $m$-ésimo tratamento do primeiro grupo, é determinístico com a seguinte restrição para obter a identificabilidade,

$$
\sum_{s=1}^{S} \sum_{m=1}^{M} L_{m s} \Gamma_{m}(t)=0 ;
$$

- $\Theta_{s}$ : o sinal (vetor de tamanho $T$ ) em comum entre todas as unidades amostrais do $s$-ésimo tratamento do segundo grupo, é determinístico com a seguinte restrição para obter a identificabilidade,

$$
\sum_{s=1}^{S} \sum_{m=1}^{M} L_{m s} \Theta_{s}(t)=0 ;
$$

- $\Lambda_{m s}$ : o sinal (vetor de tamanho $T$ ) em comum entre todas as unidades amostrais do $m$-ésimo tratamento do primeiro grupo e $s$-ésimo tratamento do segundo grupo, é determinístico com a seguinte restrição para obter a identificabilidade,

$$
\sum_{s=1}^{S} \sum_{m=1}^{M} L_{m s} \Lambda_{m s}(t)=0
$$

- $\epsilon_{\ell m s}$ são séries estacionárias com média zero.

A transformada de ondaletas do modelo 4.3.3 é dado por

$$
w_{\ell m s}(j, k)=\theta(j, k)+\tau_{m}(j, k)+\eta_{s}(j, k)+\delta_{m s}(j, k)+\varepsilon_{l m s}(j, k),
$$

, com a restrição $\sum_{m=1}^{M} \tau_{m}(j, k)=0, \sum_{s=1}^{S} \eta_{m}(j, k)=0$ e $\sum_{m=1}^{M} \sum_{s=1}^{S} \delta_{m s}(j, k)=0$ para $j$ e $k$ fixos.

Definindo as somas dos quadrados:

$$
\begin{gathered}
S Q_{\Gamma}=\sum_{m=1}^{M} L_{m}\left(\bar{w}_{\bullet m \bullet}(j, k)-\bar{w}_{\bullet \bullet \bullet}(j, k)\right)^{2}, \\
S Q_{\Theta}=\sum_{s=1}^{S} L_{s}\left(\bar{w}_{\bullet \bullet s}(j, k)-\bar{w}_{\bullet \bullet \bullet}(j, k)\right)^{2}, \\
S Q_{\Lambda}=\sum_{s=1}^{S} \sum_{m=1}^{M} L_{m s}\left(\bar{w}_{\bullet m s}(j, k)-\bar{w}_{\bullet m \bullet}(j, k)-\bar{w}_{\bullet \bullet s}(j, k)+\bar{w}_{\bullet \bullet}(j, k)\right)^{2}, \\
S Q_{R}=\sum_{m=1}^{M} \sum_{s=1}^{S} \sum_{l=1}^{L_{m s}}\left(w_{l m s}(j, k)-\bar{w}_{\bullet m s}(j, k)\right)^{2},
\end{gathered}
$$

em que $L_{s}$ é o número de unidades amostrais no $s$-ésimo nível do segundo fator, $L_{m s}$ é o número de unidades amostrais no $m$-ésimo nível do primeiro fator e $s$-ésimo nível do segundo fator, $\bar{w}_{\bullet \bullet \bullet}=\frac{1}{N} \sum_{m=1}^{M} \sum_{s=1}^{S} \sum_{l=1}^{L_{m s}} w_{l m s}$ é média geral, $\bar{w}_{\bullet m \bullet}=\frac{1}{N_{s}} \sum_{s=1}^{S} \sum_{l=1}^{L_{m s}} w_{l m s}$ é a média do $m$-ésimo 
nível do primeiro fator, $\bar{w}_{\bullet \bullet}=\frac{1}{N_{M}} \sum_{m=1}^{M} \sum_{l=1}^{L_{m s}} w_{l m s}$ é a média do $s$-ésimo nível do segundo fator, $\bar{w}_{\bullet m s}(j, k)$ é a média do $m$-ésimo nível do primeiro fator e $s$-ésimo nível do segundo fator.

Para testar a hipótese de interação entre os fatores temos,

$$
H_{0}: \delta_{11}(j, k)=\delta_{12}(j, k)=\ldots=\delta_{M S}(j, k)=0 .
$$

Sob $H_{0}$, as seguintes somas de quadrados convergem para (Montgomery, 2017) e (Pinheiro e Vidakov 1993),

$$
\begin{aligned}
& \frac{S Q_{\Lambda}}{\sigma^{2}} \stackrel{d}{\longrightarrow}, \chi_{(M-1)(S-1)}^{2}, \\
& \frac{S Q_{R}}{\sigma^{2}} \stackrel{d}{\longrightarrow} \chi_{N-M S}^{2} .
\end{aligned}
$$

Para verificar a existência de sinal em comum entre as interações, podemos utilizar a seguinte estatística de teste para cada $j$ e $k$,

$$
F(j, k)=\frac{(N-M S) S Q_{\Lambda}}{(M-1)(S-1) S Q_{R}} \stackrel{d}{\longrightarrow} F_{((M-1)(S-1), N-S M)} .
$$

Um resumo desse procedimento é dado pela Tabela 4.3

Tabela 4.3: ANOVA para testar sinal determinístico de interação entre grupos de tratamentos nos indices $(j, k)$.

\begin{tabular}{lllll}
\hline $\begin{array}{l}\text { Fonte } \\
\text { de va- } \\
\text { riação }\end{array}$ & $\begin{array}{l}\text { Graus de liber- } \\
\text { dade }\end{array}$ & Soma de quadrados $(S Q)$ & Quadrado médio $(Q M)$ & $F$ \\
\hline$\Omega$ & 1 & $N \bar{w}_{\bullet \bullet \bullet}(j, k)^{2}$ & $S Q_{\Omega}$ & $\frac{Q M_{\Omega}}{Q M_{R}}$ \\
$\Gamma$ & $(M-1)$ & $\sum_{s=1}^{M} L_{m}\left(\bar{w}_{\bullet m \bullet} \bullet(j, k)-\bar{w}_{\bullet \bullet \bullet}(j, k)\right)^{2}$ & $S Q_{\Gamma} /(M-1)$ & $\frac{Q M_{\Gamma}}{Q M_{R}}$ \\
$\Theta$ & $(S-1)$ & $\sum_{s=1}^{S} L_{s}\left(\bar{w}_{\bullet \bullet s}(j, k)-\bar{w}_{\bullet \bullet \bullet}(j, k)\right)^{2}$ & $S Q_{\Theta} /(S-1)$ & $\frac{Q M_{\Theta}}{Q M_{R}}$ \\
$\Lambda$ & $(M-1)(S-1)$ & $\sum_{s=1}^{S} \sum_{m=1}^{M} L_{m s}\left(\bar{w}_{\bullet m s}(j, k)-\bar{w}_{\bullet} \bullet(j, k)-\bar{w}_{\bullet \bullet}(j, k)+\bar{w}_{\bullet \bullet \bullet}(j, k)\right)^{2}$ & $S Q_{\Lambda} /(M-1)(S-1)$ & $\frac{Q M_{\Lambda}}{Q M_{R}}$ \\
Resíduo & $(N-M S)$ & $\sum_{m=1}^{M} \sum_{s=1}^{S} \sum_{\ell=1}^{L_{m s}}\left(w_{\ell m s}(j, k)-\bar{w}_{\bullet m s}(j, k)\right)^{2}$ & $S Q_{R} /(N-M S)$ & \\
\hline Total & $N$ & $\sum_{m=1}^{M} \sum_{s=1}^{S} \sum_{\ell=1}^{L_{m s}} w_{\ell m s}(j, k)^{2}$ & \\
\hline
\end{tabular}




\section{Capítulo 5}

\section{Simulação}

Nesse capítulo, serão apresentadas simulações da análise de variância no contexto da TDO, TDOND e TDF com o objetivo de ilustrar alguns resultados teóricos de sessões anteriores.

\subsection{Gerando os dados com sinais diferentes}

Para a simulação, vamos considerar a situação onde há apenas um fator. Foram utilizados três sinais diferentes (níveis do fator), as funções doppler, blocks, heavisine, todas do pacote waveslim (Whitcher, 2020) no software $R$ (R Core Team, 2020). O sinal para cada nível simulado é dado pelas seguintes funções.

$$
\begin{aligned}
& f_{1}(t)=\operatorname{blocks}(t) \\
& f_{2}(t)=\text { blocks }(t)+\text { doppler }(t) \\
& f_{3}(t)=\operatorname{blocks}(t)+\text { heavisine }(t) .
\end{aligned}
$$

Ou, de maneira mais resumida,

$$
\mu_{m}(t)=\Omega(t)+\Gamma_{m}(t), m=1,2,3, t=0,1, \ldots, 511,
$$

em que, $\Omega(t)$ é o sinal em comum dos três níveis, ou seja, a função blocks $(t), \Gamma_{1}(t)=0$, $\Gamma_{2}(t)=$ doppler $(t), \Gamma_{3}(t)=$ heavisine $(t)$. A ilustração dos sinais é apresentada a seguir.
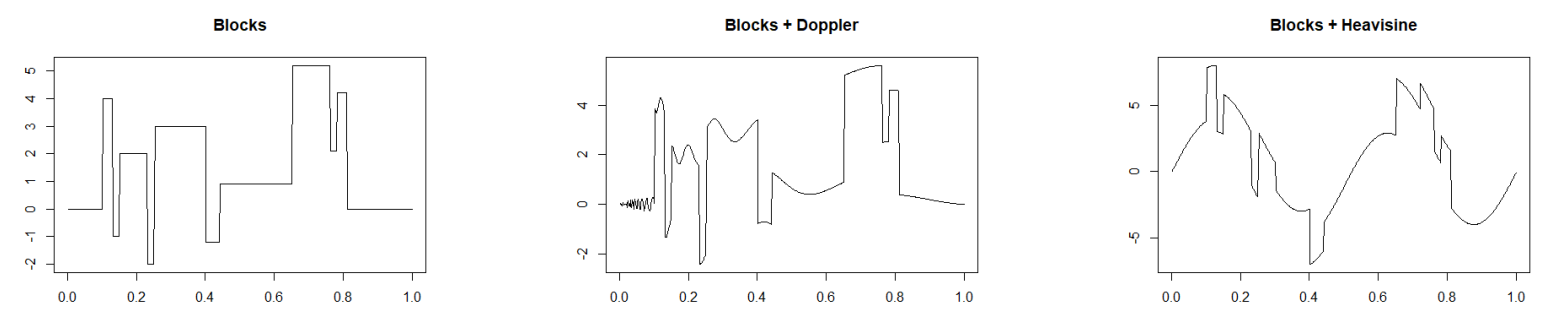

Figura 5.1: Sinais das séries simuladas.

Foram simuladas 1000 séries temporais de tamanho $T=512$ para cada nível do fator, 
totalizando 3000 séries temporais. Os dados simulados possuem a seguinte forma:

$$
y_{\ell m}(t)=\Omega(t)+\Gamma_{m}(t)+\epsilon_{\ell m}(t),
$$

em que, $m=1,2,3, l=1,2, \ldots, 1000, t=0,2, \ldots, 511, \epsilon_{\ell m}(t)$ é um termo de erro com distribuição normal, média zero e desvio padrão 0,25 .

\section{- Transformada discreta de ondaletas}

$\mathrm{Na}$ aplicação da análise de variância utilizando da transformada discreta de ondaletas, foram incluídos todos os níveis de resolução $\left(J_{0}=0\right)$. A base de ondaletas utilizada foi a Least Asymmetic LA8, também chamada de symmlets com filtro de tamanho 8.

Após a transformação, o modelo pode ser representado como,

$$
w_{\ell m}(j, k)=\theta(j, k)+\tau_{m}(j, k)+\varepsilon_{\ell m}(j, k),
$$

em que, $m=1,2,3, \ell=1,2, \ldots, 1000, j=0,1, \ldots, 8, k=0,2, \ldots, 2^{j}-1$. Para verificar a hipótese de existência de sinal em comum entre os níveis vamos testar para cada par $(j, k)$

$$
\begin{aligned}
& H_{0}: \quad \tau_{1}(j, k)=\tau_{2}(j, k)=\tau_{3}(j, k)=0, \\
& H_{1}: \quad \text { Pelo menos um } \tau_{m}(j, k) \neq 0, m=1,2,3 .
\end{aligned}
$$

Os $p$-valores da ANOVA para cada par $(j, k)$ são exibidos abaixo, os pontos marcados em vermelho são significativos ao nível de significância de $5 \%$.
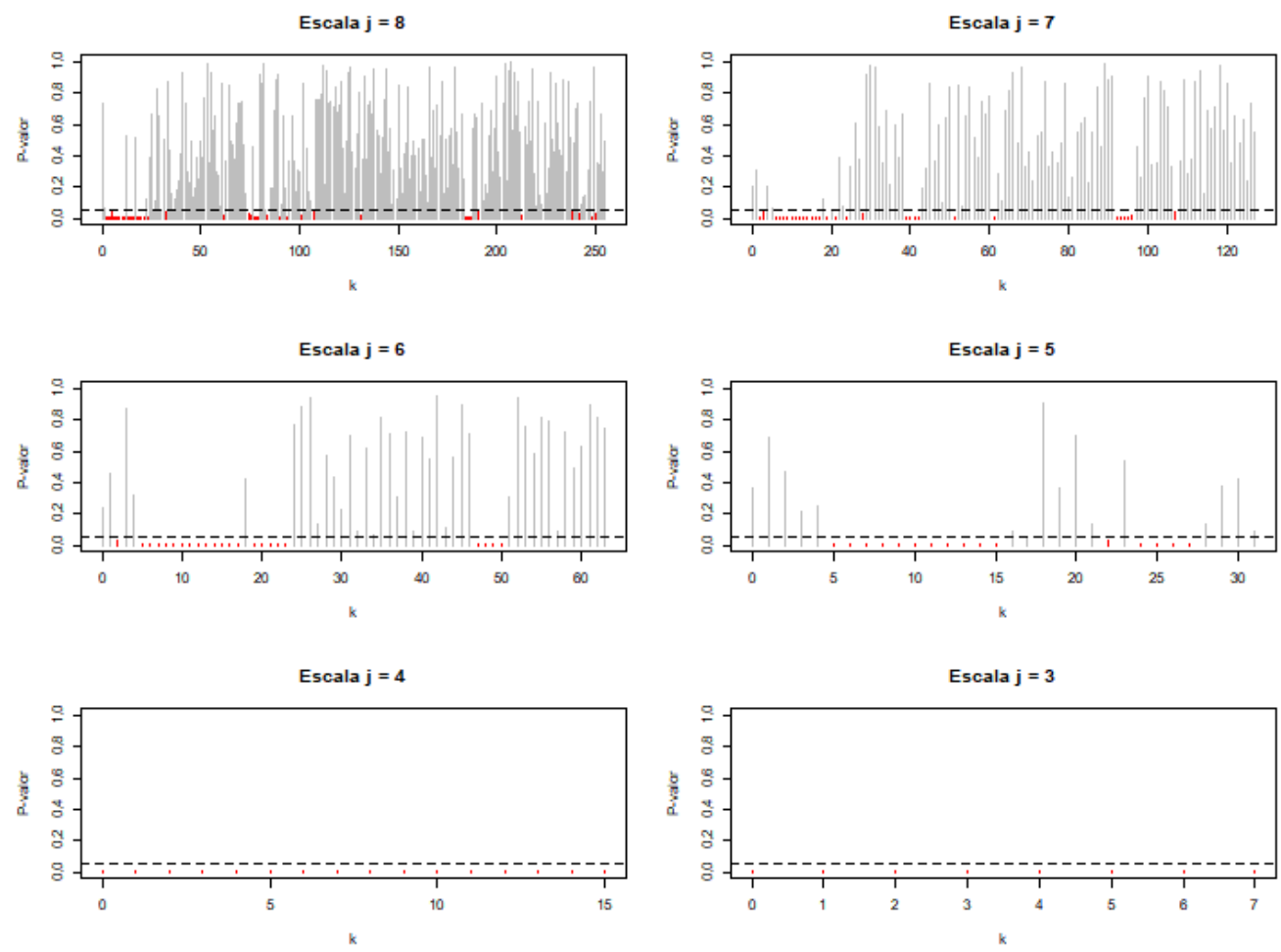

Figura 5.2: Simulação TDO: p-valores exibidos por nível de resolução $J=8,7,6,5,4,3$. 

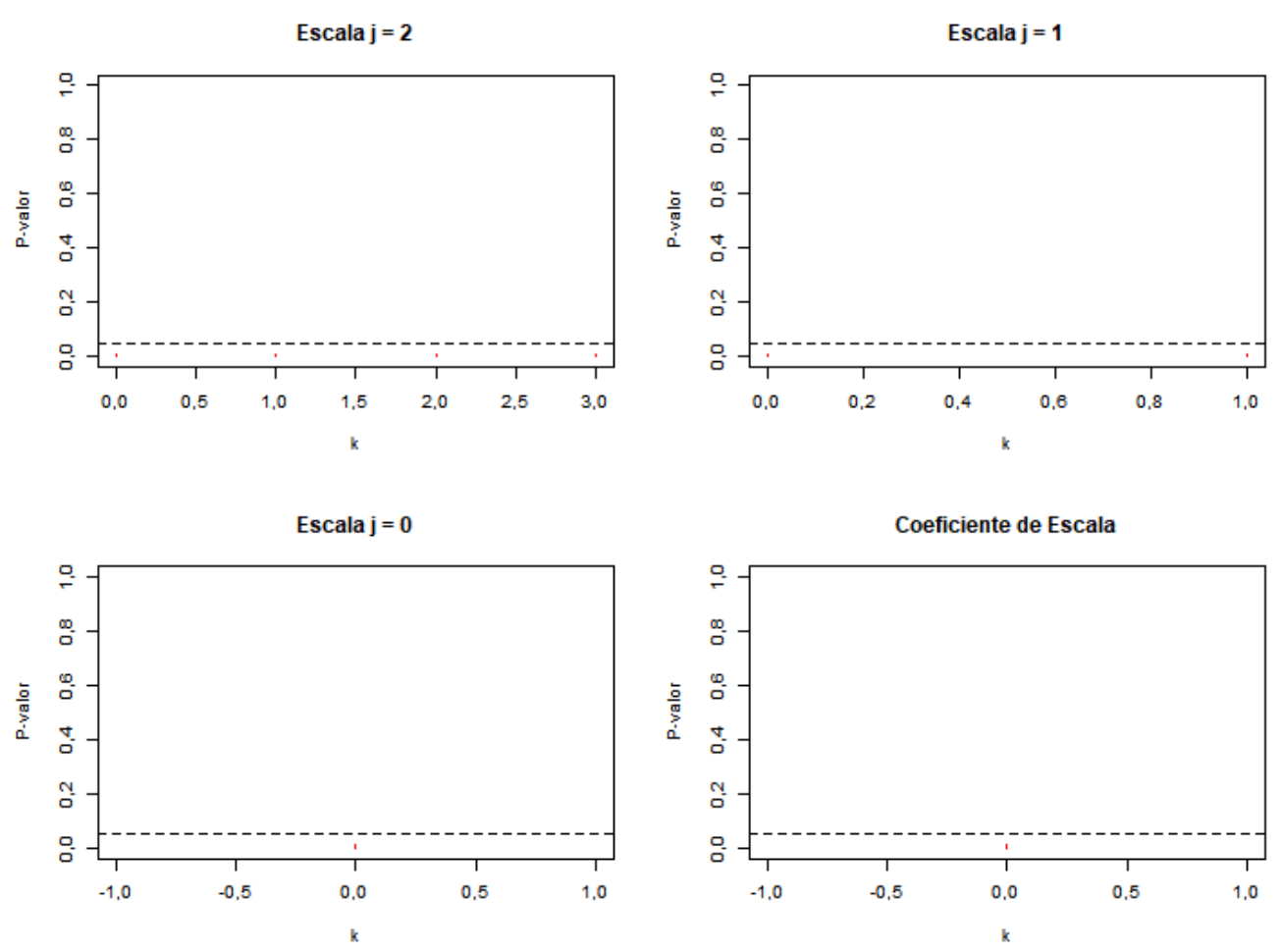

Figura 5.3: Simulação TDO: p-valores exibidos por nível de resolução $J=2,1,0$.

\section{- Transformada discreta de ondaletas não decimada}

Na aplicação da análise de variância utilizando da transformada discreta de ondaleta não decimada foi aplicada a transformação (4.38) para atenuar a autocorrelação dos coeficientes da transformada de ondaletas não decimada. Devido à autocorrelação presente nos coeficientes, o menor nível de resolução usado foi $J_{0}=6$ e foram considerados apenas os coeficientes dos detalhes. Foi utilizada a ondaleta Least Asymmetic LA8. O modelo pode ser representado como

$$
w_{\ell m}\left(j, k^{\prime}\right)=\theta\left(j, k^{\prime}\right)+\tau_{m}\left(j, k^{\prime}\right)+\varepsilon_{\ell m}\left(j, k^{\prime}\right),
$$

em que, $m=1,2,3, l=1,2, \ldots, 1000, j=1,2,3, k^{\prime}=0,1, \ldots, 255$. Para verificar a hipótese de existência de sinal em comum entre os níveis vamos testar para cada par $\left(j, k^{\prime}\right)$

$$
\begin{aligned}
& H_{0}: \quad \tau_{1}\left(j, k^{\prime}\right)=\tau_{2}\left(j, k^{\prime}\right)=\tau_{3}\left(j, k^{\prime}\right)=0, \\
& H_{1}: \quad \text { Pelo menos um } \tau_{m}\left(j, k^{\prime}\right) \neq 0, m=1,2,3 .
\end{aligned}
$$

Os $p$-valores da ANOVA para cada par $\left(j, k^{\prime}\right)$ são exibidos abaixo, os pontos marcados em vermelho são significativos ao nível de significância de $5 \%$. 


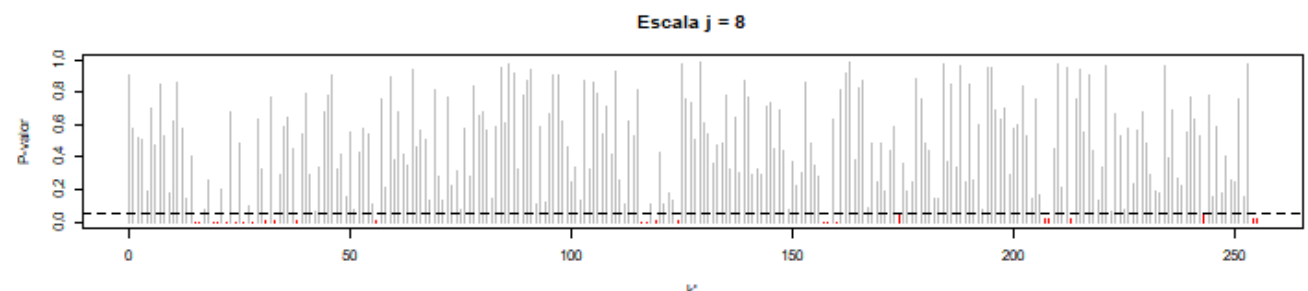

Escala $\mathbf{j}=7$

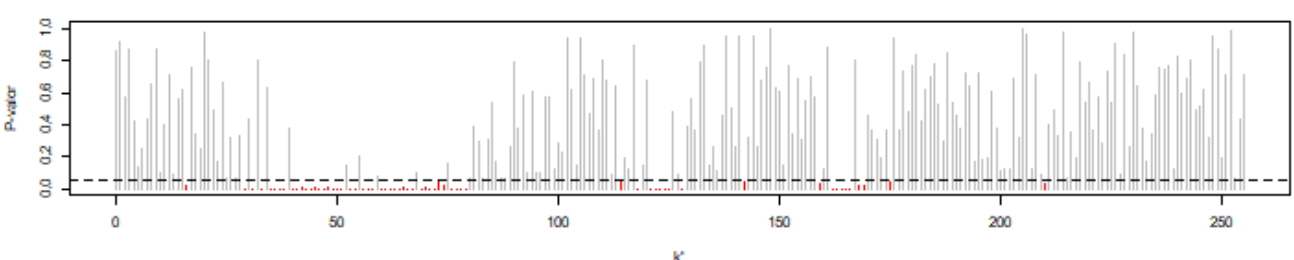

Escala $\mathbf{j}=6$

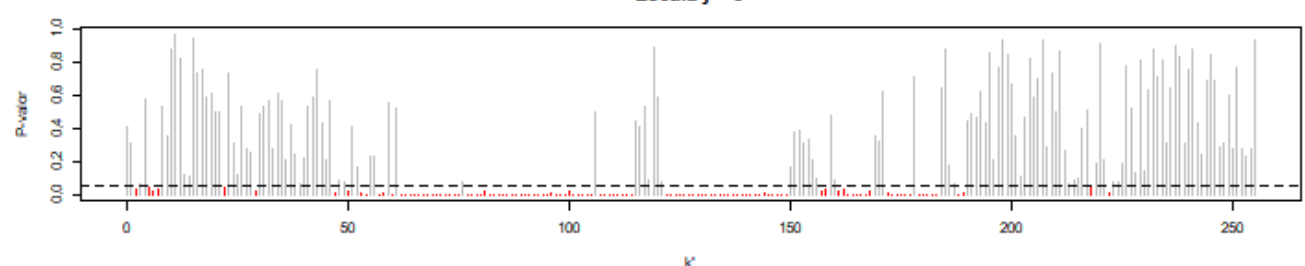

Figura 5.4: Simulação TDOND: p-valores exibidos por nivel de resolução.

\section{- Transformada discreta de Fourier}

No caso da transformada discreta de Fourier, o modelo pode ser representado como,

$$
d_{\ell m}(\lambda)=\theta(\lambda)+\tau_{m}(\lambda)+\varepsilon_{\ell m}(\lambda),
$$

em que, $m=1,2,3, l=1,2, \ldots, 1000, \lambda=\lambda(k)=2 \pi k / T, k=0,1, \ldots, T / 2$. Para verificar a hipótese de existência de sinal em comum entre os níveis vamos testar para cada par frequência $\lambda$

$$
\begin{array}{cc}
H_{0}: & \tau_{1}(\lambda)=\tau_{2}(\lambda)=\tau_{3}(\lambda)=0, \\
H_{1}: & \text { Pelo menos um } \tau_{m}(\lambda) \neq 0, m=1,2,3 .
\end{array}
$$

Abaixo são ilustrados os $p$-valores para ANOVA no caso da transformada de Fourier. As frequências significativas ao nível de $5 \%$ são exibidas bem vermelho. 


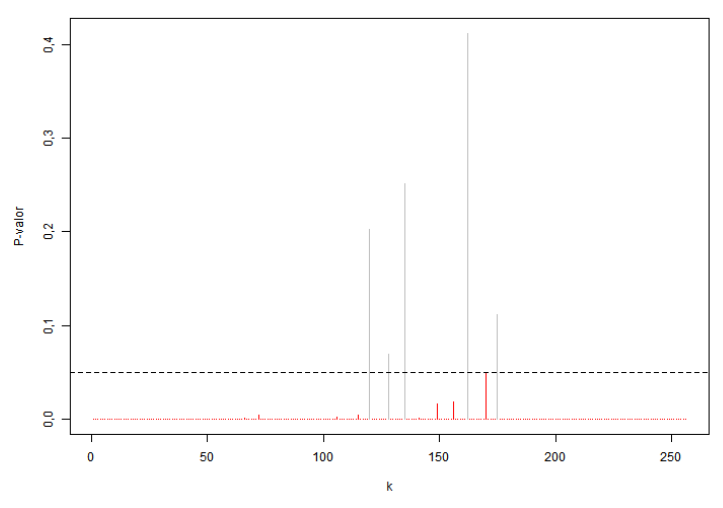

Figura 5.5: Sinais diferentes: p-valores da transformada de Fourier.

\subsubsection{Poder do teste}

A função poder de um teste de hipótese com respeito a uma região de rejeição $\boldsymbol{R}$ é definida por $\beta(\boldsymbol{\theta})=P(\mathbf{X} \in \mathbf{R} \mid \boldsymbol{\theta})$ (Casella e Berger, 2002), em outras palavras, é a probabilidade de rejeitar $H_{0}$ dados valores do vetor de parâmetros $\boldsymbol{\theta}$. Nessa simulação, $\boldsymbol{\theta}=\boldsymbol{\theta}(j, k)=$ $\left(\Gamma_{1}(j, k), \Gamma_{2}(j, k), \Gamma_{3}(j, k)\right)$. No caso da TDO, $\boldsymbol{\theta}=\boldsymbol{\theta}\left(j, k^{\prime}\right)=\left(\Gamma_{1}\left(j, k^{\prime}\right), \Gamma_{2}\left(j, k^{\prime}\right), \Gamma_{3}\left(j, k^{\prime}\right)\right)$. No caso da TDOND e $\boldsymbol{\theta}=\boldsymbol{\theta}(\lambda)=\left(\Gamma_{1}(\lambda), \Gamma_{2}(\lambda), \Gamma_{3}(\lambda)\right)$ no caso da TDF. Após realizadas 1000 simulações de cada teste, podemos obter o poder do teste de maneira empírica por meio das frequências em que as estatísticas de teste pertencem a região de rejeição. Os resultados são ilustrados nos gráficos a seguir.

\section{- Transformada discreta de ondaletas}
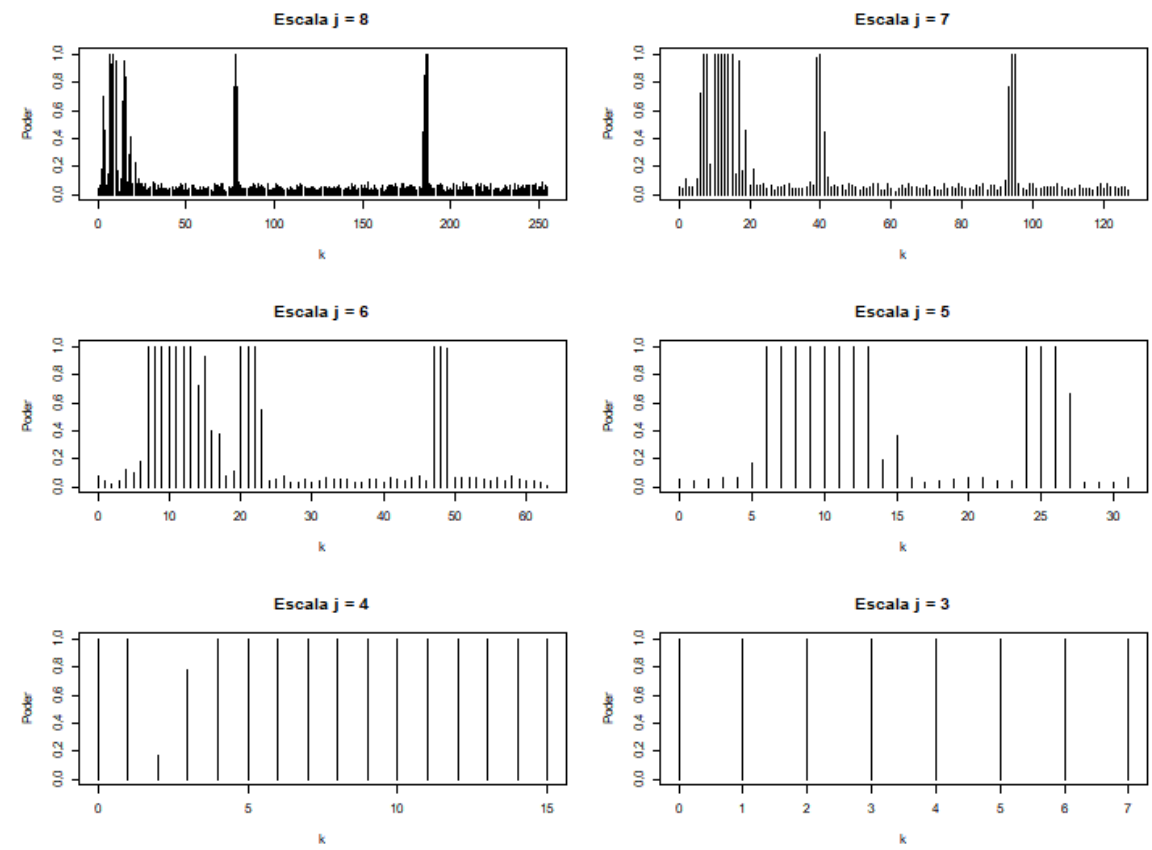

Figura 5.6: Poder do teste empírico para transformada discreta de ondaletas nas resoluçôes $J=$ $8,7,6,5,4,3$. 

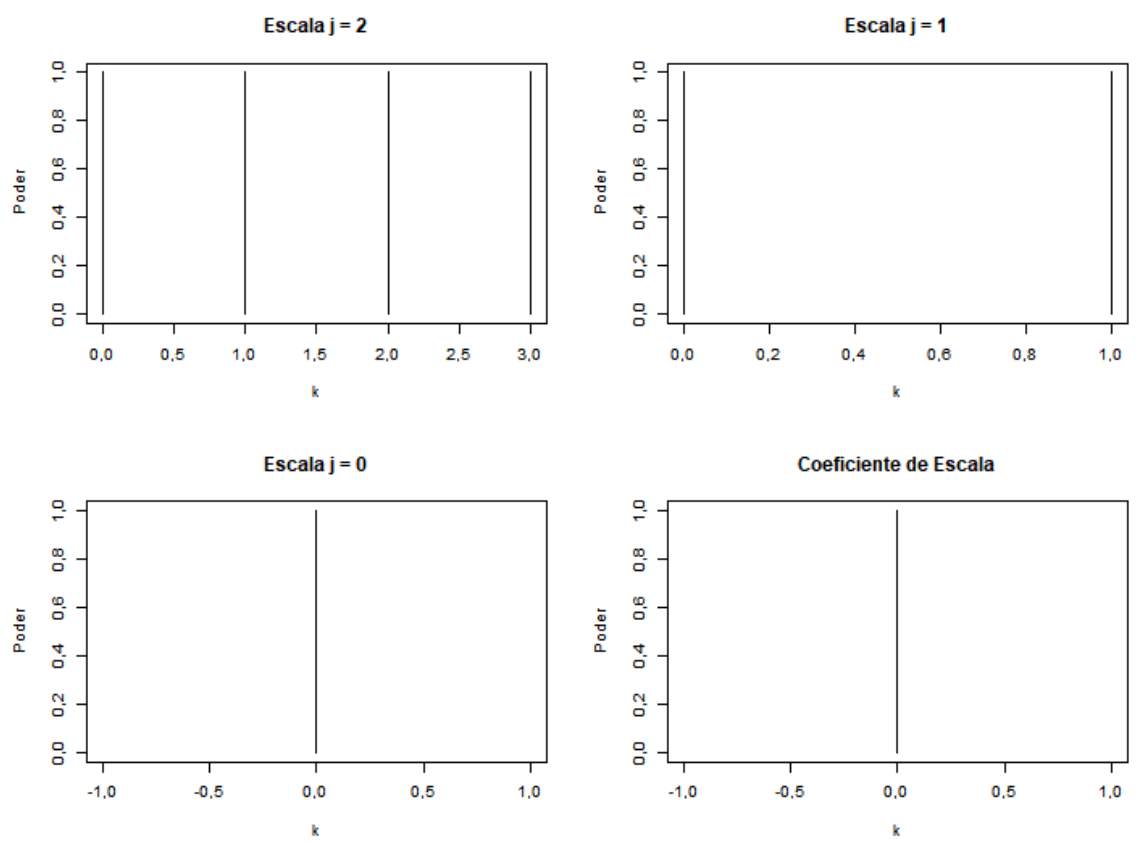

Figura 5.7: Simulação TDO: Poder do teste empírico para transformada discreta de ondaletas nas resoluções $J=2,1,0$.

Nas escalas mais finas, poucos coeficientes possuem alto poder. O alto poder se concentra mais nos coeficientes relacionados ao inicio das séries em que possui sinal com alta frequência. A medida que se avança para as escalas mais grossas, o poder do teste fica bastante alto para quase todos os coeficientes,

- Transformada discreta de ondaletas não decimada
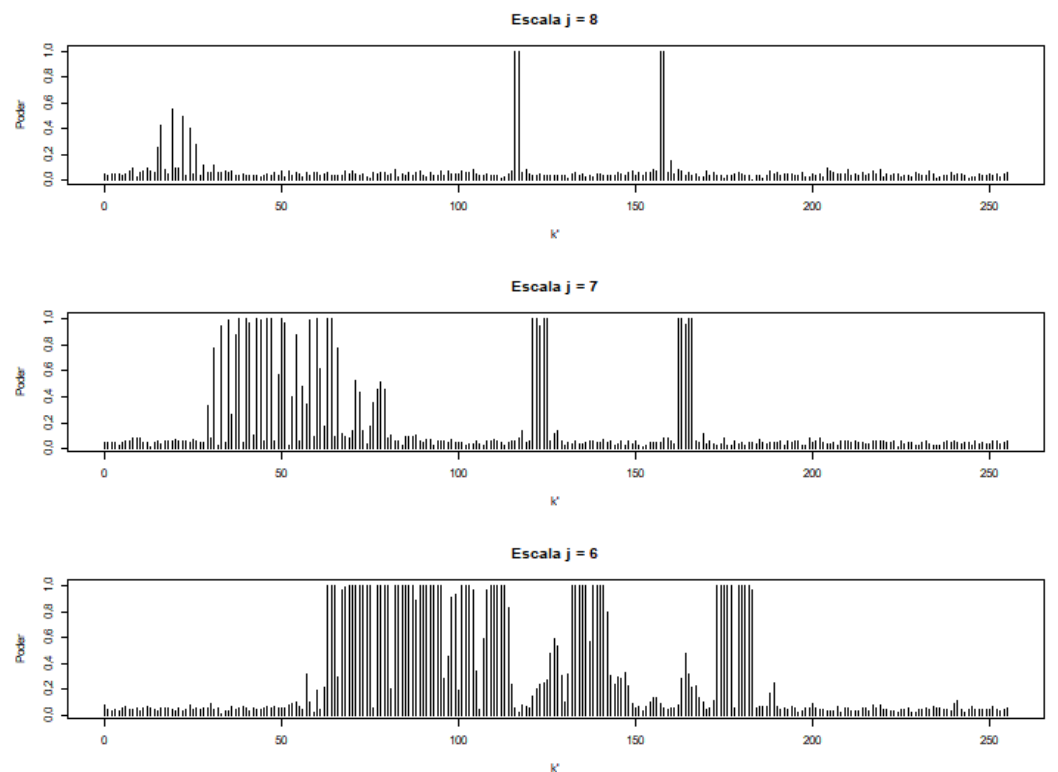

Figura 5.8: Simulação TDOND: Poder do teste empírico para transformada discreta de ondaletas não decimada. 
Percebe-se que na escala mais fina $(j=8)$, assim como ocorre na TDO, poucos coeficientes possuem poder de teste alto, e a medida em que a escala fica mais "grossa", a quantidade de testes com alto poder aumenta. Esse fato ocorre pois a escala $j=6$ está mais relacionado com a tendência da série, alterações a médio prazo, do que a escala $j=8$, que está mais relacionada com alterações de curto prazo.

\section{- Transformada discreta de Fourier}

Para efeito de comparação, abaixo é exibido o gráfico da função do poder empírico para a aplicação da transformada discreta de Fourier.

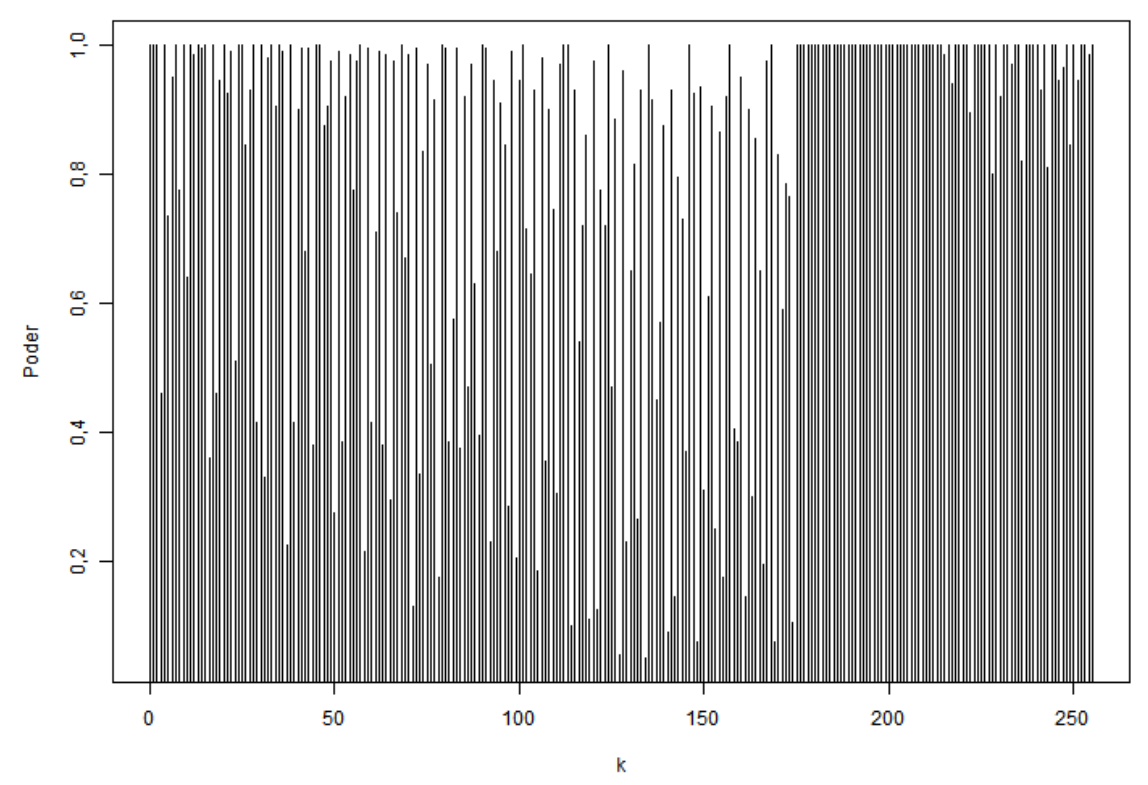

Figura 5.9: simulação TDF: Poder do teste empírico para transformada discreta de Fourier.

Note que para frequências mais altas a função poder possui mais pontos com poder alto, mas para pontos com frequências mais baixas, a quantidade de pontos com poder próximo ao valor 1 diminui. Os resultados obtidos, tanto para ondaletas quanto para Fourier parecem divergir nesse pontos, pois nos casos das transformadas discretas de ondaletas os testes tendem a ser mais poderosos em escalas com menores frequências (variações de médio e longo prazo), já no caso da transformada discreta de Fourier, a análise de variância detecta melhor as diferenças em frequências maiores.

\subsection{Gerando os dados com sinais iguais}

Agora será simulado o cenário em que todos os níveis possuem o mesmo sinal em comum, ou seja, a função blocks $(t)$. Novamente, foram simuladas 1000 séries para cada nível do fator, e em seguida foram feitas análises de variância para detectar os efeitos dos grupos, os resultados para as três técnicas são exibidos a seguir.

\section{- Transformada discreta de ondaletas}

Abaixo são ilustrados os $p$-valores para ANOVA no caso da transformada discreta de ondaletas 

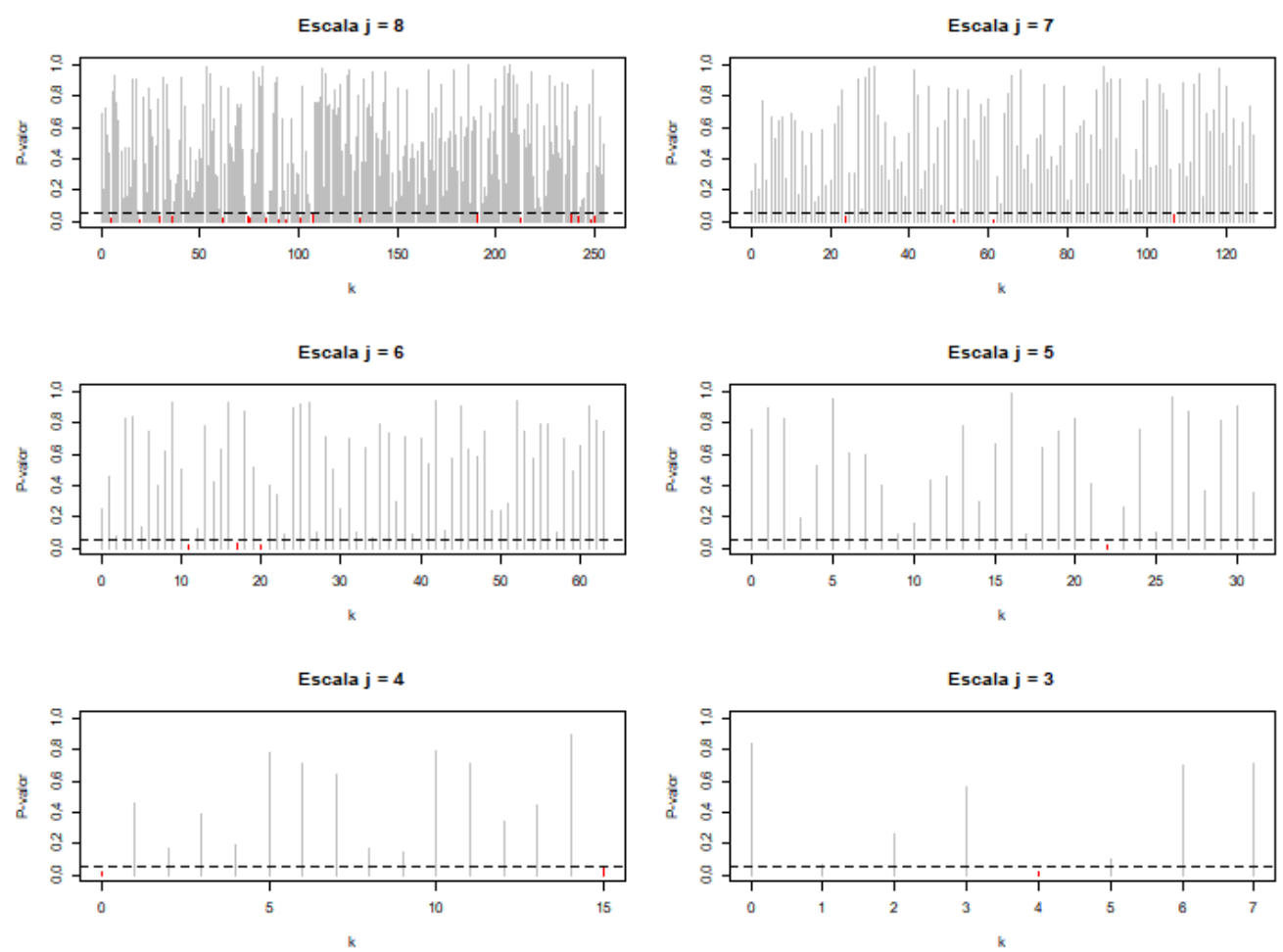

Figura 5.10: Simulaçẫo TDO: p-valores exibidos por nivel de resoluçâo $J=8,7,6,5,4,3$.
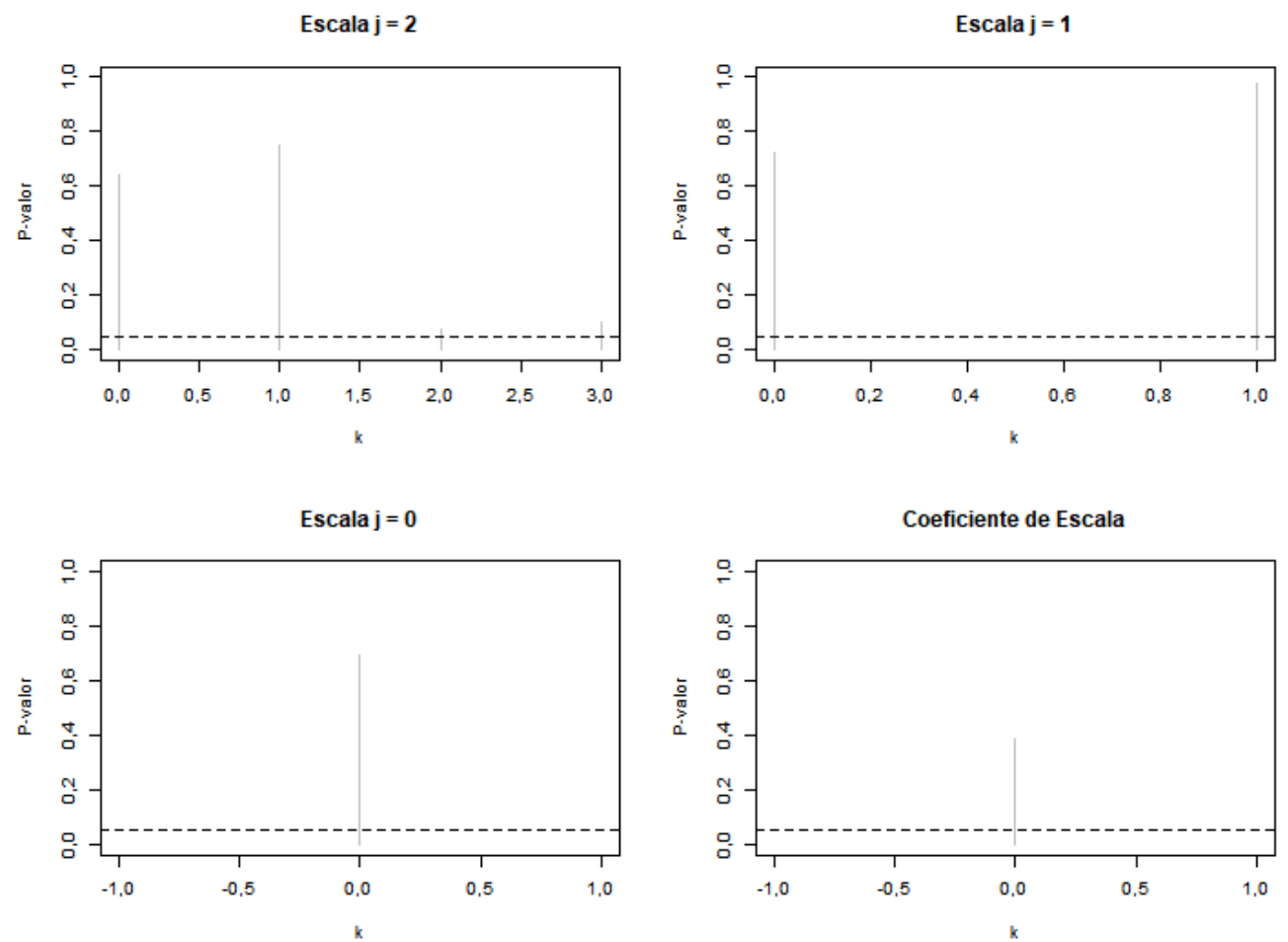

Figura 5.11: Simulação TDO: p-valores exibidos por nível de resolução $J=2,1,0$.

\section{- Transformada discreta de ondaletas não decimada}

Abaixo são ilustrados os $p$-valores para ANOVA no caso da transformada discreta de 
ondaletas não decimada.
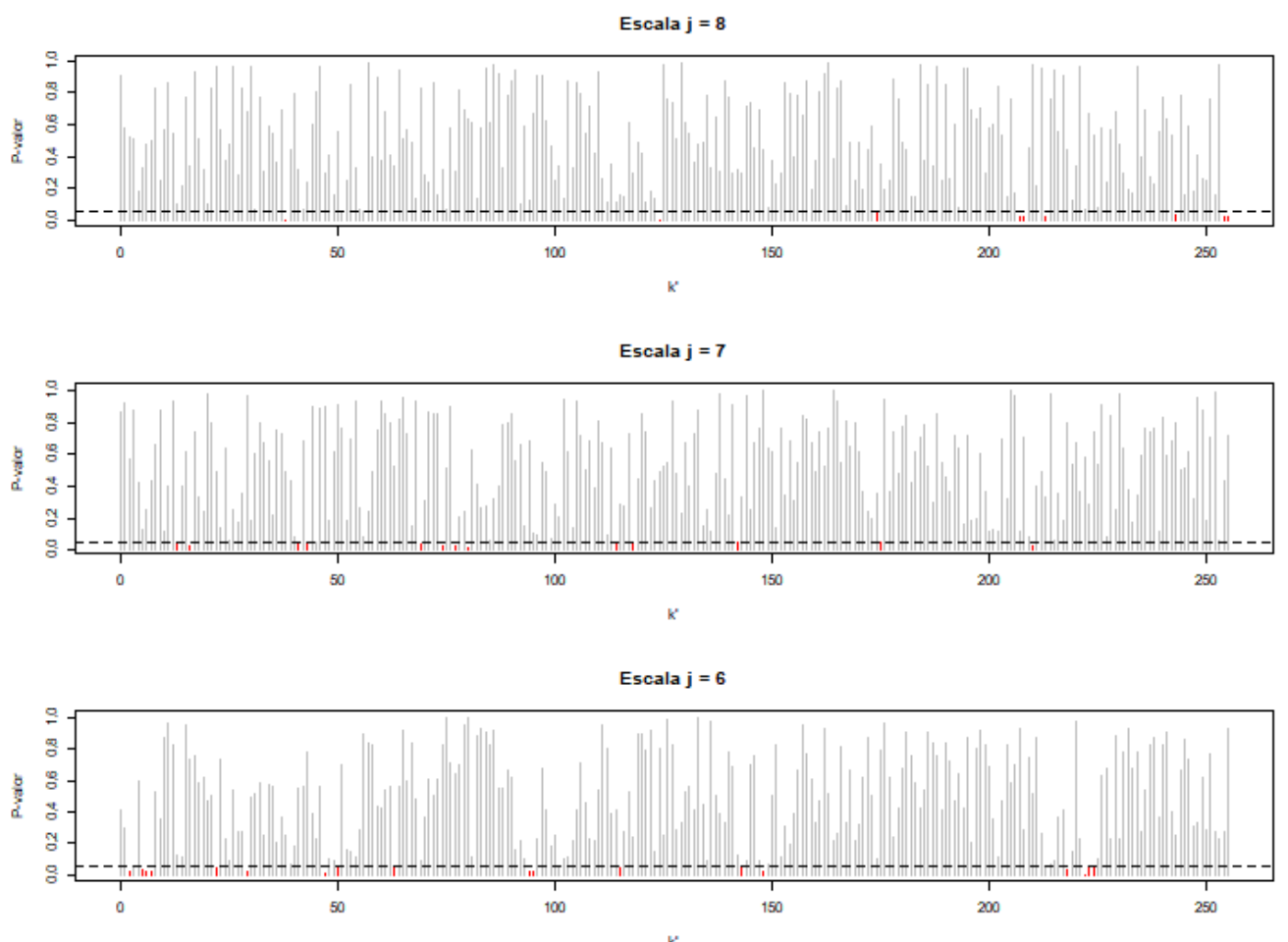

Figura 5.12: Simulação TDOND: p-valores exibidos por nível de resolução.

\section{- Transformada discreta de Fourier}

Abaixo são ilustrados os $p$-valores para ANOVA no caso da transformada de Fourier.

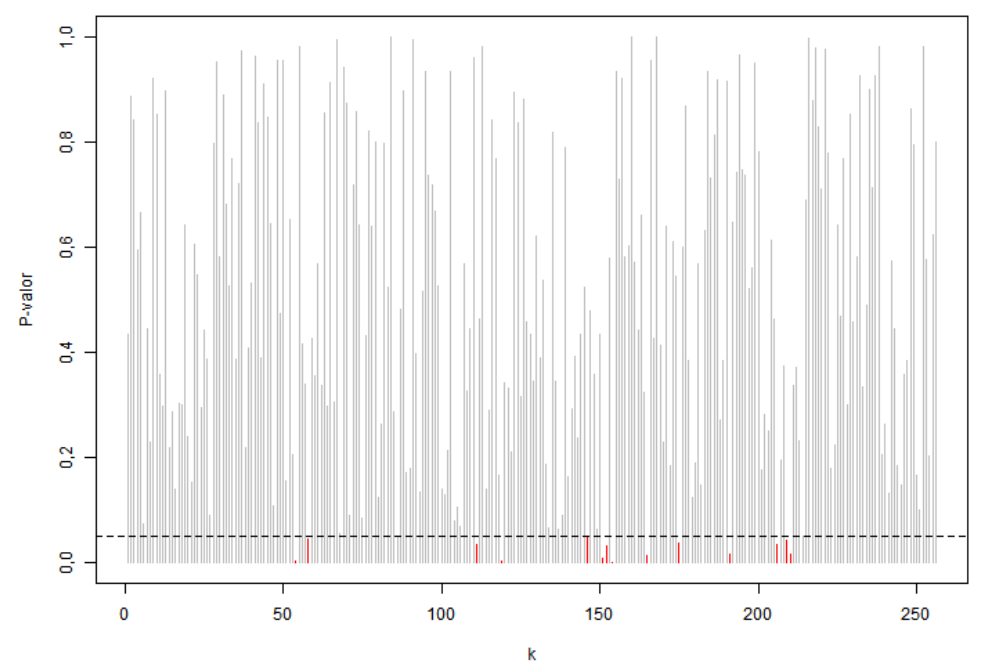

Figura 5.13: Simulação TDF: p-valores da transformada de Fourier.

Como era esperado, cerca de $5 \%$ dos pontos ficaram com seus $p$-valores abaixo do nível de significância. Supondo $H_{0}$ verdadeiro (hipótese de sinais iguais) as estatísticas de testes 
seguem distribuição F com parâmetros 2 e 2997 nos casos da TDO e TDOND e distribuição F com parâmetros 4 e 5994 no caso da TDF. A seguir são exibidos os histogramas contendo as estatísticas de testes observadas e junto foram exibidas as distribuições teóricas.
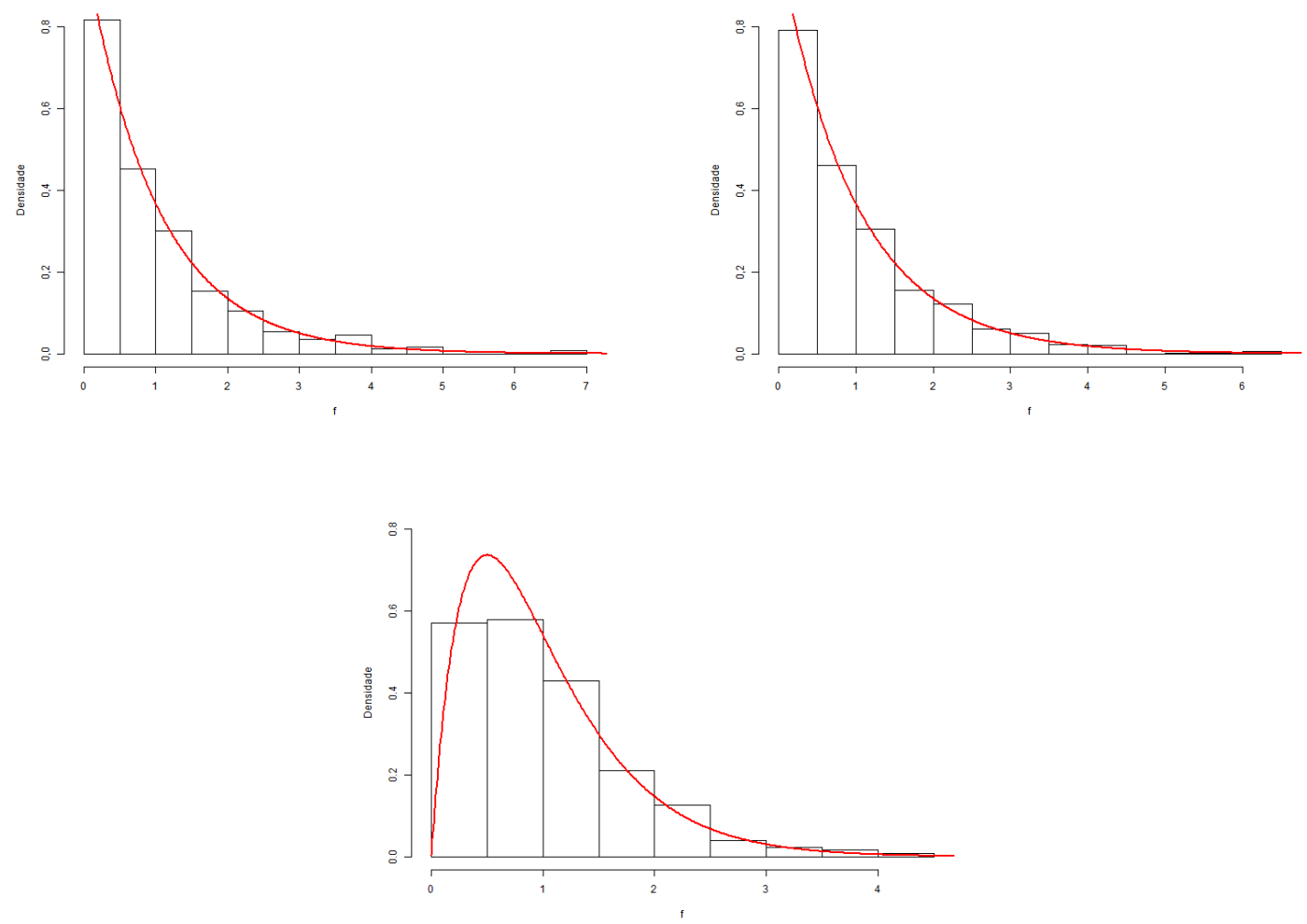

Figura 5.14: Histograma das estatísticas de teste observadas junto com a distribuição teórica (TDO na parte superior esquerda, TDOND na parte superior direita e Fourier na parte inferior).

Com isso, ilustramos resultados teóricos mostrados em capítulos anteriores. Com o intuito de se aproximar mais a um cenário realista, foram testados também cenários com um número menor de séries em cada grupo: 50 e 100 séries por grupo. Os resultados obtidos foram similares às simulações apresentadas anteriormente. 


\section{Capítulo 6}

\section{Aplicação}

Nesse capítulo serão abordadas as aplicações das técnicas de análise de variância para séries temporais descritas nos capítulos anteriores.

\subsection{Banco de dados}

A base de dados utilizada possui informações sobre pico de fluxo expiratório (PFE), característica relacionada ao sistema respiratório, de crianças e adolescentes entre 8 e 15 anos e mais dois fatores: primeiro, se o indivíduo foi diagnosticado com asma e segundo se é exposto ao fumo de terceiros no seu domicílio. A amostra é composta por 253 indivíduos acompanhados por cerca de 65 dias.

Nas Figuras 6.1, 6.2, 6.3 e 6.4 são apresentados alguns exemplos das séries estratificadas por condição de asma e exposição ao fumo domiciliar. As séries não apresentam aspecto estacionário. 

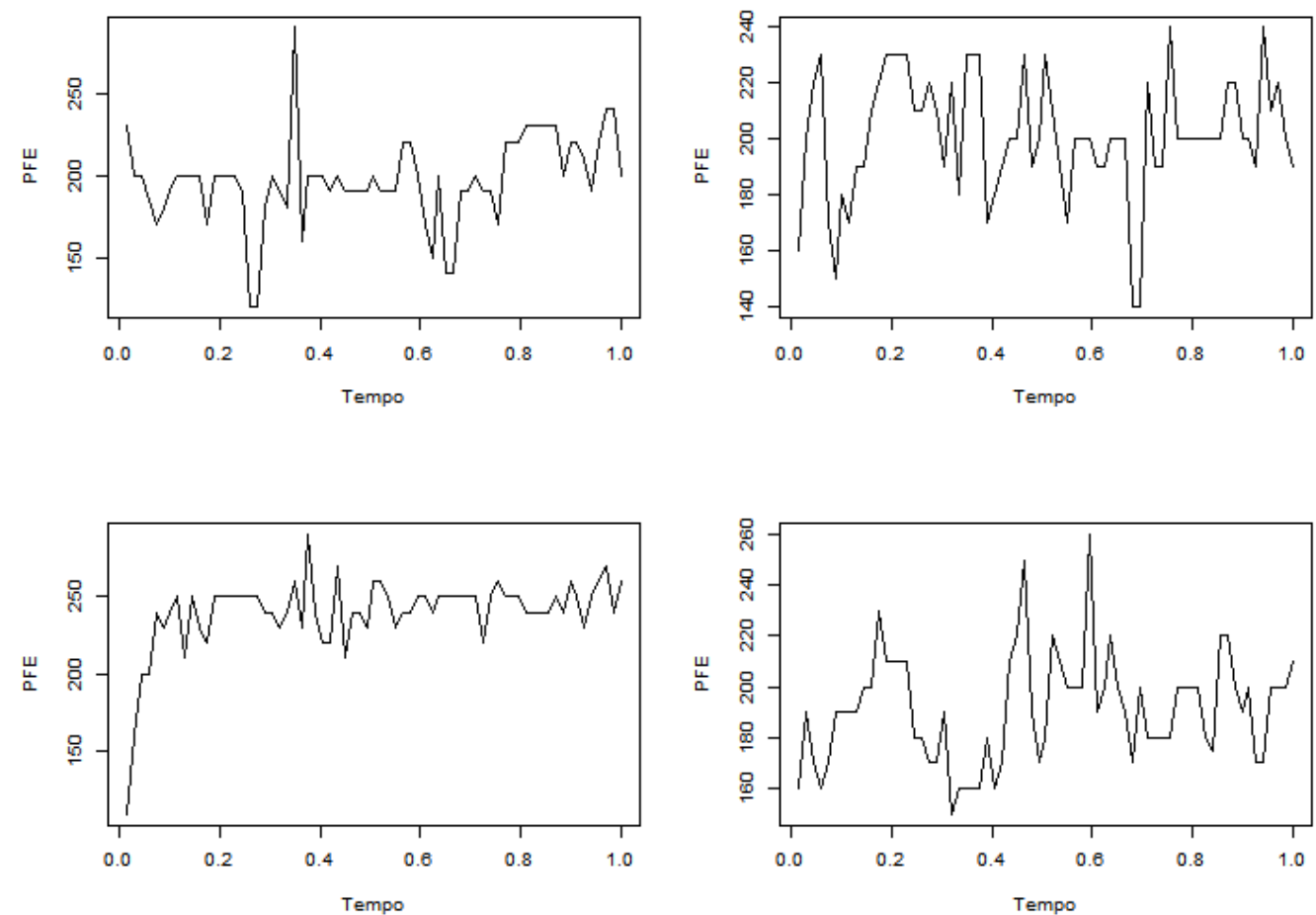

Figura 6.1: Amostra das séries dos indivíduos sem a condição de asma e não expostos ao fumo domiciliar.

\subsection{Análise de variância utilizando Fourier}

Considerando os asma, fumo domiciliar e a interação entre eles, temos o seguinte modelo de análise de variância.

$Y_{\ell m s}(t)=\mu_{\ell m s}+\Omega(t)+\Gamma(t) \times I\left(A s m a_{\ell m s}\right)+\Theta(t) \times I\left(F u m o_{\ell m s}\right)+\Lambda(t) \times I\left(A s m a_{\ell m s}\right) I\left(F u m o_{\ell m s}\right)+\epsilon_{\ell m s}(t)$,

em que $\ell=1, \ldots, L_{m s}, m=1,2, s=1,2, t=0, \ldots, T-1$, de tal forma que $Y_{\ell m s}(t)$ é o Pico de fluxo expiratório da $\ell$-ésima unidade amostral dentro dos níveis $m$ e $s$ no instante $t$, $\mu_{\ell m s}$ é o nível da série, $\Omega(t)$ é o sinal em comum entre as séries, $\Gamma(t)$ é o efeito da asma no instante $t, \Theta(t)$ é o efeito do fumo domiciliar no instante $t, \Lambda(t)$ é o efeito da interação entre asma e fumo domiciliar no instante $t, \epsilon_{\ell m s}(t)$ é um termo de erro aleatório.

No caso da transformada discreta de Fourier, o modelo pode ser reescrito da seguinte maneira:

$d_{\ell m s}(\lambda)=\theta(\lambda)+\tau(\lambda) \times I\left(\right.$ Asma $\left._{\ell m s}\right)+\eta(\lambda) \times I\left(F u m o_{\ell m s}\right)+\delta(\lambda) \times I\left(A s m a_{\ell m s}\right) I\left(F u m o_{\ell m s}\right)+\varepsilon_{\ell m s}(\lambda)$

em que $d_{\ell m s}(\lambda)$ é a transformada do pico de fluxo expiratório da $\ell$-ésima unidade amostral dentro dos níveis $m$ e $s$ na frequência $\lambda, \theta(\lambda)$ é o sinal em comum na frequência $\lambda, \tau$ é o efeito da asma na frequência $\lambda, \eta(\lambda)$ é o efeito do fumo domiciliar na frequência $\lambda, \delta(\lambda)$ é o efeito da interação entre asma e fumo domiciliar na frequência $\lambda, \varepsilon_{\ell m s}(\lambda)$ é um termo de erro aleatório.

Primeiramente, iremos testar se o efeito de interação entre o fumo e a asma são significativos ou não, 

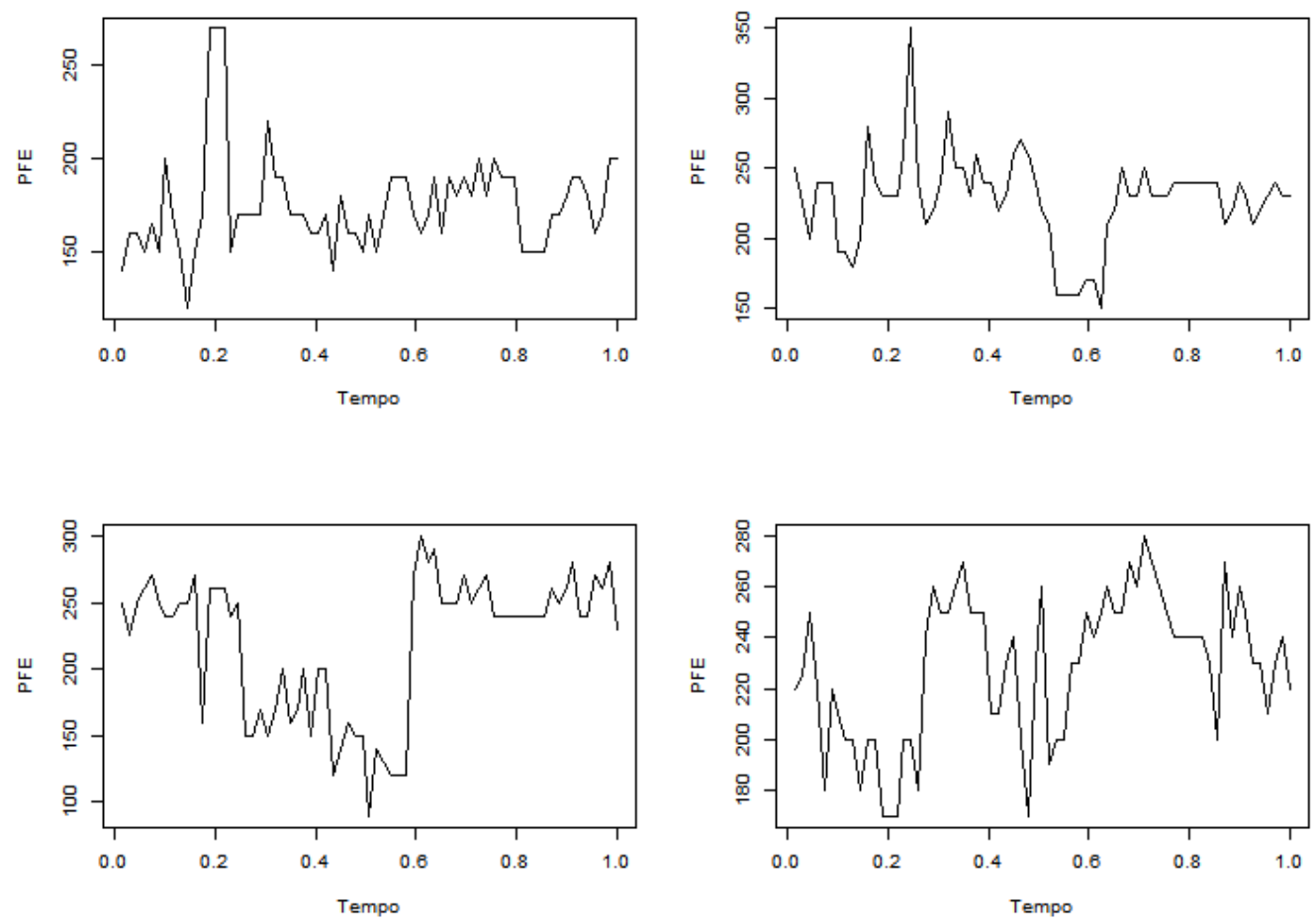

Figura 6.2: Amostra das séries dos indivíduos com a condição de asma e não expostos ao fumo domiciliar.

$$
\left\{\begin{array}{l}
H_{0}: \delta(\lambda)=0, \\
H_{1}: \delta(\lambda) \neq 0 .
\end{array}\right.
$$

Os resultados para as frequências $\lambda(k)=2 \pi k / T$ são apresentados na Figura 6.5 e na Tabela 6.1

Tabela 6.1: TDF: p-valores dos testes de interação para cada frequência $\lambda(k)$, frequências significativas estão em destaque.

\begin{tabular}{c|rrrrrrrr}
\hline$\lambda$ & $0,00 \pi$ & $0,03 \pi$ & $0,06 \pi$ & $0,09 \pi$ & $0,12 \pi$ & $0,16 \pi$ & $0,19 \pi$ & $0,22 \pi$ \\
p-valor & 0,98 & 0,75 & 0,75 & 0,43 & 0,52 & 0,86 & 0,88 & 0,45 \\
\hline$\lambda$ & $0,25 \pi$ & $0,28 \pi$ & $0,31 \pi$ & $0,34 \pi$ & $0,38 \pi$ & $0,41 \pi$ & $0,44 \pi$ & $0,47 \pi$ \\
p-valor & 0,48 & 0,72 & 0,57 & 0,31 & 0,86 & 0,06 & 0,07 & 0,81 \\
\hline$\lambda$ & $0,50 \pi$ & $0,53 \pi$ & $0,56 \pi$ & $0,59 \pi$ & $0,62 \pi$ & $0,66 \pi$ & $0,69 \pi$ & $0,72 \pi$ \\
p-valor & 0,83 & 0,12 & 0,08 & 0,43 & 0,75 & 0,47 & $\mathbf{0 , 0 3}$ & 0,83 \\
\hline$\lambda$ & $0,75 \pi$ & $0,78 \pi$ & $0,81 \pi$ & $0,84 \pi$ & $0,88 \pi$ & $0,91 \pi$ & $0,94 \pi$ & $0,97 \pi$ \\
p-valor & 0,14 & $\mathbf{0 , 0 4}$ & 0,32 & 0,93 & 0,25 & $\mathbf{0 , 0 4}$ & 0,47 & 0,55 \\
\hline
\end{tabular}

Pela Figura 6.5 e pela Tabela 6.1, nos testes para verificar o efeito de interação do fumo com a condição de asma no PFE, as frequências $0,69 \pi, 0,78 \pi$ e $0,91 \pi$ foram consideradas significativas ao nível de $5 \%$. Sugerindo que a interação entre exposição à fumaça no ambiente e condição de asma causa diferenças no PFE a curto prazo dos indivíduos expostos. Na literatura médica, há consenso que indivíduos asmáticos possuem mais chances de desenvolver crises asmáticas severas se expostas à fumaça do cigarro (Strachan e Cook, 1998). 

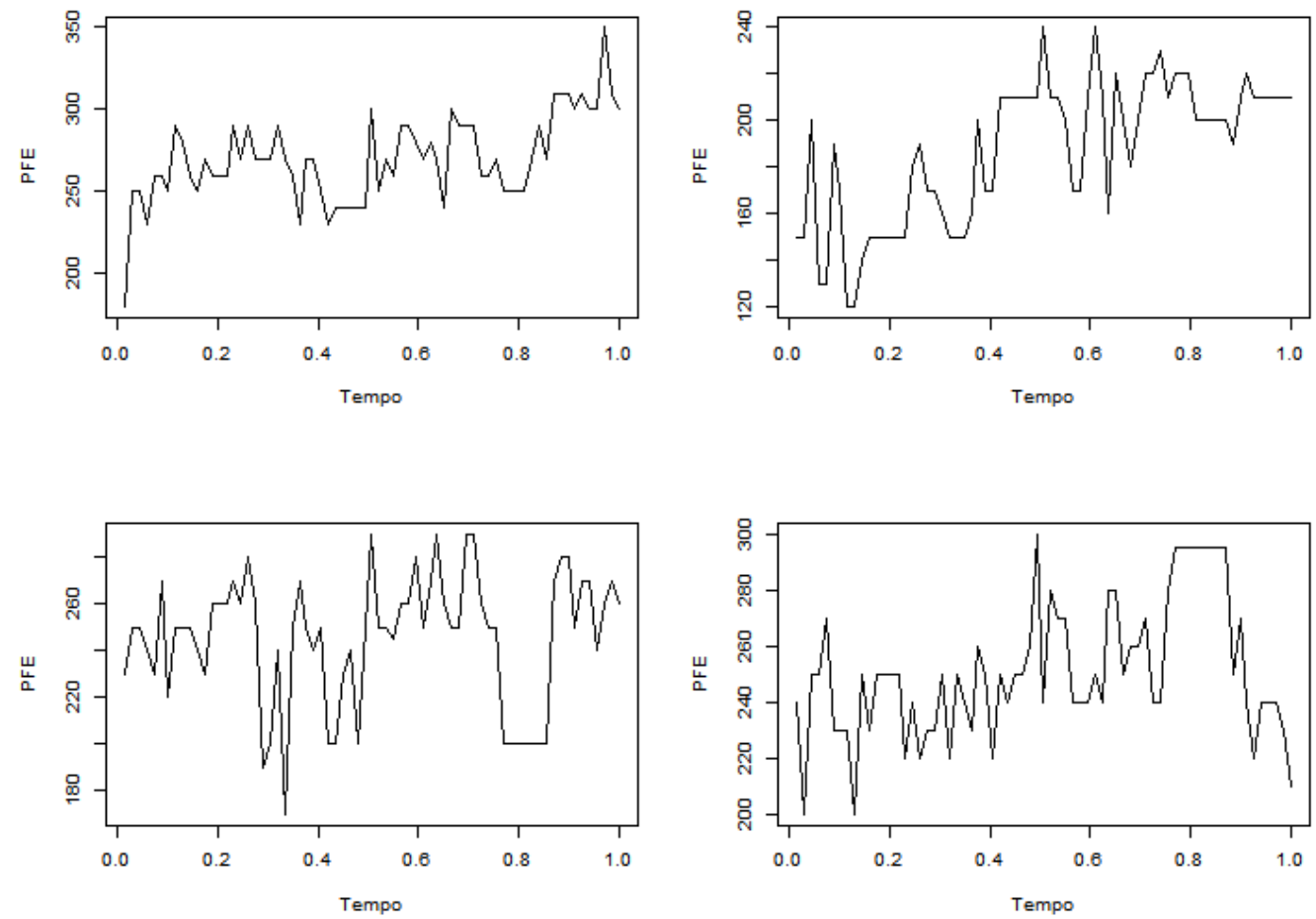

Figura 6.3: Amostra das séries dos indivíduos sem a condição de asma e expostos ao fumo domiciliar.
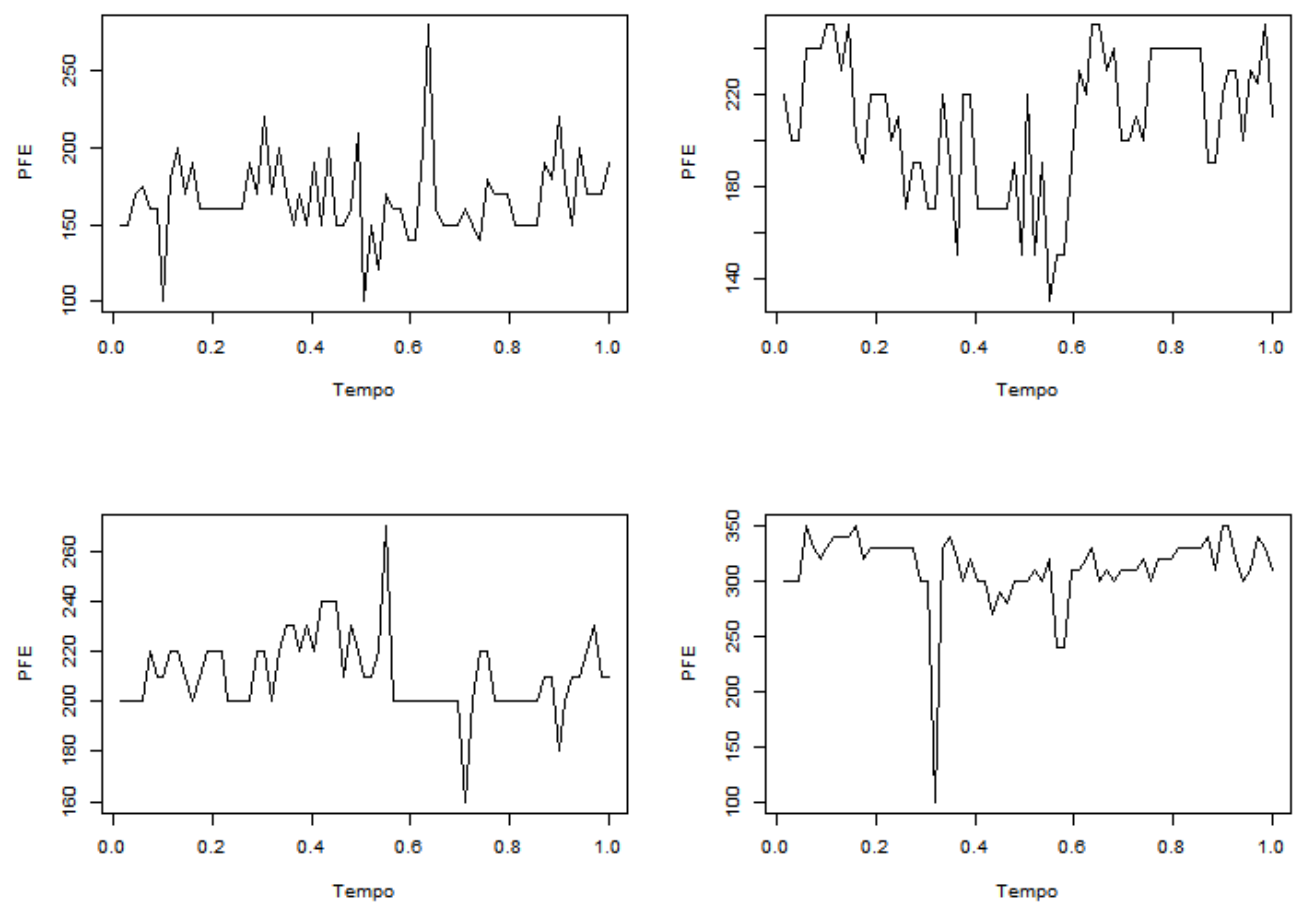

Figura 6.4: Amostra das séries indivíduos com a condição de asma e expostos ao fumo domiciliar. 


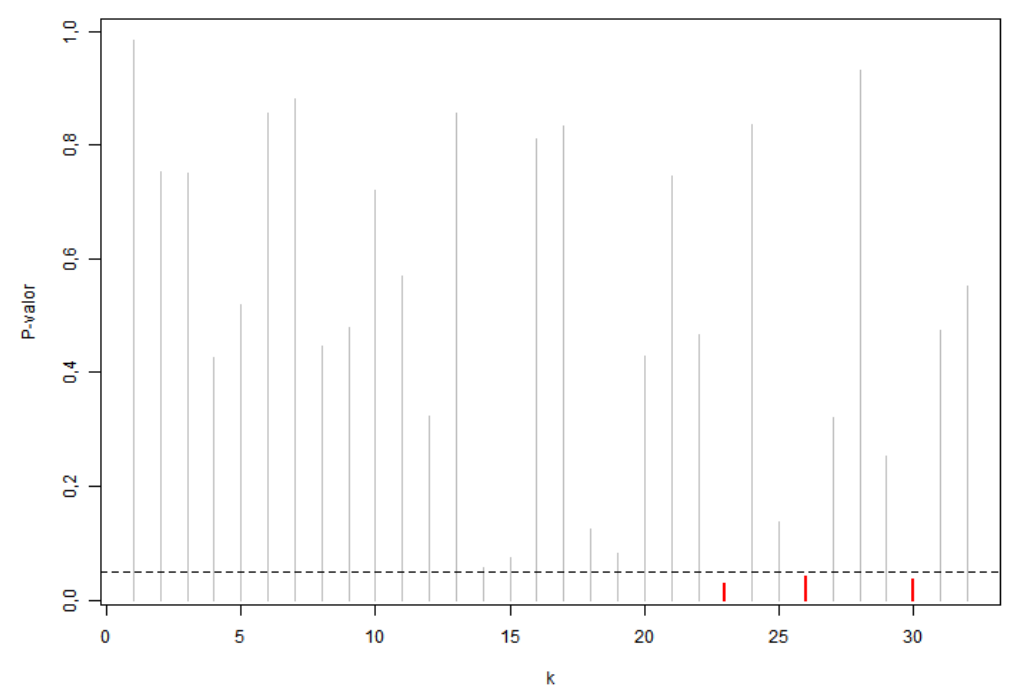

Figura 6.5: TDF: p-valores dos testes de interação para cada frequência $\lambda(k)$.

Como nosso principal objetivo é entender o efeito da asma na variável resposta, vamos estratificar a amostra em dois subgrupos, um apenas com os indivíduos expostos ao fumo e outro com os indivíduos não expostos, e então realizar a análise de variância em cada subgrupo separadamente. Para isso, vamos testar,

$$
\left\{\begin{array}{l}
H_{0}: \tau(\lambda)=0, \\
H_{1}: \tau(\lambda) \neq 0 .
\end{array}\right.
$$

Primeiramente, fixando os indivíduos expostos ao fumo domiciliar. Os resultados para as frequências $\lambda(k)=2 \pi k / T$ são apresentados na Figura 6.6 e na Tabela 6.2.

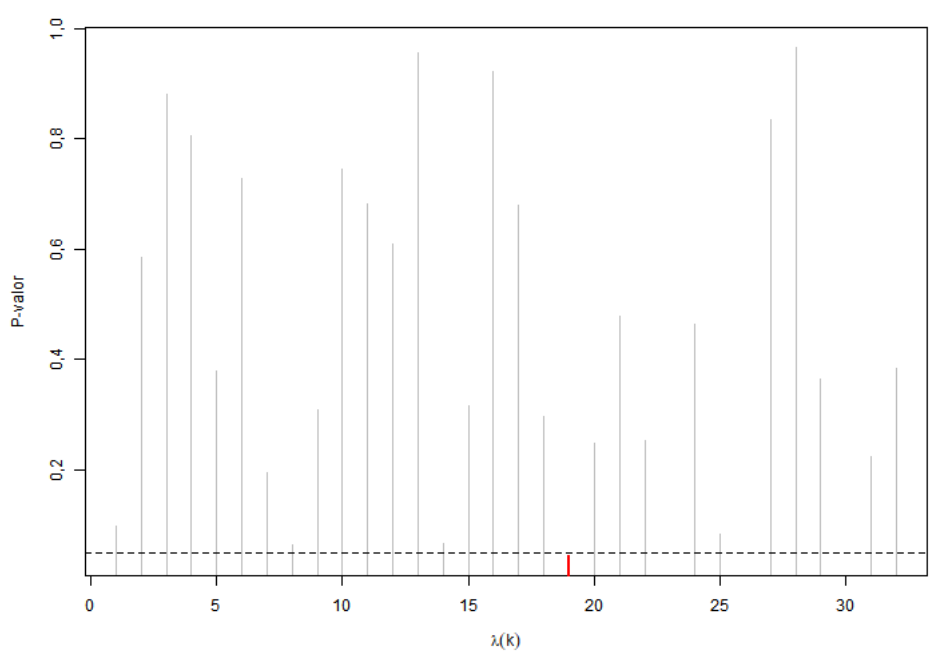

Figura 6.6: TDF: p-valores dos testes do efeito da asma nos expostos para cada frequência $\lambda(k)$.

Agora, fixando os indivíduos não expostos ao fumo domiciliar.Os p-valores dos testes do efeito da asma para as frequências $\lambda(k)=2 \pi k / T$ são apresentados na Figura 6.7 e na Tabela 6.3 . 
Tabela 6.2: TDF: p-valores dos testes do efeito da asma nos expostos para cada frequência $\lambda(k)$.

\begin{tabular}{c|rrrrrrrr}
\hline$\lambda$ & $0,00 \pi$ & $0,03 \pi$ & $0,06 \pi$ & $0,09 \pi$ & $0,12 \pi$ & $0,16 \pi$ & $0,19 \pi$ & $0,22 \pi$ \\
p-valor & 0,09 & 0,58 & 0,88 & 0,81 & 0,38 & 0,73 & 0,19 & 0,06 \\
\hline$\lambda$ & $0,25 \pi$ & $0,28 \pi$ & $0,31 \pi$ & $0,34 \pi$ & $0,38 \pi$ & $0,41 \pi$ & $0,44 \pi$ & $0,47 \pi$ \\
p-valor & 0,31 & 0,75 & 0,68 & 0,61 & 0,96 & 0,07 & 0,32 & 0,92 \\
\hline$\lambda$ & $0,50 \pi$ & $0,53 \pi$ & $0,56 \pi$ & $0,59 \pi$ & $0,62 \pi$ & $0,66 \pi$ & $0,69 \pi$ & $0,72 \pi$ \\
p-valor & 0,68 & 0,30 & $\mathbf{0 , 0 4}$ & 0,25 & 0,48 & 0,25 & - & 0,46 \\
\hline$\lambda$ & $0,75 \pi$ & $0,78 \pi$ & $0,81 \pi$ & $0,84 \pi$ & $0,88 \pi$ & $0,91 \pi$ & $0,94 \pi$ & $0,97 \pi$ \\
p-valor & 0,08 & - & 0,83 & 0,97 & 0,36 & - & 0,22 & 0,38 \\
\hline
\end{tabular}

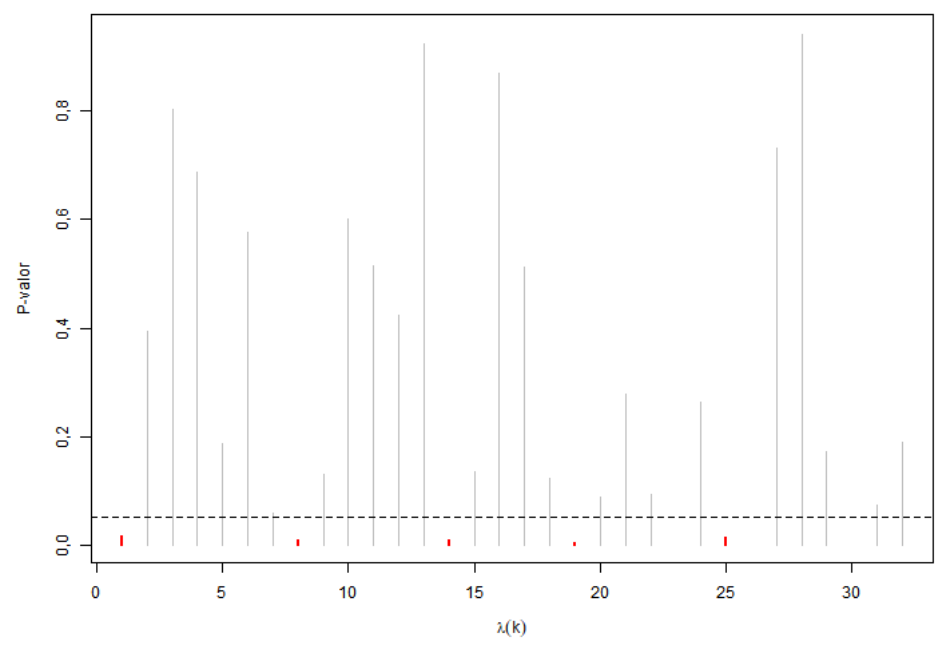

Figura 6.7: TDF: p-valores dos testes do efeito da asma nos não expostos para cada frequência $\lambda(k)$.

Tabela 6.3: TDF: p-valores dos testes do efeito da asma nos não expostos para cada frequência $\lambda(k)$.

\begin{tabular}{c|rrrrrrrr}
\hline$\lambda$ & $0,00 \pi$ & $0,03 \pi$ & $0,06 \pi$ & $0,09 \pi$ & $0,12 \pi$ & $0,16 \pi$ & $0,19 \pi$ & $0,22 \pi$ \\
p-valor & $\mathbf{0 , 0 2}$ & 0,39 & 0,80 & 0,69 & 0,19 & 0,58 & 0,06 & $\mathbf{0 , 0 1}$ \\
\hline$\lambda$ & $0,25 \pi$ & $0,28 \pi$ & $0,31 \pi$ & $0,34 \pi$ & $0,38 \pi$ & $0,41 \pi$ & $0,44 \pi$ & $0,47 \pi$ \\
p-valor & 0,13 & 0,60 & 0,51 & 0,42 & 0,93 & $\mathbf{0 , 0 1}$ & 0,14 & 0,87 \\
\hline$\lambda$ & $0,50 \pi$ & $0,53 \pi$ & $0,56 \pi$ & $0,59 \pi$ & $0,62 \pi$ & $0,66 \pi$ & $0,69 \pi$ & $0,72 \pi$ \\
p-valor & 0,51 & 0,12 & $\mathbf{0 , 0 1}$ & 0,09 & 0,28 & 0,09 & - & 026 \\
\hline$\lambda$ & $0,75 \pi$ & $0,78 \pi$ & $0,81 \pi$ & $0,84 \pi$ & $0,88 \pi$ & $0,91 \pi$ & $0,94 \pi$ & $0,97 \pi$ \\
p-valor & $\mathbf{0 , 0 1}$ & - & 0,73 & 0,94 & 0,17 & - & 0,91 & 0,58 \\
\hline
\end{tabular}

Pela Figura 6.7 e pela Tabela 6.3, nos testes para verificar o efeito da asma no PFE foram encontradas diferenças ao nível de significância de $5 \%$ em ambos os subgrupos. No grupo dos expostos ao fumo, apenas ocorreu diferença significativa na frequência $0,56 \pi$, indicando um efeito da asma a médio prazo. No grupo dos não expostos ao fumo, ocorreram diferenças significativas nas frequências $0 \pi, 0,22 \pi, 0,41 \pi, 0,56 \pi$ e $0,75 \pi$, indicando efeito da asma a curto, médio e longo prazo. A não exposição ao fumo tende a deixar mais evidente as diferenças entre o PFE dos asmáticos e não asmáticos. Por outro lado, a baixa quantidade de frequências com sinal significativo nos indivíduos expostos talvez se dê pelo fato da exposição ser tão prejudicial tanto para os asmáticos quanto para os não asmáticos. 
Por último, iremos testar o efeito de sinal em comum entre as séries. Foram criados quatro subgrupos: os asmáticos e expostos, não asmáticos e expostos, asmáticos e não expostos, e os não asmáticos e não expostos. Para isso, vamos testar a seguinte hipótese,

$$
\left\{\begin{array}{l}
H_{0}: \theta(\lambda)=0 \\
H_{1}: \theta(\lambda) \neq 0
\end{array}\right.
$$

Fixando os indivíduos asmáticos e expostos ao fumo domiciliar. Os p-valores dos testes do efeito de sinal comum para as frequências $\lambda(k)=2 \pi k / T$ são apresentados na Figura 6.8.

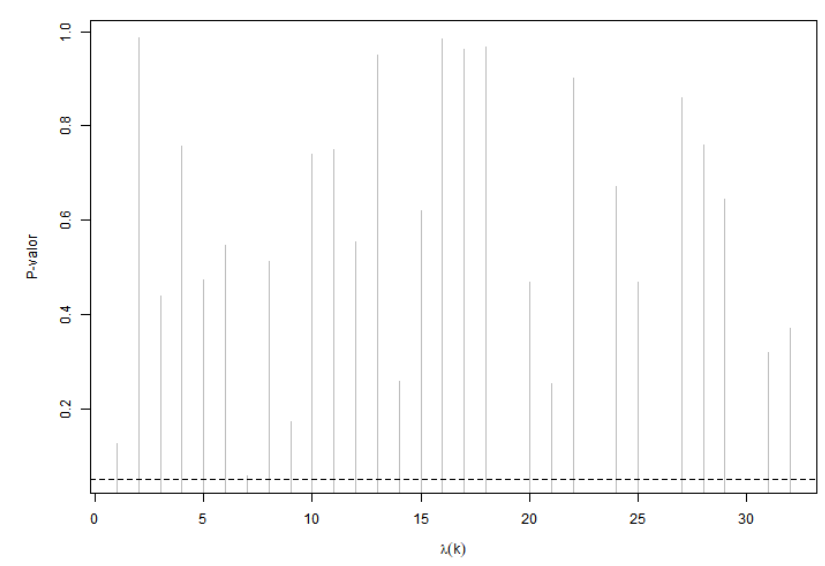

Figura 6.8: TDF: p-valores dos testes de sinal comum nos expostos e asmáticos para cada frequência $\lambda(k)$.

Fixando os indivíduos não asmáticos e expostos ao fumo domiciliar.Os p-valores dos testes do efeito de sinal comum para as frequências $\lambda(k)=2 \pi k / T$ são apresentados na Figura 6.9.

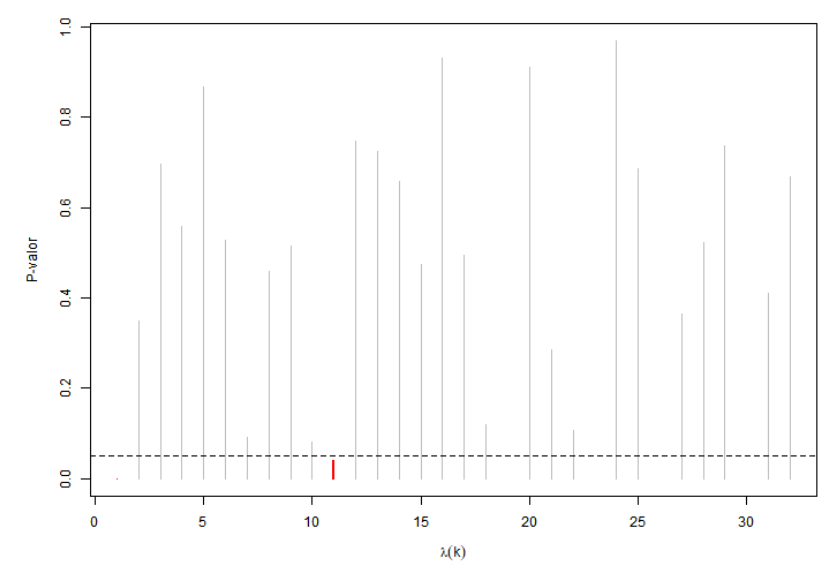

Figura 6.9: TDF: p-valores dos testes de sinal comum nos expostos e não asmáticos para cada frequência $\lambda(k)$.

Fixando os indivíduos asmáticos e não expostos ao fumo domiciliar. Os p-valores dos testes do efeito de sinal comum para as frequências $\lambda(k)=2 \pi k / T$ são apresentados na Figura 6.10. 


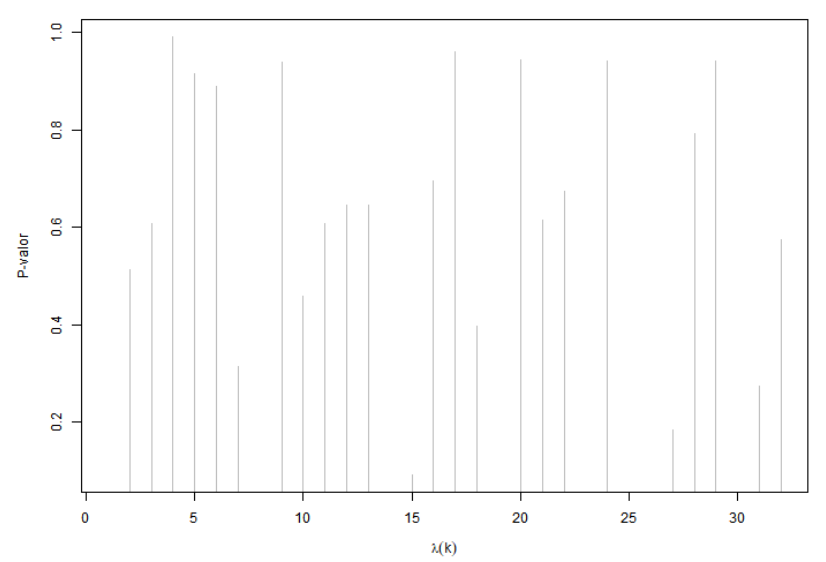

Figura 6.10: TDF: p-valores dos testes de sinal comum nos não expostos e asmáticos para cada frequência $\lambda(k)$.

Fixando os indivíduos não asmáticos e não expostos ao fumo domiciliar. Os p-valores dos testes do efeito de sinal comum para as frequências $\lambda(k)=2 \pi k / T$ são apresentados na Figura 6.11.

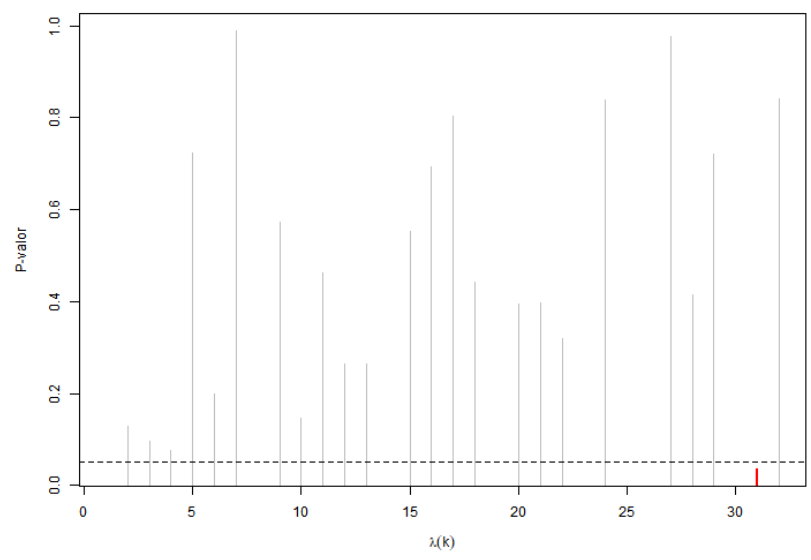

Figura 6.11: TDF: p-valores dos testes de sinal comum nos não expostos e não asmáticos para cada frequência $\lambda(k)$.

Nos testes para verificar a existência sinal em comum no PFE foram encontrados sinais em comum, ao nível de significância de 5\%, em dois dos quatro subgrupos, nos grupos do não asmáticos e expostos e no grupo dos não asmáticos e não expostos.

Devemos ter um pouco de cautela com os resultados obtidos pelo método de análise de variância usando a transformada discreta de Fourier, pois a efetividade desse método depende fortemente da estacionariedade das observações. Por isso, não podemos ter certeza que o método foi totalmente eficiente. A abordagem por ondaletas, que será utilizada a seguir, tende a ser mais robusta nesse sentido, pois abrimos mão da hipótese de estacionariedade. 


\subsection{Análise de variância utilizando ondaletas}

Para a aplicação da ANOVA no contexto de ondaletas, iremos abordar o problema por meio de duas formas, utilizando a transformada discreta de ondaletas (TDO) e a transformada discreta de ondaletas não decimada (TDOND). A aplicação será feita por meio de duas famílias de ondaletas: Extremal Phase D8 e a Least Asymmetric LA8

Considerando a asma, fumo domiciliar e a interação entre eles, temos o seguinte modelo de análise de variância.

$Y_{\ell m s}(t)=\mu_{\ell m s}+\Omega(t)+\Gamma(t) \times I\left(\right.$ Asma $\left._{\ell m s}\right)+\Theta(t) \times I\left(F u m o_{\ell m s}\right)+\Lambda(t) \times I\left(A s m a_{\ell m s}\right) I\left(F u m o_{\ell m s}\right)+\epsilon_{\ell m s}(t)$,

em que $\ell=1, \ldots, L_{m s}, m=1,2, s=1,2, t=0, \ldots, T-1$, de tal forma que $Y_{\ell m s}(t)$ é o pico de fluxo expiratório da $\ell$-ésima unidade amostral dentro dos níveis $m$ e $s$ no instante $t$, $\mu_{\ell m s}$ é o nível da série, $\Omega(t)$ é o sinal em comum entre as séries, $\Gamma(t)$ é o efeito da asma no instante $t, \Theta(t)$ é o efeito do fumo domiciliar no instante $t, \Lambda(t)$ é o efeito da interação entre asma e fumo domiciliar no instante $t, \epsilon_{\ell m s}(t)$ é um termo de erro aleatório.

\subsubsection{Transformada discreta de ondaletas}

No caso da transformada discreta de ondaletas, o modelo pode ser reescrito da seguinte maneira:

$w_{\ell m s}(j, k)=\theta(j, k)+\tau(j, k) \times I\left(A_{s m a_{\ell m s}}\right)+\eta(j, k) \times I\left(F_{u m o_{\ell m s}}\right)+\delta(j, k) \times I\left(A s m a_{\ell m s}\right) I\left(F u m o_{\ell m s}\right)+\varepsilon_{\ell m s}(j, k)$

em que $w_{\ell m s}(j, k)$ é a transformada do pico de fluxo expiratório da $\ell$-ésima unidade amostral dentro dos níveis $m$ e $s$ nos índices $(j, k), \theta(j, k)$ é o sinal em comum nos índices $(j, k), \tau$ é o efeito da asma nos índices $(j, k), \eta(j, k)$ é o efeito do fumo domiciliar nos índices $(j, k)$, $\delta(j, k)$ é o efeito da interação entre asma e fumo domiciliar nos índices $(j, k)$. Além disso, $\varepsilon_{\ell m s}(j, k)$ é um termo de erro aleatório.

Primeiramente, iremos testar se o efeito de interação entre o fumo e a asma são significativos ou não,

$$
\left\{\begin{array}{l}
H_{0}: \delta(j, k)=0 \\
H_{1}: \delta(j, k) \neq 0
\end{array}\right.
$$

Os resultados para os índices $(j, k)$ são apresentados na Figura 6.12 e na Tabela 6.4 .

Pelas Figura 6.12 e Tabela 6.4, podemos observar que ao nível de significância de 5\%, foram significativos no nível $j=5$ em ambas as ondaletas e $j=4$ na ondaleta Least Asymmetric, sugerindo que a interação entre exposição à fumaça no ambiente e a condição de asma causa diferenças no PFE a curto prazo aos indivíduos expostos. Essas evidências convergem com os resultados obtidos por meio da TDF, e novamente corroboram a literatura sobre o risco da interação entre exposição ao fumo e asma. Os índices e níveis com diferenças significavas não serão utilizados para verificar a diferença de efeito da asma.

Assim como foi feito caso da transformada de Fourier, vamos estratificar a amostra em dois subgrupos: expostos ao fumo e não expostos ao fumo e então aplicar a ANOVA para verificar o efeito da asma. Para isso, vamos testar,

$$
\left\{\begin{array}{l}
H_{0}: \tau(j, k)=0 \\
H_{1}: \tau(j, k) \neq 0
\end{array}\right.
$$



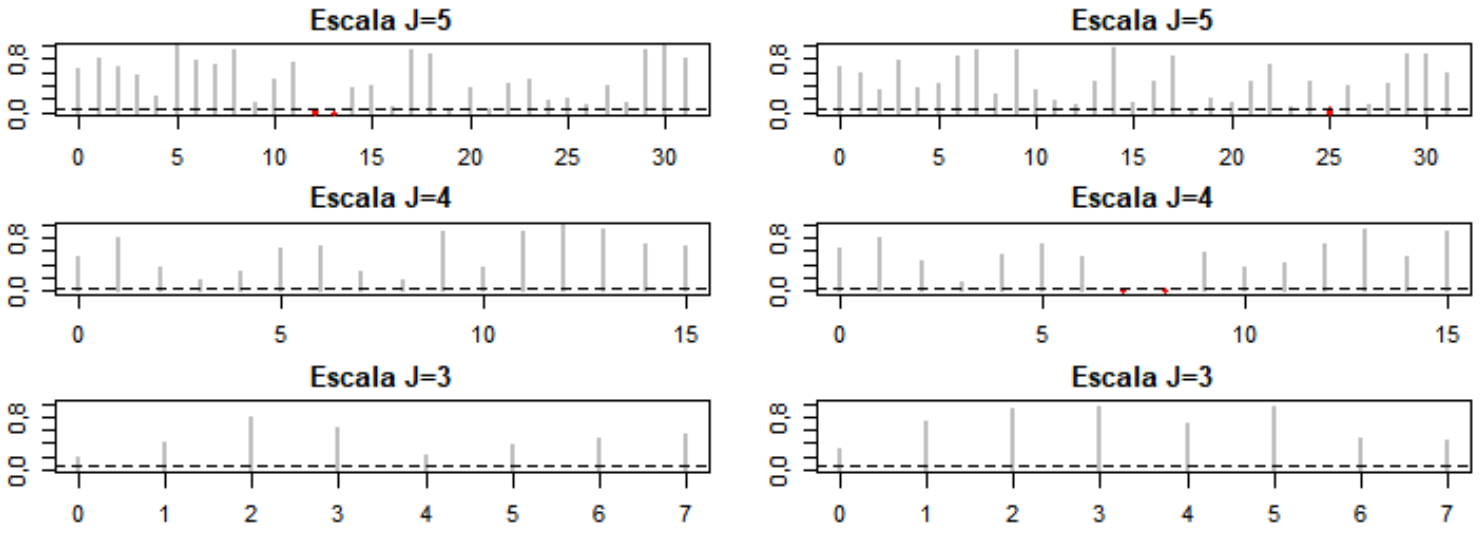

Escala $\mathrm{J}=\mathbf{2}$
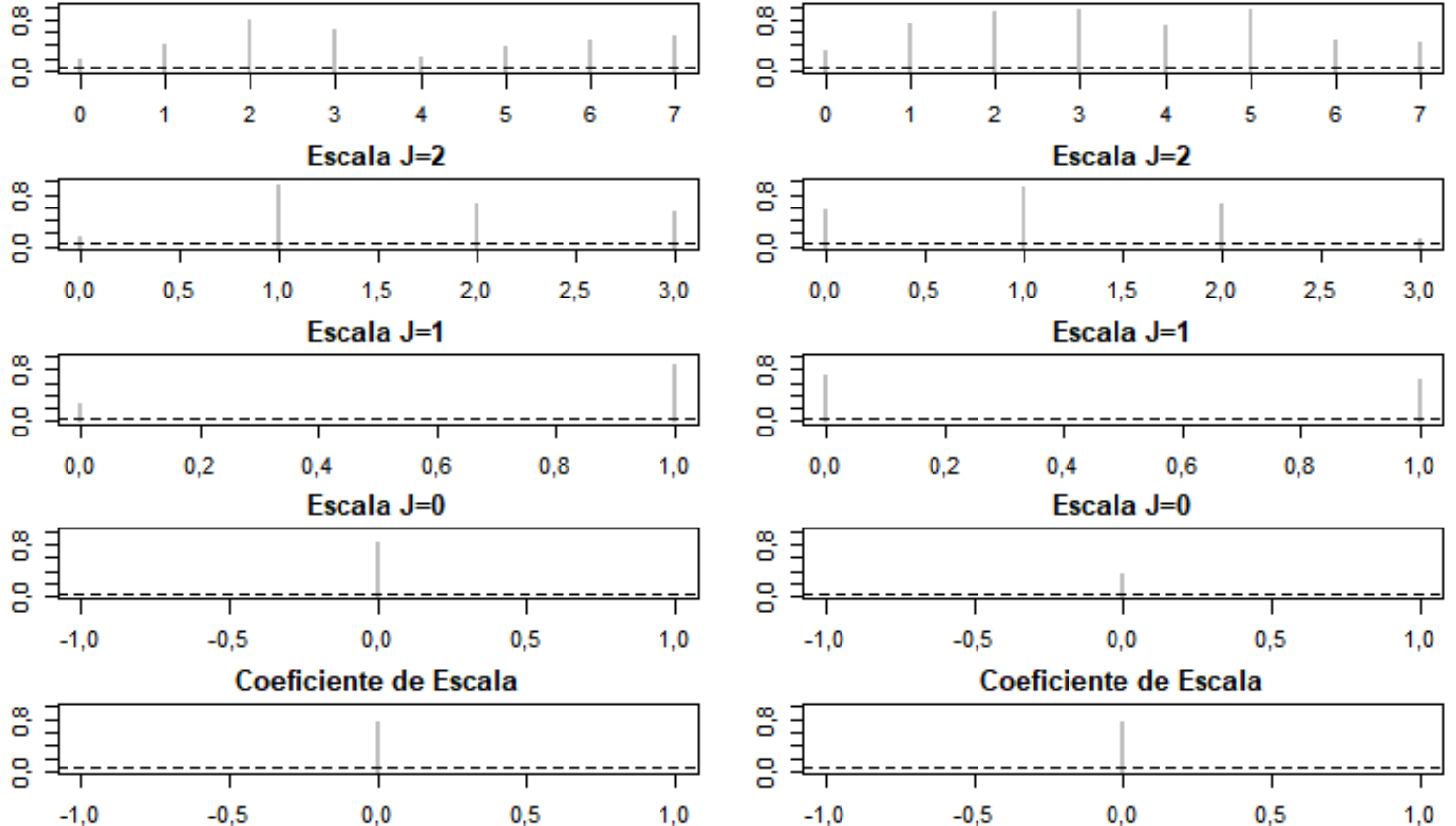

Figura 6.12: TDO: p-valores dos testes de interação para cada indice $(j, k)$. Extremal Phase (D8) a esquerda e Least Asymmetric (LA8) a direita.

Tabela 6.4: TDO: p-valores dos testes de interação para cada indice $(j, k)$, indices significativos estão em destaque.

\begin{tabular}{|c|c|c|c|c|c|c|c|c|c|c|c|c|c|c|c|c|}
\hline & \multicolumn{8}{|c|}{ Extremal Phase (D8) } & \multicolumn{8}{|c|}{ Least Asymmetric (LA8) } \\
\hline$(\mathbf{j}, \mathbf{k})$ & $(5,0)$ & $(5,1)$ & $(5,2)$ & $(5,3)$ & $(5,4)$ & $(5,5)$ & $(5,6)$ & $(5,7)$ & $(5,0)$ & $(5,1)$ & $(5,2)$ & $(5,3)$ & $(5,4)$ & $(5,5)$ & $(5,6)$ & $(5,7)$ \\
\hline p-valor & 0,66 & 0,80 & 0,69 & 0,57 & 0,25 & 0,99 & 0,80 & 0,71 & 0,70 & 0,59 & 0,35 & 0,77 & 0,36 & 0,43 & 0,85 & 0,95 \\
\hline$(\mathbf{j}, \mathbf{k})$ & $(5,8)$ & $(5,9)$ & $(5,10)$ & $(5,11)$ & $(5,12)$ & $(5,13)$ & $(5,14)$ & $(5,15)$ & $(5,8)$ & $(5,9)$ & $(5,10)$ & $(5,11)$ & $(5,12)$ & $(5,13)$ & $(5,14)$ & $(5,15)$ \\
\hline p-valor & 0,95 & 0,14 & 0,50 & 0,75 & 0,01 & 0,01 & 0,36 & 0,38 & 0,27 & 0,93 & 0,34 & 0,18 & 0,11 & 0,46 & 0,96 & 0,16 \\
\hline$(\mathbf{j}, \mathbf{k})$ & $(5,16)$ & $(5,17)$ & $(5,18)$ & $(5,19)$ & $(5,20)$ & $(5,21)$ & $(5,22)$ & $(5,23)$ & $(5,16)$ & $(5,17)$ & $(5,18)$ & $(5,19)$ & $(5,20)$ & $(5,21)$ & $(5,22)$ & $(5,23)$ \\
\hline p-valor & 0,09 & 0,95 & 0,88 & 0,05 & 0,36 & 0,05 & 0,45 & 0,50 & 0,47 & 0,85 & 0,06 & 0,21 & 0,13 & 0,47 & 0,73 & 0,09 \\
\hline$(\mathbf{j}, \mathbf{k})$ & $(5,24)$ & $(5,25)$ & $(5,26)$ & $(5,27)$ & $(5,28)$ & $(5,29)$ & $(5,30)$ & $(5,31)$ & $(5,24)$ & $(5,25)$ & $(5,26)$ & $(5,27)$ & $(5,28)$ & $(5,29)$ & $(5,30)$ & $(5,31)$ \\
\hline p-valor & 0,17 & 0,20 & 0,12 & 0,41 & 0,14 & 0,94 & 1,00 & 0,81 & 0,46 & 0,04 & 0,41 & 0,12 & 0,42 & 0,88 & 0,86 & 0,60 \\
\hline$(\mathbf{j}, \mathbf{k})$ & $(4,0)$ & $(4,1)$ & $(4,2)$ & $(4,3)$ & $(4,4)$ & $(4,5)$ & $(4,6)$ & $(4,7)$ & $(4,0)$ & $(4,1)$ & $(4,2)$ & $(4,3)$ & $(4,4)$ & $(4,5)$ & $(4,6)$ & $(4,7)$ \\
\hline p-valor & 0,52 & 0,79 & 0,37 & 0,16 & 0,29 & 0,65 & 0,69 & 0,28 & 0,64 & 0,82 & 0,45 & 0,14 & 0,54 & 0,71 & 0,53 & 0,02 \\
\hline$(\mathbf{j}, \mathbf{k})$ & $(4,8)$ & $(4,9)$ & $(4,10)$ & $(4,11)$ & $(4,12)$ & $(4,13)$ & $(4,14)$ & $(4,15)$ & $(4,8)$ & $(4,9)$ & $(4,10)$ & $(4,11)$ & $(4,12)$ & $(4,13)$ & $(4,14)$ & $(4,15)$ \\
\hline p-valor & 0,17 & 0,89 & 0,35 & 0,89 & 0,99 & 0,93 & 0,72 & 0,68 & 0,01 & 0,59 & 0,36 & 0,44 & 0,71 & 0,92 & 0,53 & 0,90 \\
\hline$(\mathbf{j}, \mathbf{k})$ & $(3,0)$ & $(3,1)$ & $(3,2)$ & $(3,3)$ & $(3,4)$ & $(3,5)$ & $(3,6)$ & $(3,7)$ & $(3,0)$ & $(3,1)$ & $(3,2)$ & $(3,3)$ & $(3,4)$ & $(3,5)$ & $(3,6)$ & $(3,7)$ \\
\hline p-valor & 0,21 & 0,40 & 0,79 & 0,65 & 0,21 & 0,40 & 0,46 & 0,53 & 0,32 & 0,74 & 0,92 & 0,95 & 0,71 & 0,95 & 0,49 & 0,43 \\
\hline$(\mathbf{j}, \mathbf{k})$ & $(2$, & & $(2$, & & $(2$, & & $(2$, & & $(2$ & & $(2$, & & $(2$, & & $(2$, & \\
\hline p-valor & 0 , & & 0, & & 0, & & 0 & & 0 , & & 0, & & 0 , & & 0 , & \\
\hline$(\mathbf{j}, \mathbf{k})$ & & $(1$, & & & & $(1$, & & & & $(1$, & & & & $(1$ & & \\
\hline p-valor & & 0 & & & & 0, & & & & 0 & & & & 0 , & & \\
\hline$(\mathbf{j}, \mathbf{k})$ & & & & $(0$, & & & & & & & & $(0$ & & & & \\
\hline p-valor & & & & 0 & & & & & & & & 0 , & & & & \\
\hline $\begin{array}{c}\text { Coef. Escala } \\
\text { p-valor }\end{array}$ & & & & $\begin{array}{l}(0, \\
0\end{array}$ & & & & & & & & $\begin{array}{l}(0 \\
0\end{array}$ & & & & \\
\hline
\end{tabular}



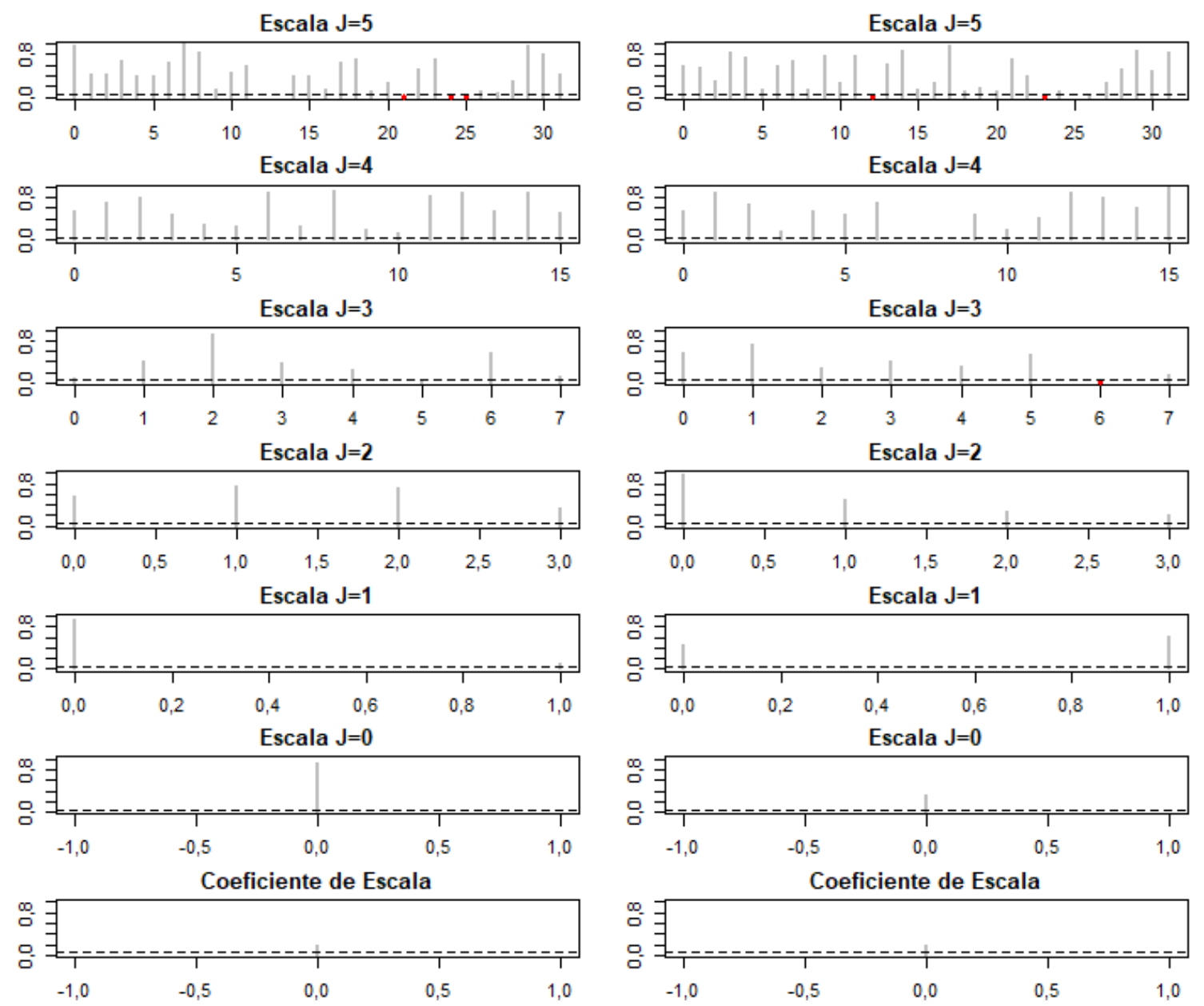

Figura 6.13: TDO: p-valores dos testes do efeito da asma nos expostos para cada indice $(j, k)$. Extremal Phase (D8) a esquerda e Least Asymmetric (LA8) a direita.

Tabela 6.5: TDO: p-valores dos testes do efeito da asma nos expostos para cada índice $(j, k)$.

\begin{tabular}{|c|c|c|c|c|c|c|c|c|c|c|c|c|c|c|c|c|}
\hline & \multicolumn{8}{|c|}{ Extremal Phase (D8) } & \multicolumn{8}{|c|}{ Least Asymmetric (LA8) } \\
\hline$(\mathbf{j}, \mathbf{k})$ & $(5,0)$ & $(5,1)$ & $(5,2)$ & $(5,3)$ & $(5,4)$ & $(5,5)$ & $(5,6)$ & $(5,7)$ & $(5,0)$ & $(5,1)$ & $(5,2)$ & $(5,3)$ & $(5,4)$ & $(5,5)$ & $(5,6)$ & $(5,7)$ \\
\hline p-valor & 0,96 & 0,43 & 0,43 & 0,69 & 0,41 & 0,40 & 0,65 & 0,99 & 0,60 & 0,57 & 0,31 & 0,84 & 0,74 & 0,15 & 0,60 & 0,67 \\
\hline$(\mathbf{j}, \mathbf{k})$ & $(5,8)$ & $(5,9)$ & $(5,10)$ & $(5,11)$ & $(5,12)$ & $(5,13)$ & $(5,14)$ & $(5,15)$ & $(5,8)$ & $(5,9)$ & $(5,10)$ & $(5,11)$ & $(5,12)$ & $(5,13)$ & $(5,14)$ & $(5,15)$ \\
\hline p-valor & 0,85 & 0,14 & 0,45 & 0,59 & - & - & 0,41 & 0,41 & 0,14 & 0,78 & 0,27 & 0,78 & 0,03 & 0,62 & 0,88 & 0,14 \\
\hline$(\mathbf{j}, \mathbf{k})$ & $(5,16)$ & $(5,17)$ & $(5,18)$ & $(5,19)$ & $(5,20)$ & $(5,21)$ & $(5,22)$ & $(5,23)$ & $(5,16)$ & $(5,17)$ & $(5,18)$ & $(5,19)$ & $(5,20)$ & $(5,21)$ & $(5,22)$ & $(5,23)$ \\
\hline p-valor & 0,16 & 0,66 & 0,71 & 0,11 & 0,28 & 0,02 & 0,51 & 0,71 & 0,26 & 0,97 & 0,10 & 0,18 & 0,10 & 0,72 & 0,39 & 0,01 \\
\hline$(\mathbf{j}, \mathbf{k})$ & $(5,24)$ & $(5,25)$ & $(5,26)$ & $(5,27)$ & $(5,28)$ & $(5,29)$ & $(5,30)$ & $(5,31)$ & $(5,24)$ & $(5,25)$ & $(5,26)$ & $(5,27)$ & $(5,28)$ & $(5,29)$ & $(5,30)$ & $(5,31)$ \\
\hline p-valor & 0,01 & 0,01 & 0,11 & 0,08 & 0,30 & 0,96 & 0,81 & 0,44 & 0,12 & & 0,06 & 0,26 & 0,54 & 0,86 & 0,50 & 0,86 \\
\hline$(\mathbf{j}, \mathbf{k})$ & $(4,0)$ & $(4,1)$ & $(4,2)$ & $(4,3)$ & $(4,4)$ & $(4,5)$ & $(4,6)$ & $(4,7)$ & $(4,0)$ & $(4,1)$ & $(4,2)$ & $(4,3)$ & $(4,4)$ & $(4,5)$ & $(4,6)$ & $(4,7)$ \\
\hline p-valor & 0,55 & 0,69 & 0,80 & 0,49 & 0,30 & 0,27 & 0,88 & 0,27 & 0,54 & 0,90 & 0,67 & 0,17 & 0,54 & 0,49 & 0,70 & - \\
\hline$(\mathbf{j}, \mathbf{k})$ & $(4,8)$ & $(4,9)$ & $(4,10)$ & $(4,11)$ & $(4,12)$ & $(4,13)$ & $(4,14)$ & $(4,15)$ & $(4,8)$ & $(4,9)$ & $(4,10)$ & $(4,11)$ & $(4,12)$ & $(4,13)$ & $(4,14)$ & $(4,15)$ \\
\hline p-valor & 0,92 & 0,20 & 0,13 & 0,83 & 0,91 & 0,56 & 0,89 & 0,53 & - & 0,47 & 0,22 & 0,43 & 0,89 & 0,80 & 0,60 & 0,99 \\
\hline$(\mathbf{j}, \mathbf{k})$ & $(3,0)$ & $(3,1)$ & $(3,2)$ & $(3,3)$ & $(3,4)$ & $(3,5)$ & $(3,6)$ & $(3,7)$ & $(3,0)$ & $(3,1)$ & $(3,2)$ & $(3,3)$ & $(3,4)$ & $(3,5)$ & $(3,6)$ & $(3,7)$ \\
\hline p-valor & 0,11 & 0,42 & 0,91 & 0,37 & 0,25 & 0,07 & 0,57 & 0,13 & 0,57 & 0,75 & 0,29 & 0,41 & 0,32 & 0,54 & 0,02 & 0,16 \\
\hline$(\mathbf{j}, \mathbf{k})$ & $(2$, & & $(2$, & & $(2$, & & $(2$, & & $(2$, & & $(2$, & & $(2$, & & $(2$, & \\
\hline p-valor & 0 , & & 0 & & 0,7 & & 0,3 & & 0,0 & & 0, & & 0,2 & & 0 & \\
\hline$(\mathbf{j}, \mathbf{k})$ & \multirow{2}{*}{\multicolumn{4}{|c|}{$\begin{array}{c}(1,0) \\
0,95\end{array}$}} & \multirow{2}{*}{\multicolumn{4}{|c|}{$\begin{array}{c}(1,1) \\
0,12\end{array}$}} & \multirow{2}{*}{\multicolumn{4}{|c|}{$\begin{array}{l}(1,0) \\
0,47\end{array}$}} & \multirow{2}{*}{\multicolumn{4}{|c|}{$\begin{array}{c}(1,1) \\
0,62\end{array}$}} \\
\hline p-valor & & & & & & & & & & & & & & & & \\
\hline$(\mathbf{j}, \mathbf{k})$ & \multirow{2}{*}{\multicolumn{8}{|c|}{$\begin{array}{c}(0,0) \\
0,92\end{array}$}} & \multirow{2}{*}{\multicolumn{8}{|c|}{$\begin{array}{c}(0,0) \\
0,33\end{array}$}} \\
\hline p-valor & & & & & & & & & & & & & & & & \\
\hline Coef. Escala & \multirow{2}{*}{\multicolumn{8}{|c|}{$\begin{array}{c}(0,0) \\
0,19\end{array}$}} & \multirow{2}{*}{\multicolumn{8}{|c|}{$\begin{array}{c}(0,0) \\
0,19\end{array}$}} \\
\hline p-valor & & & & & & & & & & & & & & & & \\
\hline
\end{tabular}


Primeiramente, fixando os indivíduos expostos ao fumo domiciliar, os resultados dos testes se encontram na 6.13 e na Tabela 6.5 .

Agora, fixando os indivíduos não expostos ao fumo domiciliar, os resultados dos testes se encontram na 6.14 e na Tabela 6.6.
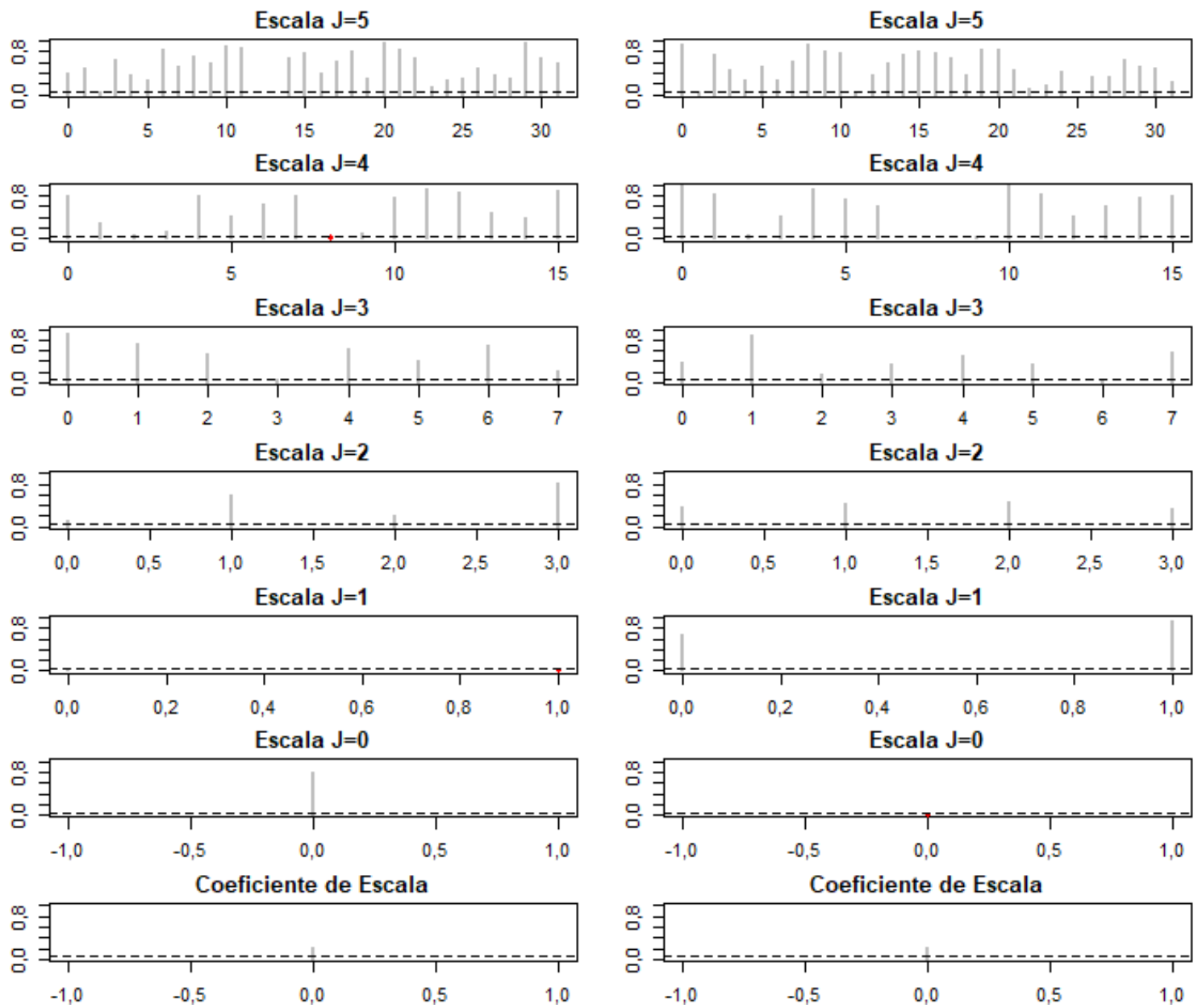

Figura 6.14: TDO: p-valores dos testes do efeito da asma nos não expostos para cada índice $(j, k)$. Extremal Phase (D8) a esquerda e Least Asymmetric (LA8) a direita.

Nos testes para verificar o efeito da asma no PFE foram encontradas diferenças ao nível de significância de $5 \%$ em ambos os subgrupos. No grupo dos expostos ao fumo domiciliar foram encontrados efeitos significativos no nível $J=5 \mathrm{em}$ ambas as ondaletas e no nível $J=3$ para a ondaleta Least Asymmetric, sugerindo efeito de curto e médio prazo do efeito da asma no PFE. No grupo dos não expostos foram encontrados efeitos significativos nos níveis $J=4$ e $J=1$ para a ondaleta Extremal Phase e no nível $J=0$ para a ondaleta Least Asymmetric, sugerindo efeitos de curto e longo prazo do efeito da asma no PFE. Novamente os resultados obtidos convergem com os obtidos pela TDF.

Por último iremos testar o efeito de sinal em comum entre as séries. Foram criados quatro subgrupos: os asmáticos e expostos, não asmáticos e expostos, asmáticos e não expostos e os não asmáticos e não expostos. Para isso, vamos testar a seguinte hipótese,

$$
\left\{\begin{array}{l}
H_{0}: \theta(j, k)=0 \\
H_{1}: \theta(j, k) \neq 0
\end{array}\right.
$$

Fixando os indivíduos asmáticos e expostos ao fumo domiciliar os resultados dos testes se encontram na Figura 6.15. 
Tabela 6.6: TDO: p-valores dos testes do efeito da asma nos não expostos para cada índice $(j, k)$.

\begin{tabular}{|c|c|c|c|c|c|c|c|c|c|c|c|c|c|c|c|c|}
\hline & \multicolumn{8}{|c|}{ Extremal Phase (D8) } & \multicolumn{8}{|c|}{ Least Asymmetric (LA8) } \\
\hline$(\mathbf{j}, \mathbf{k})$ & $(5,0)$ & $(5,1)$ & $(5,2)$ & $(5,3)$ & $(5,4)$ & $(5,5)$ & $(5,6)$ & $(5,7)$ & $(5,0)$ & $(5,1)$ & $(5,2)$ & $(5,3)$ & $(5,4)$ & $(5,5)$ & $(5,6)$ & $(5,7)$ \\
\hline p-valor & 0,40 & 0,51 & 0,06 & 0,65 & 0,38 & 0,26 & 0,83 & 0,54 & 0,95 & 0,06 & 0,73 & 0,48 & 0,28 & 0,54 & 0,27 & 0,61 \\
\hline$(\mathbf{j}, \mathbf{k})$ & $(5,8)$ & $(5,9)$ & $(5,10)$ & $(5,11)$ & $(5,12)$ & $(5,13)$ & $(5,14)$ & $(5,15)$ & $(5,8)$ & $(5,9)$ & $(5,10)$ & $(5,11)$ & $(5,12)$ & $(5,13)$ & $(5,14)$ & $(5,15)$ \\
\hline p-valor & 0,72 & 0,58 & 0,92 & 0,89 & - & - & 0,69 & 0,77 & 0,94 & 0,80 & 0,78 & 0,06 & 0,36 & 0,58 & 0,74 & 0,82 \\
\hline$(\mathbf{j}, \mathbf{k})$ & $(5,16)$ & $(5,17)$ & $(5,18)$ & $(5,19)$ & $(5,20)$ & $(5,21)$ & $(5,22)$ & $(5,23)$ & $(5,16)$ & $(5,17)$ & $(5,18)$ & $(5,19)$ & $(5,20)$ & $(5,21)$ & $(5,22)$ & $(5,23)$ \\
\hline p-valor & 0,41 & 0,61 & 0,82 & 0,32 & 0,96 & 0,85 & 0,70 & 0,14 & 0,78 & 0,70 & 0,38 & 0,86 & 0,84 & 0,46 & 0,11 & 0,19 \\
\hline$(\mathbf{j}, \mathbf{k})$ & $(5,24)$ & $(5,25)$ & $(5,26)$ & $(5,27)$ & $(5,28)$ & $(5,29)$ & $(5,30)$ & $(5,31)$ & $(5,24)$ & $(5,25)$ & $(5,26)$ & $(5,27)$ & $(5,28)$ & $(5,29)$ & $(5,30)$ & $(5,31)$ \\
\hline p-valor & 0,26 & 0,31 & 0,51 & 0,38 & 0,31 & 0,96 & 0,67 & 0,58 & 0,42 & - & 0,34 & 0,33 & 0,66 & 0,54 & 0,51 & 0,25 \\
\hline$(\mathbf{j}, \mathbf{k})$ & $(4,0)$ & $(4,1)$ & $(4,2)$ & $(4,3)$ & $(4,4)$ & $(4,5)$ & $(4,6)$ & $(4,7)$ & $(4,0)$ & $(4,1)$ & $(4,2)$ & $(4,3)$ & $(4,4)$ & $(4,5)$ & $(4,6)$ & $(4,7)$ \\
\hline p-valor & 0,79 & 0,31 & 0,06 & 0,15 & 0,81 & 0,43 & 0,63 & 0,79 & 0,99 & 0,82 & 0,07 & 0,42 & 0,93 & 0,75 & 0,60 & - \\
\hline$(\mathbf{j}, \mathbf{k})$ & $(4,8)$ & $(4,9)$ & $(4,10)$ & $(4,11)$ & $(4,12)$ & $(4,13)$ & $(4,14)$ & $(4,15)$ & $(4,8)$ & $(4,9)$ & $(4,10)$ & $(4,11)$ & $(4,12)$ & $(4,13)$ & $(4,14)$ & $(4,15)$ \\
\hline p-valor & 0,03 & 0,10 & 0,76 & 0,94 & 0,88 & 0,50 & 0,38 & 0,90 & & 0,06 & 0,99 & 0,82 & 0,41 & 0,62 & 0,78 & 0,81 \\
\hline$(\mathbf{j}, \mathbf{k})$ & $(3,0)$ & $(3,1)$ & $(3,2)$ & $(3,3)$ & $(3,4)$ & $(3,5)$ & $(3,6)$ & $(3,7)$ & $(3,0)$ & $(3,1)$ & $(3,2)$ & $(3,3)$ & $(3,4)$ & $(3,5)$ & $(3,6)$ & $(3,7)$ \\
\hline p-valor & 0,92 & 0,74 & 0,55 & 0,06 & 0,63 & 0,42 & 0,70 & 0,23 & 0,37 & 0,90 & 0,16 & 0,34 & 0,52 & 0,34 & 0,06 & 0,59 \\
\hline$(\mathbf{j}, \mathbf{k})$ & & & $(2$, & & $(2$, & & $(2$, & & $(2$, & & $(2$, & & $(2$, & & $(2$, & \\
\hline p-valor & & & 0 , & & 0,2 & & 0,8 & & 0, & & 0,4 & & 0,4 & & 0 & \\
\hline$(\mathbf{j}, \mathbf{k})$ & \multirow{2}{*}{\multicolumn{4}{|c|}{$\begin{array}{c}(1,0) \\
0,06\end{array}$}} & \multirow{2}{*}{\multicolumn{4}{|c|}{$\begin{array}{l}(1,1) \\
\mathbf{0 , 0 2}\end{array}$}} & \multirow{2}{*}{\multicolumn{4}{|c|}{$\begin{array}{l}(1,0) \\
0,67\end{array}$}} & \multirow{2}{*}{\multicolumn{4}{|c|}{$\begin{array}{c}(1,1) \\
0,95\end{array}$}} \\
\hline p-valor & & & & & & & & & & & & & & & & \\
\hline$(\mathbf{j}, \mathbf{k})$ & \multirow{2}{*}{\multicolumn{8}{|c|}{$\begin{array}{c}(0,0) \\
0,81\end{array}$}} & \multirow{2}{*}{\multicolumn{8}{|c|}{$\begin{array}{l}(0,0) \\
\mathbf{0 , 0 1}\end{array}$}} \\
\hline p-valor & & & & & & & & & & & & & & & & \\
\hline Coef. Escala & \multirow{2}{*}{\multicolumn{8}{|c|}{$\begin{array}{c}(0,0) \\
0,22\end{array}$}} & \multirow{2}{*}{\multicolumn{8}{|c|}{$\begin{array}{c}(0,0) \\
0,22\end{array}$}} \\
\hline p-valor & & & & & & & & & & & & & & & & \\
\hline
\end{tabular}
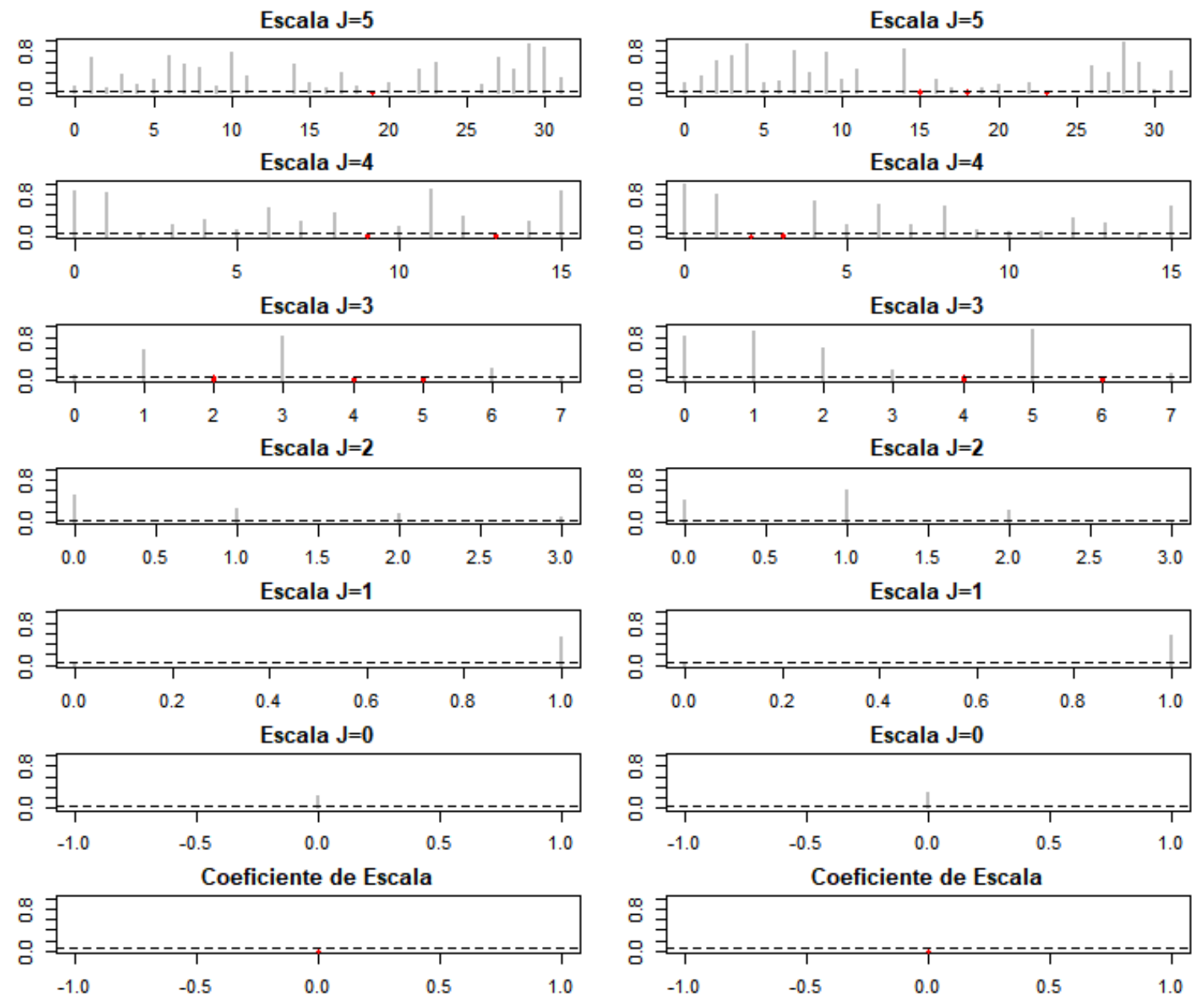

Figura 6.15: TDO: p-valores dos testes de sinal em comum nos expostos e asmáticos para cada indice $(j, k)$. Extremal Phase (D8) a esquerda e Least Asymmetric (LA8) a direita. 
Fixando os indivíduos não asmáticos e expostos ao fumo domiciliar os resultados dos testes se encontram na Figura 6.16
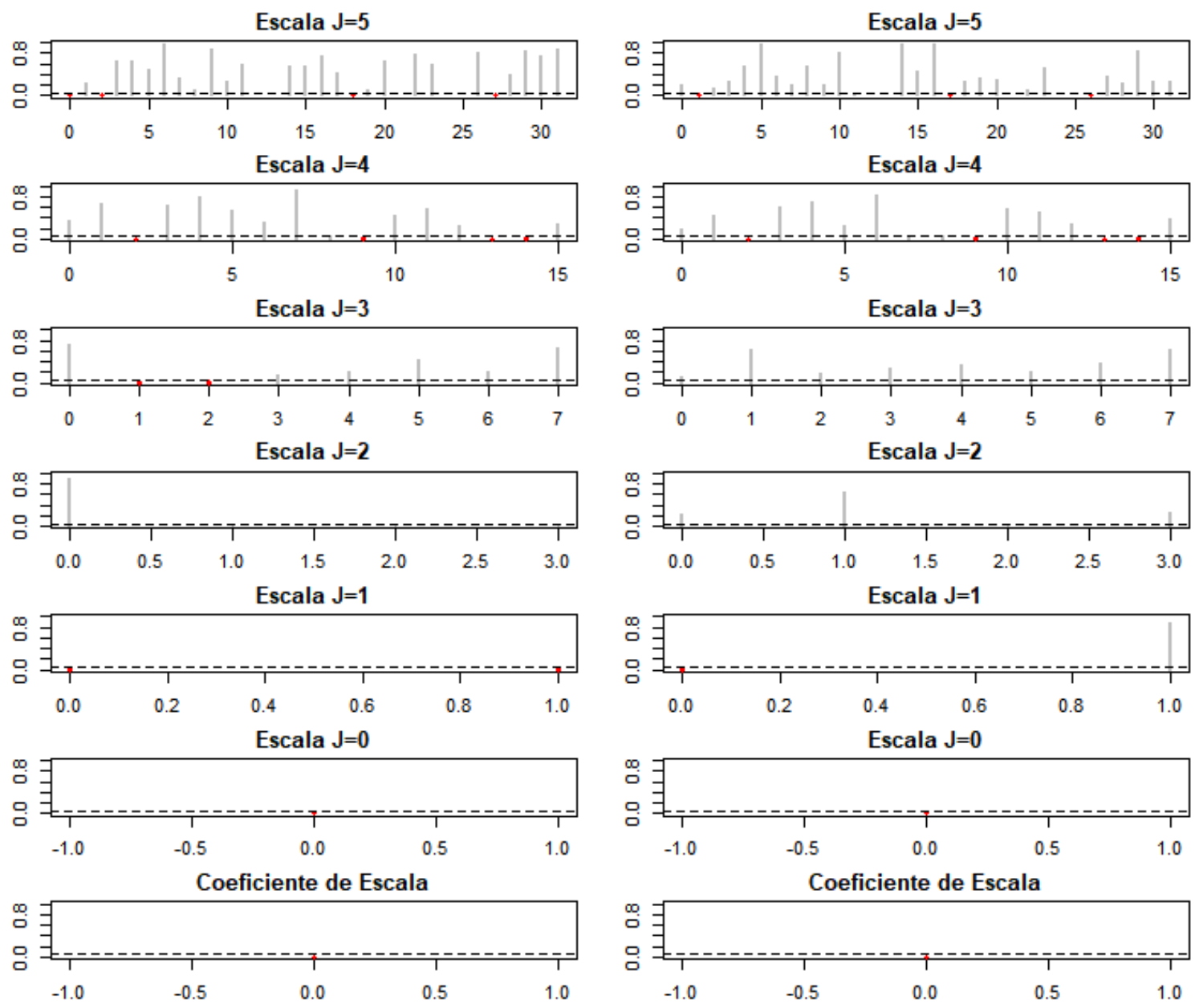

Figura 6.16: TDO: p-valores dos testes de sinal em comum nos expostos e não asmáticos para cada indice $(j, k)$. Extremal Phase (D8) a esquerda e Least Asymmetric (LA8) a direita.

Fixando os indivíduos asmáticos e não expostos ao fumo domiciliar, os resultados dos testes se encontram na Figura 6.17.

Fixando os indivíduos não asmáticos e não expostos ao fumo domiciliar, os resultados dos testes se encontram na Figura 6.18

Em todos os cenários foram encontrados efeitos de sinal em comum entre os indivíduos. Os sinais em comum foram obtidos na maioria dos níveis, o mesmo fato ocorreu para os indivíduos não expostos em ambas ondaletas.

\subsubsection{ANOVA utilizando a transformada discreta de ondaletas não decimada}

Como a transformada discreta de ondaletas não decimada possui forte autocorrelação, antes da aplicação da ANOVA foi utilizado a transformação 4.40 e depois aplicado a função logarítmica para diminuir esse efeito. Mesmo após a transformação 4.40, a autocorrelação em escalas mais grossas é alta, por isso foi utilizado $J_{0}=3$. Além disso, os coeficientes de escala também não foram utilizados na análise devido à autocorrelação significativa. Nesse 

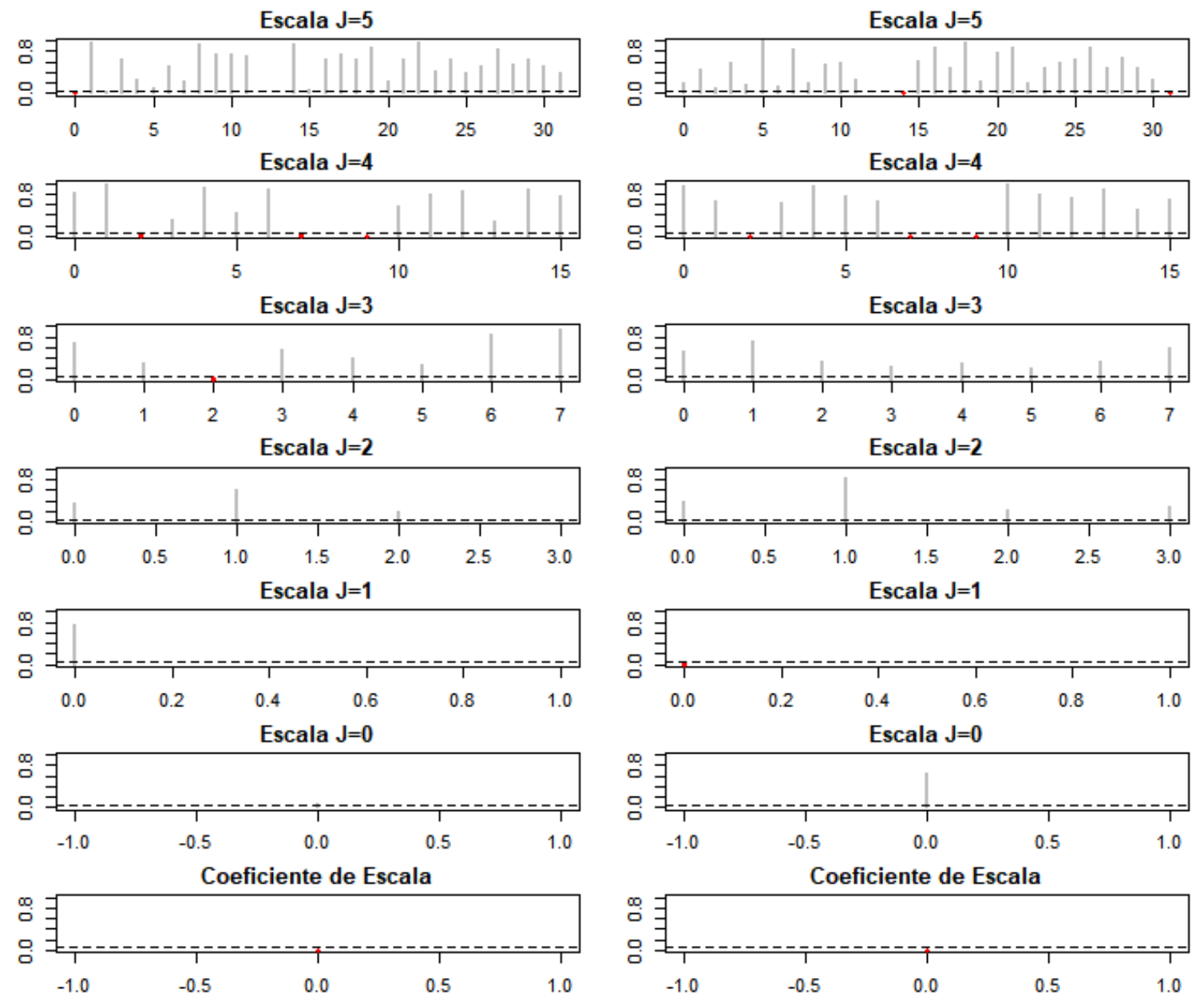

Figura 6.17: TDO: p-valores dos testes de sinal em comum nos não expostos e asmáticos para cada indice $(j, k)$. Extremal Phase (D8) a esquerda e Least Asymmetric (LA8) a direita.

caso, o modelo pode ser reescrito da seguinte maneira:

$\left.\log _{2} W_{\ell m s}(j, k)=\theta(j, k)\right)+\tau(j, k) \times I\left(\right.$ Asma $\left._{\ell m s}\right)+\eta(j, k) \times I\left(F u m o_{\ell m s}\right)+\delta(j, k) \times I\left(\right.$ Asma $\left._{\ell m s}\right) I\left(F u m o_{\ell m s}\right)+\varepsilon_{\ell m s}(j, k)$,

em que $\log _{2} W_{\ell m s}(j, k)$ é o logaritmo da transformação 4.40 da TDOND do Pico de Fluxo Expiratório da $\ell$-ésima unidade amostral dentro dos níveis $m$ e $s$ nos índices $(j, k), \theta(j, k)$ é o sinal em comum nos índices $(j, k), \tau$ é o efeito da asma nos índices $(j, k), \eta(j, k)$ é o efeito do fumo domiciliar nos índices $(j, k), \delta(j, k)$ é o efeito da interação entre asma e fumo domiciliar nos índices $(j, k), \varepsilon_{\ell m s}(j, k)$ é um termo de erro aleatório.

Primeiramente, iremos testar se o efeito de interação entre o fumo e a asma são significativos ou não, ou seja

$$
\left\{\begin{array}{l}
H_{0}: \delta(j, k)=0 \\
H_{1}: \delta(j, k) \neq 0
\end{array}\right.
$$

Os resultados para os índices $(j, k)$ são apresentados na Figura 6.19 e na Tabela 6.7.

Pelas 6.19 e Tabela 6.7, podemos observar que, em ambas ondaletas foram obtidos efeitos de interação significativos tanto no nível $J=4$ e $J=5$. Esses resultados convergem com o que foi obtido nas TDO e TDF. 

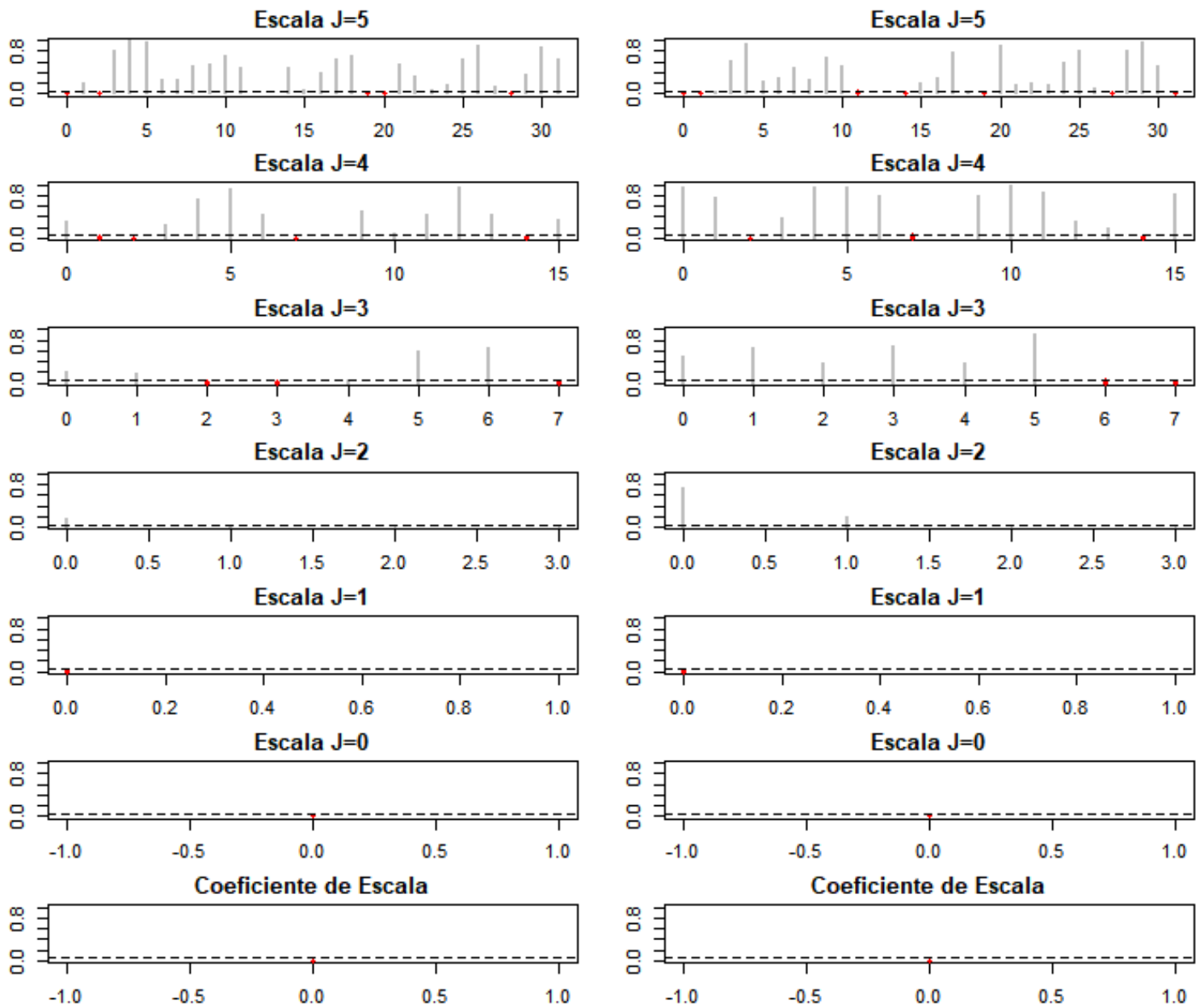

Figura 6.18: TDO: p-valores dos testes de sinal em comum nos não expostos e não asmáticos para cada indice $(j, k)$. Extremal Phase (D8) a esquerda e Least Asymmetric (LA8) a direita.

Como anteriormente, vamos estratificar a amostra em dois subgrupos: expostos ao fumo e não expostos ao fumo e então aplicar a ANOVA para verificar o efeito da asma. Para isso, vamos testar,

$$
\left\{\begin{array}{l}
H_{0}: \tau(j, k)=0 \\
H_{1}: \tau(j, k) \neq 0
\end{array}\right.
$$

Primeiramente, fixando os indivíduos expostos ao fumo domiliciar, os resultados dos testes se encontram na Figura 6.20 e na Tabela 6.8.

Agora, fixando os indivíduos expostos não ao fumo domiciliar, os resultados dos testes se encontram na Figura 6.21 e na Tabela 6.9.

Foram encontradas diferenças significativas do efeito da asma nos dois subgrupos. Entre os expostos ao fumo foram encontradas diferenças significativas no nível $J=4$ em ambas as ondaletas. Entre os não expostos ao fumo foram encontradas diferenças significativas nos níveis $J=4$ e $J=3$ em ambas ondaletas. Nos níveis de resolução que podemos comparar, os resultado convergem com os obtidos na TDO e na TDF.

Por último iremos testar efeito de sinal em comum entre as séries, foram criados quatro subgrupos, os asmáticos e expostos, não asmáticos e expostos, asmáticos e não expostos e os não asmáticos e não expostos. Para isso, vamos testar a seguinte hipótese, 

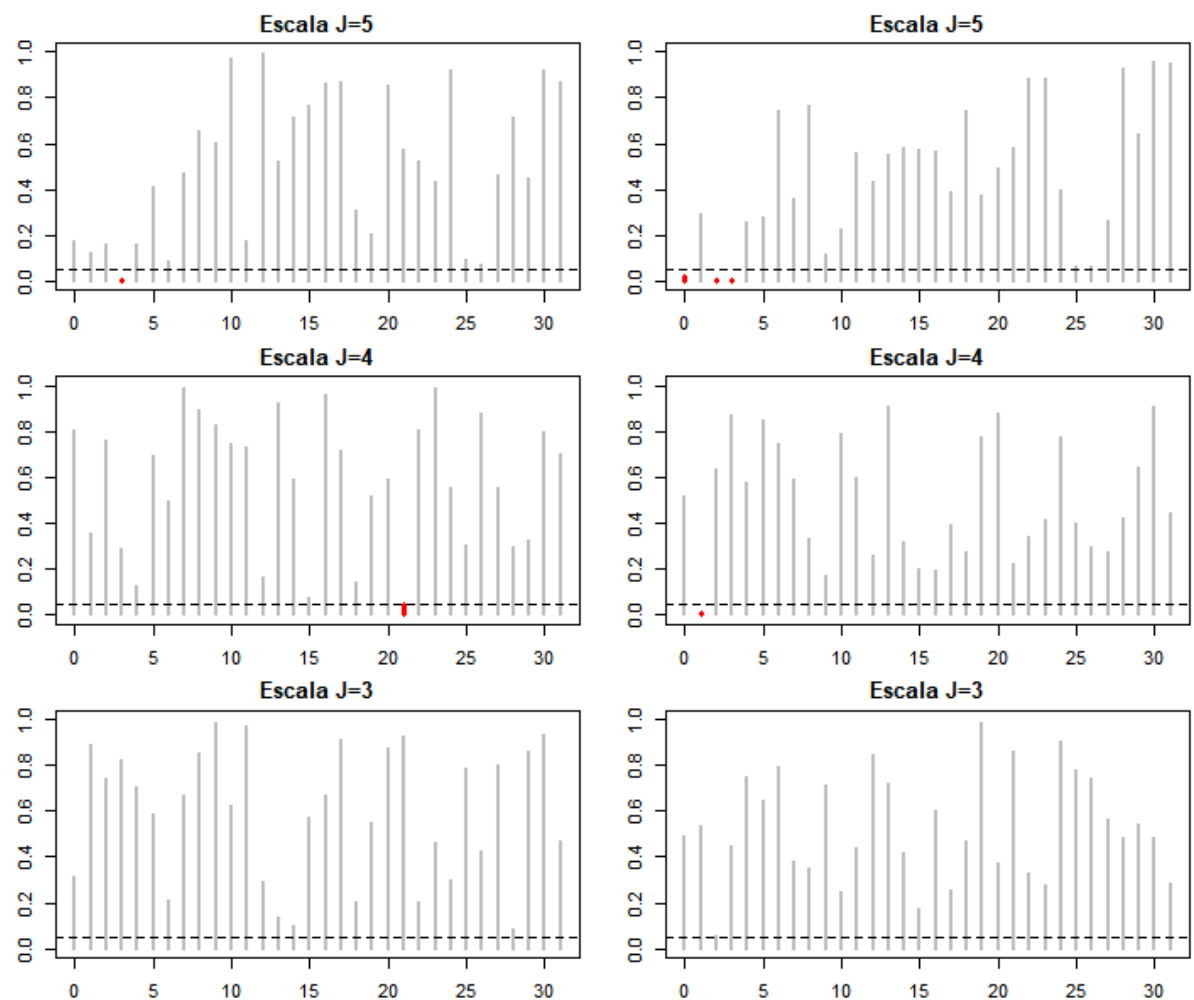

Figura 6.19: TDOND: p-valores dos testes de interação para cada índice $(j, k)$. Extremal Phase (D8) a esquerda e Least Asymmetric (LA8) a direita.

Tabela 6.7: TDOND: p-valores dos testes de interação para cada indice $(j, k)$.

\begin{tabular}{|c|c|c|c|c|c|c|c|c|c|c|c|c|c|c|c|c|}
\hline & \multicolumn{8}{|c|}{ Extremal Phase (D8) } & \multicolumn{8}{|c|}{ Least Asymmetric (LA8) } \\
\hline$(\mathbf{j}, \mathbf{k})$ & $(5,0)$ & $(5,1)$ & $(5,2)$ & $(5,3)$ & $(5,4)$ & $(5,5)$ & $(5,6)$ & $(5,7)$ & $(5,0)$ & $(5,1)$ & $(5,2)$ & $(5,3)$ & $(5,4)$ & $(5,5)$ & $(5,6)$ & $(5,7)$ \\
\hline p-valor & 0,18 & 0,13 & 0,16 & 0,01 & 0,16 & 0,41 & 0,09 & 0,47 & 0,02 & 0,29 & 0,01 & 0,01 & 0,26 & 0,28 & 0,75 & 0,36 \\
\hline$(\mathbf{j}, \mathbf{k})$ & $(5,8)$ & $(5,9)$ & $(5,10)$ & $(5,11)$ & $(5,12)$ & $(5,13)$ & $5,14)$ & $(5,15)$ & $(5,8)$ & $(5,9)$ & $(5,10)$ & $(5,11)$ & $(5,12)$ & $(5,13)$ & $(5,14)$ & $(5,15)$ \\
\hline p-valor & 0,66 & 0,60 & 0,98 & 0,18 & 0,99 & 0,52 & 0,71 & 0,77 & 0,77 & 0,12 & 0,23 & 0,56 & 0,44 & 0,55 & 0,58 & 0,57 \\
\hline$(\mathbf{j}, \mathbf{k})$ & $(5,16)$ & $(5,17)$ & $(5,18)$ & $(5,19)$ & $(5,20)$ & $(5,21)$ & $(5,22)$ & $(5,23)$ & $(5,16)$ & $(5,17)$ & $(5,18)$ & $(5,19)$ & $(5,20)$ & $(5,21)$ & $(5,22)$ & $(5,23)$ \\
\hline p-valor & 0,86 & 0,87 & 0,31 & 0,20 & 0,86 & 0,58 & 0,52 & 0,43 & 0,56 & 0,39 & 0,74 & 0,38 & 0,49 & 0,58 & 0,88 & 0,88 \\
\hline$(\mathbf{j}, \mathbf{k})$ & $(5,24)$ & $(5,25)$ & $(5,26)$ & $(5,27)$ & $(5,28)$ & $(5,29)$ & $(5,30)$ & $(5,31)$ & $(5,24)$ & $(5,25)$ & $(5,26)$ & $(5,27)$ & $(5,28)$ & $(5,29)$ & $(5,30)$ & $(5,31)$ \\
\hline p-valor & 0,92 & 0,09 & 0,07 & 0,46 & 0,71 & 0,45 & 0,92 & 0,87 & 0,40 & 0,07 & 0,07 & 0,26 & 0,93 & 0,64 & 0,96 & 0,95 \\
\hline$(\mathbf{j}, \mathbf{k})$ & $(4,0)$ & $(4,1)$ & $(4,2)$ & $(4,3)$ & $(4,4)$ & $(4,5)$ & $(4,6)$ & $(4,7)$ & $(4,0)$ & $(4,1)$ & $(4,2)$ & $(4,3)$ & $(4,4)$ & $(4,5)$ & $(4,6)$ & $(4,7)$ \\
\hline p-valor & 0,81 & 0,35 & 0,76 & 0,29 & 0,13 & 0,69 & 0,50 & 0,99 & 0,52 & 0,01 & 0,64 & 0,88 & 0,57 & 0,85 & 0,75 & 0,60 \\
\hline$(\mathbf{j}, \mathbf{k})$ & $(4,8)$ & $(4,9)$ & $(4,10)$ & $(4,11)$ & $(4,12)$ & $(4,13)$ & $(4,14)$ & $(4,15)$ & $(4,8)$ & $(4,9)$ & $(4,10)$ & $(4,11)$ & $(4,12)$ & $(4,13)$ & $(4,14)$ & $(4,15)$ \\
\hline p-valor & 0,89 & 0,83 & 0,74 & 0,73 & 0,17 & 0,92 & 0,59 & 0,08 & 0,33 & 0,17 & 0,79 & 0,60 & 0,26 & 0,91 & 0,32 & 0,20 \\
\hline$(\mathbf{j}, \mathbf{k})$ & 4,16 & & $(4,18)$ & & & & $(4$, & & $(4,16)$ & & $(4,1$ & & & & & $(4,23)$ \\
\hline p-valor & 0,96 & 0,72 & 0,15 & 0,52 & 0,59 & 0,05 & 0,80 & 0,99 & 0,20 & 0,39 & 0,28 & 0,77 & 0,88 & 0,22 & 0,34 & 0,42 \\
\hline$(\mathbf{j}, \mathbf{k})$ & $(4,24)$ & $(4,25)$ & $(4,26)$ & & & & $(4,30)$ & $(4,31)$ & $(4,24)$ & & $(4,26)$ & & & & & $(4,31)$ \\
\hline p-valor & 0,55 & 0,31 & 0,88 & 0,56 & 0,30 & 0,32 & 0,80 & 0,70 & 0,78 & 0,40 & 0,30 & 0,27 & 0,42 & 0,64 & 0,91 & 0,45 \\
\hline$(\mathbf{j}, \mathbf{k})$ & $(3,0)$ & $(3,1)$ & $(3,2)$ & $(3,3)$ & $(3,4)$ & & $(36)$ & $(3,7)$ & $(3,0)$ & & $(3,2)$ & & & & $(3,6)$ & $(3,7)$ \\
\hline p-valor & 0,31 & 0,89 & 0,74 & 0,82 & 0,70 & 0,58 & 0,21 & 0,67 & 0,49 & 0,54 & 0,06 & 0,45 & 0,75 & 0,65 & 0,79 & 0,38 \\
\hline$(\mathrm{j}, \mathrm{k}$ & $3,8)$ & $(3$, & ( & & & & & & $(3,8)$ & & $(3,10)$ & & & & 14) & $(3,15)$ \\
\hline p-valor & 0,85 & 0,99 & 0,62 & 0,97 & 0,29 & 0,14 & 0,10 & 0,57 & 0,35 & 0,71 & 0,25 & 0,44 & 0,84 & 0,72 & 0,42 & 0,17 \\
\hline$(\mathbf{j}, \mathbf{k})$ & $3,16)$ & & $(3,18)$ & & & & & & & & $(3,18)$ & & & & & $(3,23)$ \\
\hline p-valor & 0,67 & 0,91 & 0,20 & 0,55 & 0,87 & 0,92 & 0,20 & 0,46 & 0,61 & 0,26 & 0,47 & 0,99 & 0,37 & 0,86 & 0,33 & 0,27 \\
\hline$(\mathbf{j}, \mathbf{k})$ & $3,24)$ & $(3,25)$ & $(3,26)$ & & & & & & & & $(3,26)$ & & & & & \\
\hline p-valor & 0,30 & 0,79 & 0,42 & 0,80 & 0,09 & 0,86 & 0,93 & 0,47 & 0,90 & 0,78 & 0,74 & 0,57 & 0,48 & 0,55 & 0,48 & 0,28 \\
\hline
\end{tabular}

$$
\left\{\begin{array}{l}
H_{0}: \theta(j, k)=0, \\
H_{1}: \theta(j, k) \neq 0 .
\end{array} .\right.
$$

Fixando os indivíduos asmáticos e expostos ao fumo domiciliar, os resultados dos testes se encontram na Figura 6.22. 

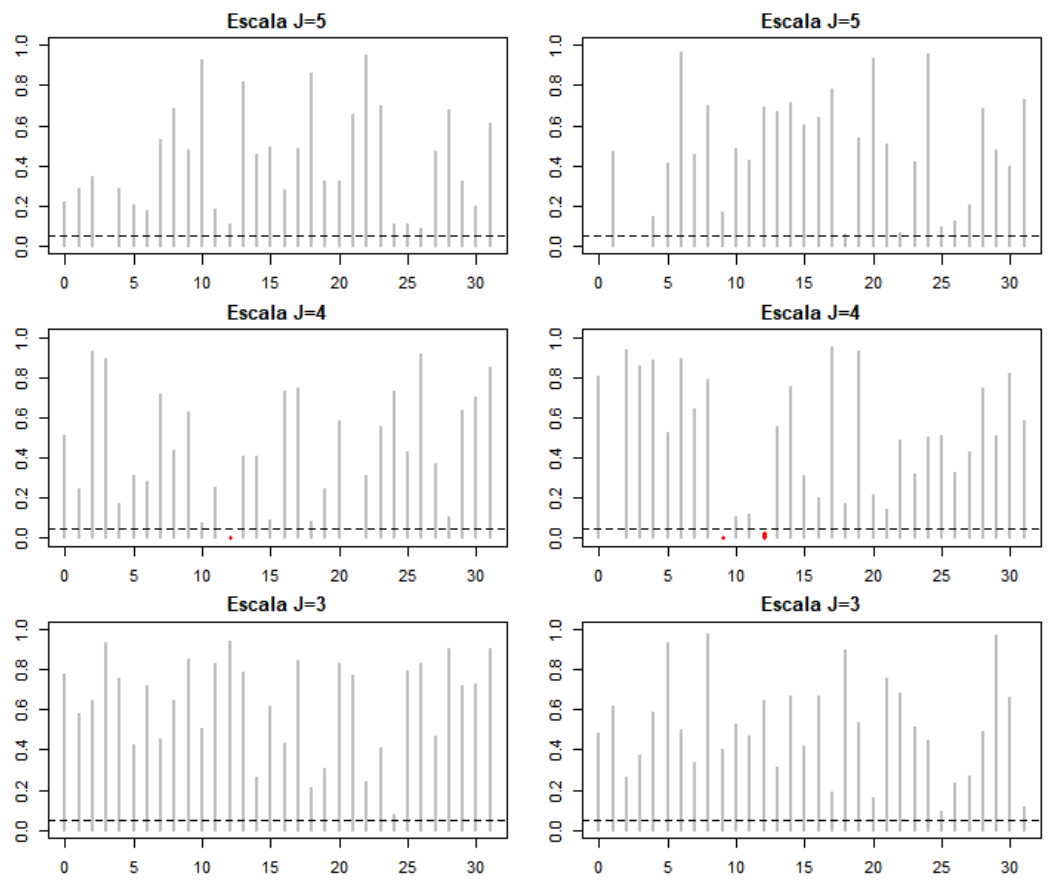

Figura 6.20: TDOND: p-valores dos testes do efeito da asma nos expostos para cada indice $(j, k)$. Extremal Phase (D8) a esquerda e Least Asymmetric (LA8) a direita.

Tabela 6.8: TDOND: p-valores dos testes do efeito da asma nos expostos para cada indice $(j, k)$.

\begin{tabular}{|c|c|c|c|c|c|c|c|c|c|c|c|c|c|c|c|c|}
\hline & \multicolumn{8}{|c|}{ Extremal Phase (D8) } & \multicolumn{8}{|c|}{ Least Asymmetric (LA8) } \\
\hline$(\mathbf{j}, \mathbf{k})$ & $(5,0)$ & $(5,1)$ & $(5,2)$ & $(5,3)$ & $(5,4)$ & $(5,5)$ & $(5,6)$ & $(5,7)$ & $(5,0)$ & $(5,1)$ & $(5,2)$ & $(5,3)$ & $(5,4)$ & $(5,5)$ & $(5,6)$ & $(5,7)$ \\
\hline p-valor & 0,23 & 0,29 & 0,35 & - & 0,28 & 0,21 & 0,17 & 0,53 & & 0,52 & - & - & 0,90 & 0,50 & 0,54 & 0,73 \\
\hline$(\mathbf{j}, \mathbf{k})$ & $(5,8)$ & $(5,9)$ & $(5,10)$ & $(5,11)$ & $(5,12)$ & $(5,13)$ & $(5,14)$ & $(5,15)$ & $(5,8)$ & $(5,9)$ & $(5,10)$ & $(5,11)$ & $(5,12)$ & $(5,13)$ & $(5,14)$ & $(5,15)$ \\
\hline p-valor & 0,69 & 0,48 & 0,93 & 0,19 & 0,11 & 0,82 & 0,45 & 0,50 & 0,70 & 0,17 & 0,49 & 0,43 & 0,69 & 0,67 & 0,71 & 0,61 \\
\hline$(\mathbf{j}, \mathbf{k})$ & $(5,16)$ & $(5,17)$ & $(5,18)$ & $(5,19)$ & $(5,20)$ & $(5,21)$ & $(5,22)$ & $(5,23)$ & $(5,16)$ & $(5,17)$ & $(5,18)$ & $(5,19)$ & $(5,20)$ & $(5,21)$ & $(5,22)$ & $(5,23)$ \\
\hline p-valor & 0,28 & 0,49 & 0,86 & 0,32 & 0,32 & 0,65 & 0,95 & 0,70 & 0,64 & 0,78 & 0,06 & 0,53 & 0,93 & 0,51 & 0,07 & 0,42 \\
\hline$(\mathbf{j}, \mathbf{k})$ & $(5,24)$ & $(5,25)$ & $(5,26)$ & $(5,27)$ & $(5,28)$ & $(5,29)$ & $(5,30)$ & $(5,31)$ & $(5,24)$ & $(5,25)$ & $(5,26)$ & $(5,27)$ & $(5,28)$ & $(5,29)$ & $(5,30)$ & $(5,31)$ \\
\hline p-valor & 0,11 & 0,11 & 0,09 & 0,47 & 0,68 & 0,32 & 0,20 & 0,61 & 0,96 & 0,09 & 0,13 & 0,21 & 0,68 & 0,48 & 0,40 & 0,73 \\
\hline$(\mathbf{j}, \mathbf{k})$ & $(4,0)$ & $(4,1)$ & $(4,2)$ & $(4,3)$ & $(4,4)$ & $(4,5)$ & $(4,6)$ & $(4,7)$ & $(4,0)$ & $(4,1)$ & $(4,2)$ & $(4,3)$ & $(4,4)$ & $(4,5)$ & $(4,6)$ & $(4,7)$ \\
\hline p-valor & 0,51 & 0,25 & 0,93 & 0,89 & 0,17 & 0,31 & 0,28 & 0,71 & 0,81 & - & 0,94 & 0,86 & 0,88 & 0,52 & 0,89 & 0,64 \\
\hline$(\mathbf{j}, \mathbf{k})$ & $(4,8)$ & $(4,9)$ & $(4,10)$ & $(4,11)$ & $(4,12)$ & $(4,13)$ & $(4,14)$ & $(4,15)$ & $(4,8)$ & $(4,9)$ & $(4,10)$ & $(4,11)$ & $(4,12)$ & $(4,13)$ & $(4,14)$ & $(4,15)$ \\
\hline p-valor & 0,44 & 0,63 & 0,08 & 0,25 & 0,01 & 0,41 & 0,40 & 0,09 & 0,79 & 0,01 & 0,11 & 0,12 & 0,02 & 0,56 & 0,76 & 0,31 \\
\hline$(\mathbf{j}, \mathbf{k})$ & $(4,16)$ & $(4,17)$ & $(4,18)$ & $(4,19)$ & $(4,20)$ & $(4,21)$ & $(4,22)$ & $(4,23)$ & $(4,16)$ & $(4,17)$ & $(4,18)$ & $(4,19)$ & $(4,20)$ & $(4,21)$ & $(4,22)$ & $(4,23)$ \\
\hline p-valor & 0,73 & 0,75 & 0,08 & 0,25 & 0,58 & - & 0,32 & 0,55 & 0,20 & 0,95 & 0,17 & 0,93 & 0,21 & 0,15 & 0,49 & 0,32 \\
\hline$(\mathbf{j}, \mathbf{k})$ & $(4,24)$ & $(4,25)$ & $(4,26)$ & $(4,27)$ & $(4,28)$ & $(4,29)$ & $(4,30)$ & $(4,31)$ & $(4,24)$ & $(4,25)$ & $(4,26)$ & $(4,27)$ & $(4,28)$ & $(4,29)$ & $(4,30)$ & $(4,31)$ \\
\hline p-valor & 0,73 & 0,43 & 0,91 & 0,37 & 0,11 & 0,63 & 0,70 & 0,85 & 0,51 & 0,51 & 0,32 & 0,43 & 0,75 & 0,51 & 0,82 & 0,58 \\
\hline$(\mathbf{j}, \mathbf{k})$ & $(3,0)$ & $(3,1)$ & $(3,2)$ & $(3,3)$ & $(3,4)$ & $(3,5)$ & $(3,6)$ & $(3,7)$ & $(3,0)$ & $(3,1)$ & $(3,2)$ & $(3,3)$ & $(3,4)$ & $(3,5)$ & $(3,6)$ & $(3,7)$ \\
\hline p-valor & 0,78 & 0,58 & 0,65 & 0,94 & 0,75 & 0,43 & 0,72 & 0,45 & 0,48 & 0,62 & 0,26 & 0,37 & 0,59 & 0,94 & 0,50 & 0,34 \\
\hline$(\mathbf{j}, \mathbf{k})$ & $(3,8)$ & $(3,9)$ & $(3,10)$ & $(3,11)$ & $(3,12)$ & $(3,13)$ & $(3,14)$ & $(3,15)$ & $(3,8)$ & $(3,9)$ & $(3,10)$ & $(3,11)$ & $(3,12)$ & $(3,13)$ & $(3,14)$ & $(3,15)$ \\
\hline p-valor & 0,64 & 0,85 & 0,51 & 0,83 & 0,94 & 0,78 & 0,26 & 0,62 & 0,98 & 0,40 & 0,53 & 0,47 & 0,65 & 0,32 & 0,67 & 0,42 \\
\hline$(\mathbf{j}, \mathbf{k})$ & $(3,16)$ & $(3,17)$ & $(3,18)$ & $(3,19)$ & $(3,20)$ & $(3,21)$ & $(3,22)$ & $(3,23)$ & $(3,16)$ & $(3,17)$ & $(3,18)$ & $(3,19)$ & $(3,20)$ & $(3,21)$ & $(3,22)$ & $(3,23)$ \\
\hline p-valor & 0,43 & 0,85 & 0,21 & 0,30 & 0,83 & 0,77 & 0,24 & 0,41 & 0,67 & 0,19 & 0,89 & 0,54 & 0,16 & 0,75 & 0,68 & 0,52 \\
\hline$(\mathbf{j}, \mathbf{k})$ & $(3,24)$ & $(3,25)$ & $(3,26)$ & $(3,27)$ & $(3,28)$ & $(3,29)$ & $(3,30)$ & $(3,31)$ & $(3,24)$ & $(3,25)$ & $(3,26)$ & $(3,27)$ & & $(3,29)$ & & $(3,31)$ \\
\hline p-valor & 0,08 & 0,80 & 0,83 & 0,47 & 0,90 & 0,72 & 0,73 & 0,90 & 0,45 & 0,10 & 0,23 & 0,27 & 0,49 & 0,97 & 0,66 & 0,12 \\
\hline
\end{tabular}

Fixando os indivíduos não asmáticos e expostos ao fumo domiciliar, os resultados dos testes se encontram na Figura 6.23.

Fixando os indivíduos asmáticos e não expostos ao fumo domiciliar, os resultados dos testes se encontram na Figura 6.24.

Fixando os indivíduos não asmáticos e não expostos ao fumo domiciliar, os resultados dos testes se encontram na Figura 6.25.

Foram encontrados sinais em comum em todos os subgrupos analisados, em ambas as 

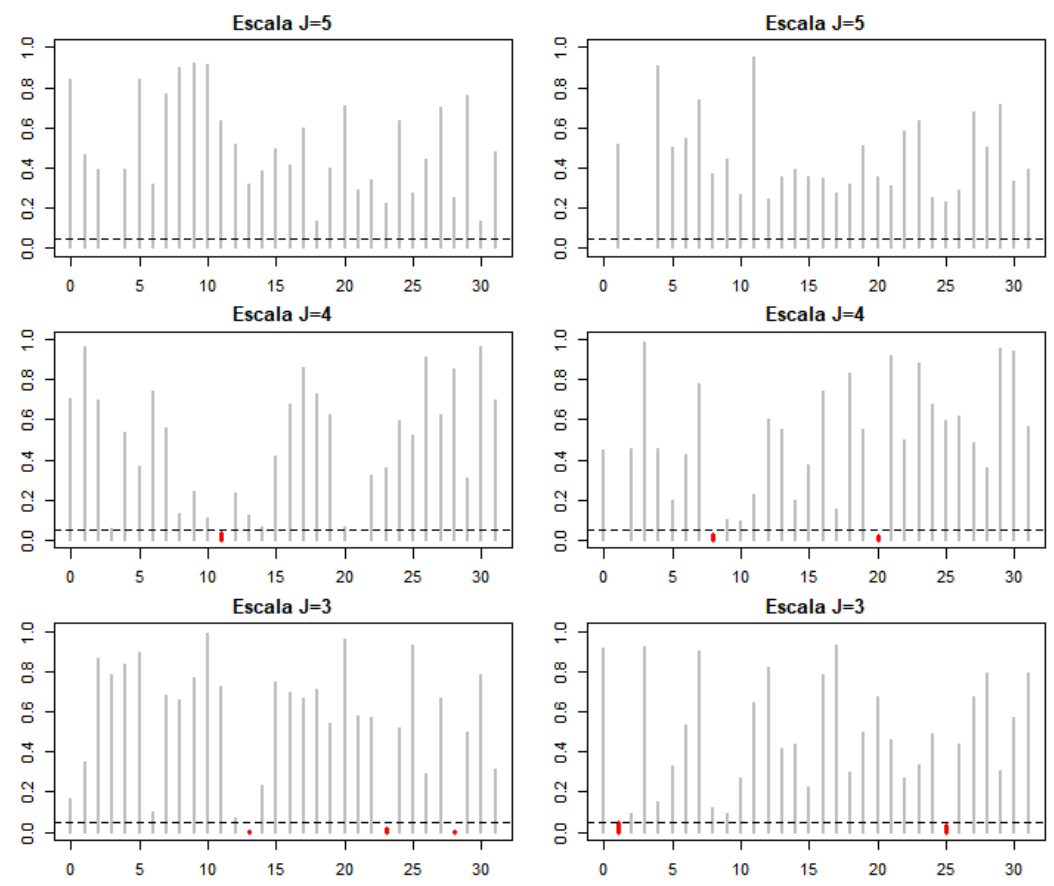

Figura 6.21: TDOND: p-valores dos testes do efeito da asma nos não expostos para cada indice $(j, k)$. Extremal Phase (D8) a esquerda e Least Asymmetric (LA8) a direita.

Tabela 6.9: TDOND: p-valores dos testes do efeito da asma nos não expostos para cada índice $(j, k)$.

\begin{tabular}{|c|c|c|c|c|c|c|c|c|c|c|c|c|c|c|c|c|}
\hline & \multicolumn{8}{|c|}{ Extremal Phase (D8) } & \multicolumn{8}{|c|}{ Least Asymmetric (LA8) } \\
\hline$(\mathrm{j}, \mathrm{k})$ & $(5,0)$ & $(5,1)$ & $(5,2)$ & $(5,3)$ & $(5,4)$ & $(5,5)$ & $(5,6)$ & $(5,7)$ & $(5,0)$ & $(5,1)$ & $(5,2)$ & $(5,3)$ & $(5,4)$ & $(5,5)$ & $(5,6)$ & $(5,7)$ \\
\hline p-valor & 0,84 & 0,47 & 0,39 & - & 0,39 & 0,84 & 0,32 & 0,77 & - & 0,52 & & - & 0,90 & 0,50 & 0,54 & 0,73 \\
\hline$(\mathbf{j}, \mathbf{k})$ & $(5,8)$ & $(5,9)$ & $(5,10)$ & $(5,11)$ & $(5,12)$ & $(5,13)$ & $(5,14)$ & $(5,15)$ & $(5,8)$ & $(5,9)$ & $(5,10)$ & $(5,11)$ & $(5,12)$ & $(5,13)$ & $(5,14)$ & $(5,15)$ \\
\hline p-valor & 0,90 & 0,92 & 0,91 & 0,63 & 0,52 & 0,32 & 0,39 & 0,49 & 0,37 & 0,45 & 0,27 & 0,95 & 0,25 & 0,36 & 0,39 & 0,36 \\
\hline$(\mathbf{j}, \mathbf{k})$ & $(5,16)$ & $(5,17)$ & $(5,18)$ & $(5,19)$ & $(5,20)$ & $(5,21)$ & $(5,22)$ & $(5,23)$ & $(5,16)$ & $(5,17)$ & $(5,18)$ & $(5,19)$ & $(5,20)$ & $(5,21)$ & $(5,22)$ & $(5,23)$ \\
\hline p-valor & 0,41 & 0,60 & 0,14 & 0,40 & 0,71 & 0,29 & 0,34 & 0,22 & 0,35 & 0,28 & 0,32 & 0,51 & 0,36 & 0,31 & 0,58 & 0,64 \\
\hline$(\mathbf{j}, \mathbf{k})$ & $(5,24)$ & $(5,25)$ & $(5,26)$ & $(5,27)$ & $(5,28)$ & $(5,29)$ & $(5,30)$ & $(5,31)$ & $(5,24)$ & $(5,25)$ & $(5,26)$ & $(5,27)$ & $(5,28)$ & $(5,29)$ & $(5,30)$ & $(5,31)$ \\
\hline p-valor & 0,64 & 0,27 & 0,44 & 0,70 & 0,25 & 0,76 & 0,14 & 0,48 & 0,26 & 0,23 & 0,29 & 0,68 & 0,50 & 0,72 & 0,33 & 0,39 \\
\hline$(\mathbf{j}, \mathbf{k})$ & $(4,0)$ & $(4,1)$ & $(4,2)$ & $(4,3)$ & $(4,4)$ & $(4,5)$ & $(4,6)$ & $(4,7)$ & $(4,0)$ & $(4,1)$ & $(4,2)$ & $(4,3)$ & $(4,4)$ & $(4,5)$ & $(4,6)$ & $(4,7)$ \\
\hline p-valor & 0,71 & 0,97 & 0,70 & 0,06 & 0,54 & 0,37 & 0,74 & 0,56 & 0,45 & & 0,46 & 0,98 & 0,46 & 0,20 & 0,42 & 0,78 \\
\hline$(\mathbf{j}, \mathbf{k})$ & $(4,8)$ & $(4,9)$ & $(4,10)$ & $(4,11)$ & $(4,12)$ & $(4,13)$ & $(4,14)$ & $(4,15)$ & $(4,8)$ & $(4,9)$ & $(4,10)$ & $(4,11)$ & $(4,12)$ & $(4,13)$ & $(4,14)$ & $(4,15)$ \\
\hline p-valor & 0,13 & 0,24 & 0,11 & 0,04 & 0,23 & 0,12 & 0,07 & 0,42 & 0,03 & 0,10 & 0,09 & 0,23 & 0,60 & 0,55 & 0,20 & 0,37 \\
\hline$(\mathbf{j}, \mathbf{k})$ & $(4,16)$ & $(4,17)$ & $(4,18)$ & $(4,19)$ & $(4,20)$ & $(4,21)$ & $(4,22)$ & $(4,23)$ & $(4,16)$ & $(4,17)$ & $(4,18)$ & $(4,19)$ & $(4,20)$ & $(4,21)$ & $(4,22)$ & $(4,23)$ \\
\hline p-valor & 0,68 & 0,86 & 0,73 & 0,63 & 0,07 & - & 0,32 & 0,36 & 0,74 & 0,16 & 0,83 & 0,55 & 0,02 & 0,92 & 0,50 & 0,88 \\
\hline$(\mathbf{j}, \mathbf{k})$ & $(4,24)$ & $(4,25)$ & $(4,26)$ & $(4,27)$ & $(4,28)$ & $(4,29)$ & $(4,30)$ & $(4,31)$ & $(4,24)$ & $(4,25)$ & $(4,26)$ & $(4,27)$ & $(4,28)$ & $(4,29)$ & $(4,30)$ & $(4,31)$ \\
\hline p-valor & 0,59 & 0,52 & 0,91 & 0,62 & 0,85 & 0,31 & 0,96 & 0,70 & 0,68 & 0,59 & 0,62 & 0,48 & 0,36 & 0,95 & 0,94 & 0,56 \\
\hline$(\mathbf{j}, \mathbf{k})$ & $(3,0)$ & $(3,1)$ & $(3,2)$ & $(3,3)$ & $(3,4)$ & $(3,5)$ & $(3,6)$ & $(3,7)$ & $(3,0)$ & $(3,1)$ & $(3,2)$ & $(3,3)$ & $(3,4)$ & $(3,5)$ & $(3,6)$ & $(3,7)$ \\
\hline p-valor & 0,16 & 0,35 & 0,87 & 0,79 & 0,84 & 0,89 & 0,10 & 0,68 & 0,92 & 0,05 & 0,10 & 0,93 & 0,16 & 0,33 & 0,54 & 0,90 \\
\hline$(\mathbf{j}, \mathbf{k})$ & $(3,8)$ & $(3,9)$ & $(3,10)$ & $(3,11)$ & $(3,12)$ & $(3,13)$ & $(3,14)$ & $(3,15)$ & $(3,8)$ & $(3,9)$ & $(3,10)$ & $(3,11)$ & $(3,12)$ & $(3,13)$ & $(3,14)$ & $(3,15)$ \\
\hline p-valor & 0,66 & 0,77 & 0,99 & 0,72 & 0,07 & 0,01 & 0,23 & 0,75 & 0,13 & 0,09 & 0,27 & 0,64 & 0,82 & 0,41 & 0,44 & 0,23 \\
\hline$(\mathbf{j}, \mathbf{k})$ & $(3,16)$ & $(3,17)$ & $(3,18)$ & $(3,19)$ & $(3,20)$ & $(3,21)$ & $(3,22)$ & $(3,23)$ & $(3,16)$ & $(3,17)$ & $(3,18)$ & $(3,19)$ & $(3,20)$ & $(3,21)$ & $(3,22)$ & $(3,23)$ \\
\hline p-valor & 0,70 & 0,67 & 0,71 & 0,54 & 0,96 & 0,58 & 0,57 & 0,02 & 0,78 & 0,93 & 0,30 & 0,50 & 0,68 & 0,46 & 0,27 & 0,34 \\
\hline$(\mathbf{j}, \mathbf{k})$ & $(3,24)$ & $(3,25)$ & $(3,26)$ & $(3,27)$ & $(3,28)$ & $(3,29)$ & $(3,30)$ & $(3,31)$ & $(3,24)$ & $(3,25)$ & $(3,26)$ & $(3,27)$ & $(3,28)$ & $(3,29)$ & $(3,30)$ & $(3,31)$ \\
\hline p-valor & 0,52 & 0,93 & 0,29 & 0,66 & 0,01 & 0,49 & 0,79 & 0,31 & 0,49 & 0,03 & 0,44 & 0,68 & 0,79 & 0,31 & 0,57 & 0,79 \\
\hline
\end{tabular}

ondaletas e em todas as escalas. 

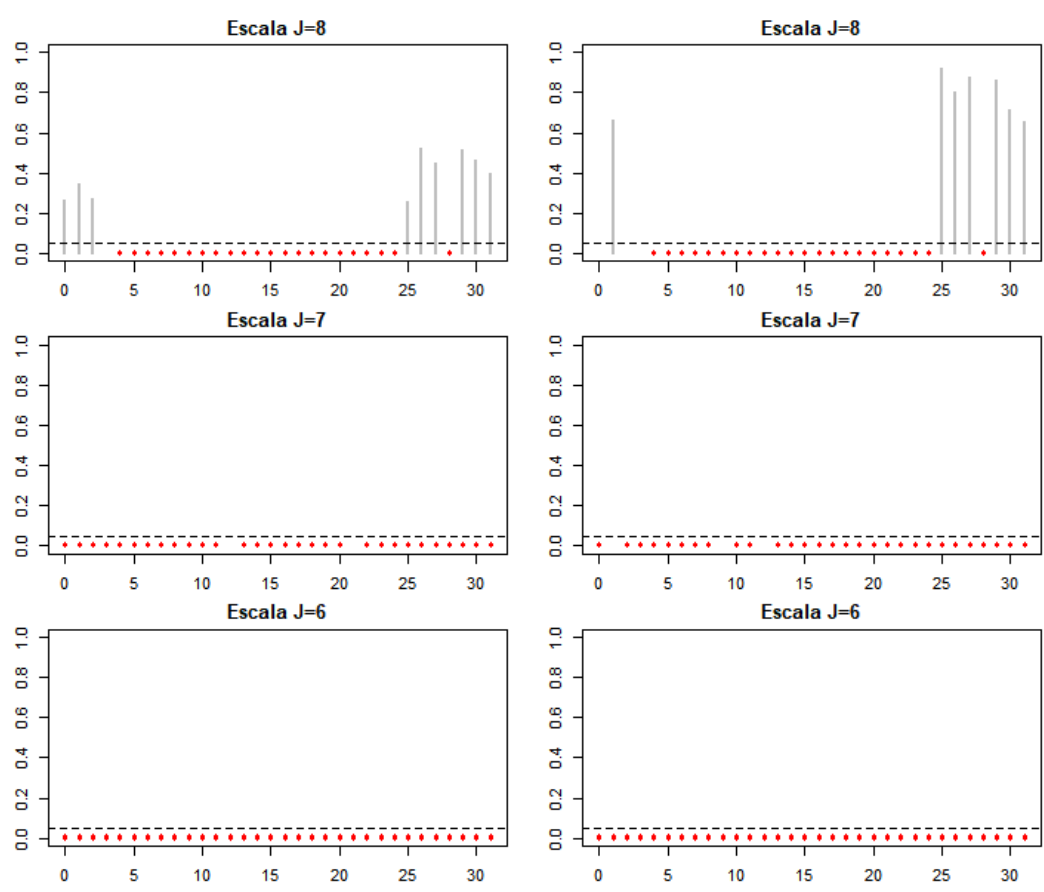

Figura 6.22: TDOND: p-valores dos testes de sinal em comum nos expostos e asmáticos para cada indice $(j, k)$. Extremal Phase (D8) a esquerda e Least Asymmetric (LA8) a direita.
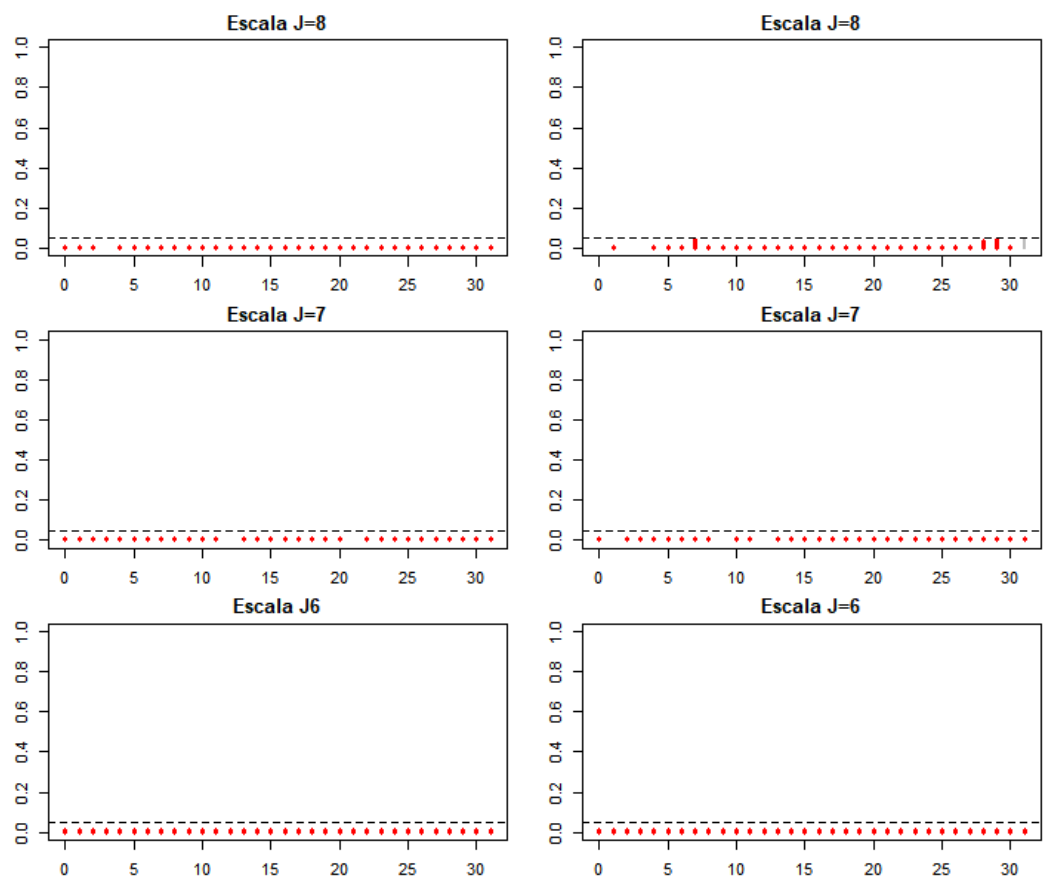

Figura 6.23: TDOND: p-valores dos testes de sinal em comum nos expostos e não asmáticos para cada indice $(j, k)$. Extremal Phase (D8) a esquerda e Least Asymmetric (LA8) a direita. 

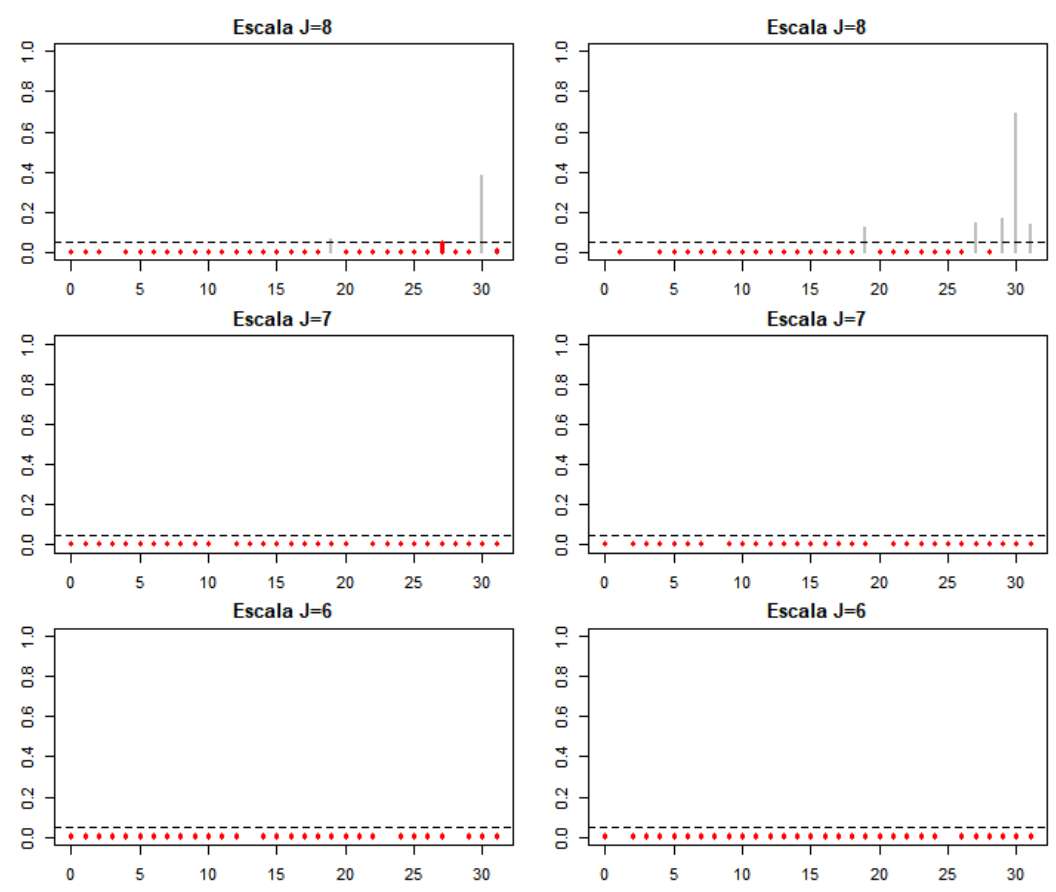

Figura 6.24: TDOND: p-valores dos testes de sinal em comum nos não expostos e asmáticos para cada indice $(j, k)$. Extremal Phase (D8) a esquerda e Least Asymmetric (LA8) a direita.
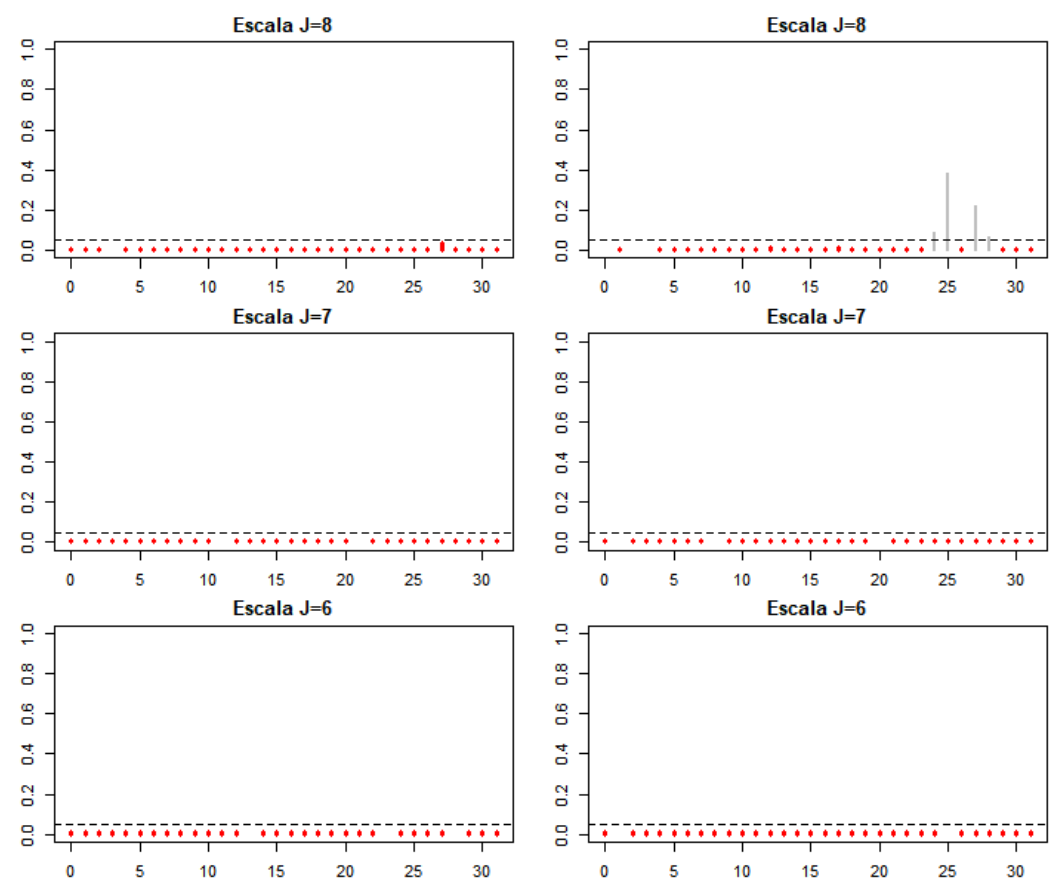

Figura 6.25: TDOND: p-valores dos testes de sinal em comum nos não expostos não asmáticos para cada indice $(j, k)$. Extremal Phase (D8) a esquerda e Least Asymmetric (LA8) a direita. 


\section{Capítulo 7}

\section{Conclusões}

Em ambas as metodologias estudadas obteve-se resultados aparentemente convergentes, apesar das simulações do poder do teste apontarem resultados um pouco diferentes. Vale salientar que os métodos das transformadas de ondaletas, tanto a transformada tradicional quanto a transformada não decimada, são teoricamente mais robustas em relação ao método da transformada de Fourier, pois a energia de uma série temporal não estacionária tende a se distribuir bem entre os coeficientes no caso de Fourier (devido seu domínio na frequência). Em contrapartida, as ondaletas tendem a concentrar melhor a energia da série em poucos coeficientes, o que teoricamente deveria evidenciar mais facilmente diferenças significativas nos testes de hipóteses. Um fator que pode ter influenciado foi a autocorrelação presente na transformada de ondaletas não decimada, pois apesar do uso da técnica para diminuir a auto dependência, esta não foi totalmente sanada. Outro ponto relevante é que com o uso da transformada de ondaletas não decimada, pode-se testar mais pontos se comparado as outras duas metodologias. Podendo assim, mais pontos com diferenças significativas serem detectados.

Como o resultado da aplicação aponta que não há muitas diferenças entre indivíduos com e sem asma quando estão expostos ao fumo domiciliar, podemos levantar a hipótese de que a exposição ao fumo é tão prejudicial para os não asmáticos quanto para os asmáticos, porém essa hipótese deveria ser avaliada com estudos mais bem controlados, sendo essa uma sugestão para trabalhos futuros. 
CONCLUSÕES 


\section{Apêndice A}

\section{Dados utilizados na aplicação}

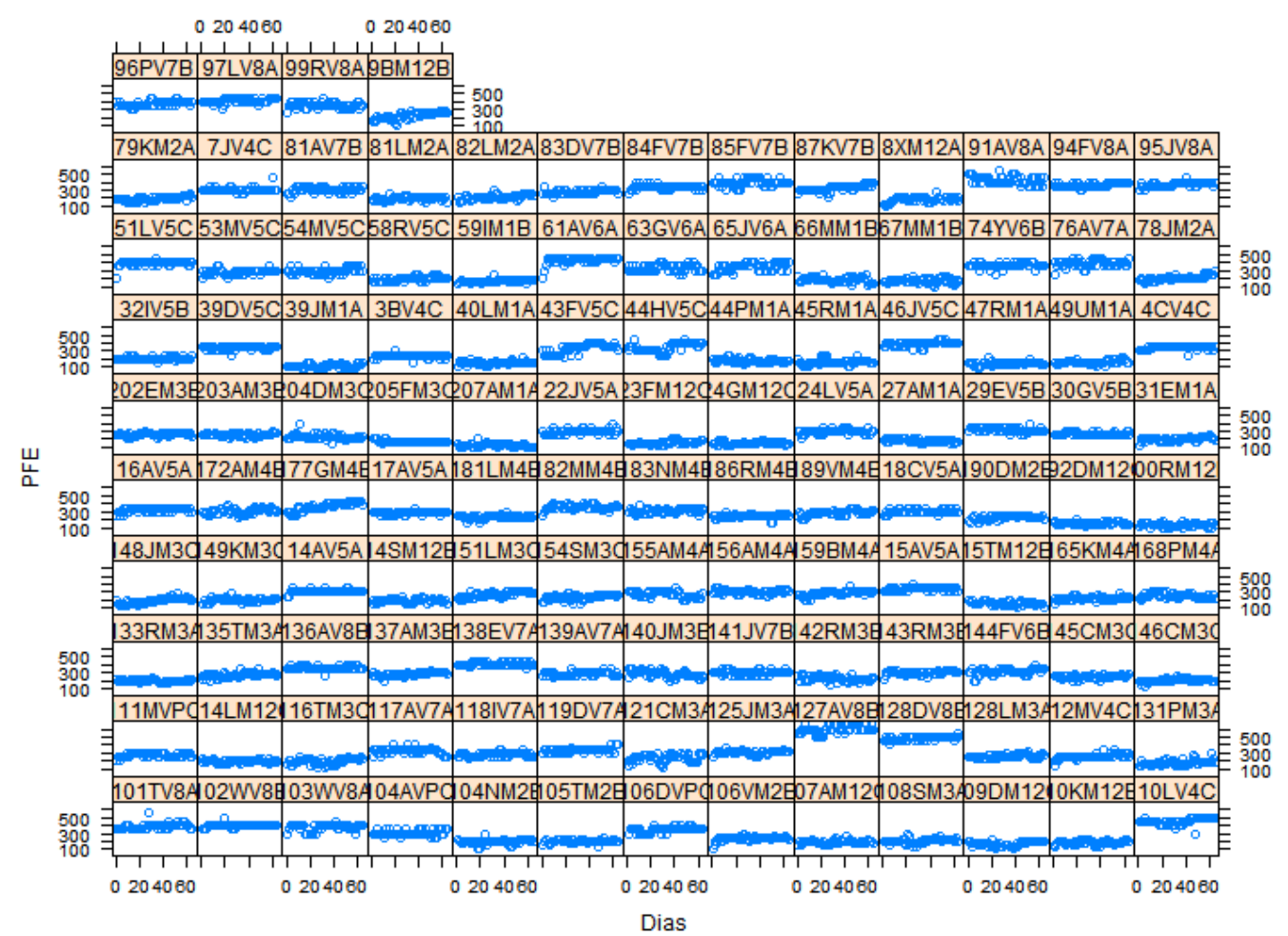

Figura A.1: Séries dos indivíduos sem a condição de asma e não expostos ao fumo domiciliar. 


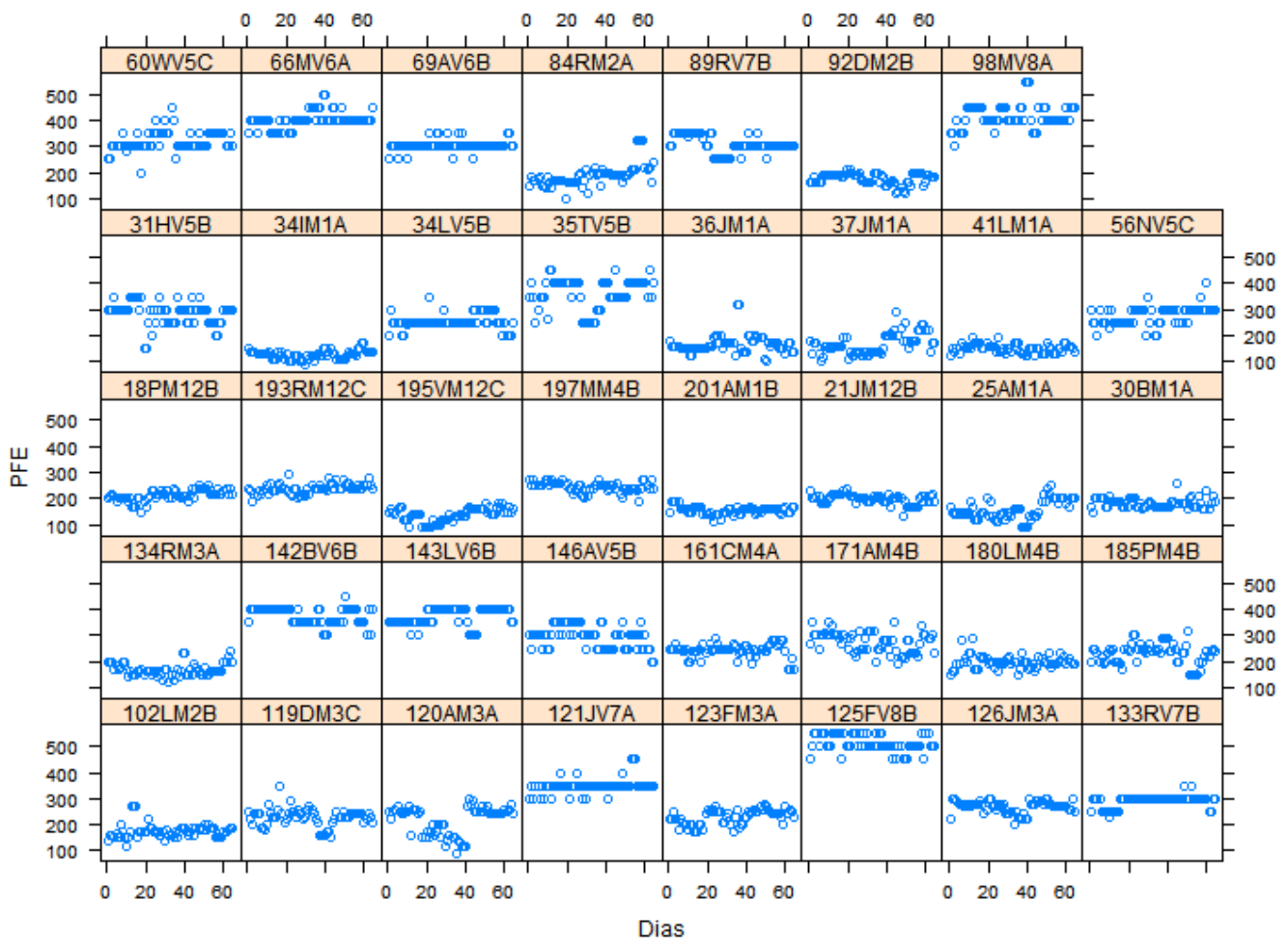

Figura A.2: Séries dos indivíduos com a condição de asma e não expostos ao fumo domiciliar.

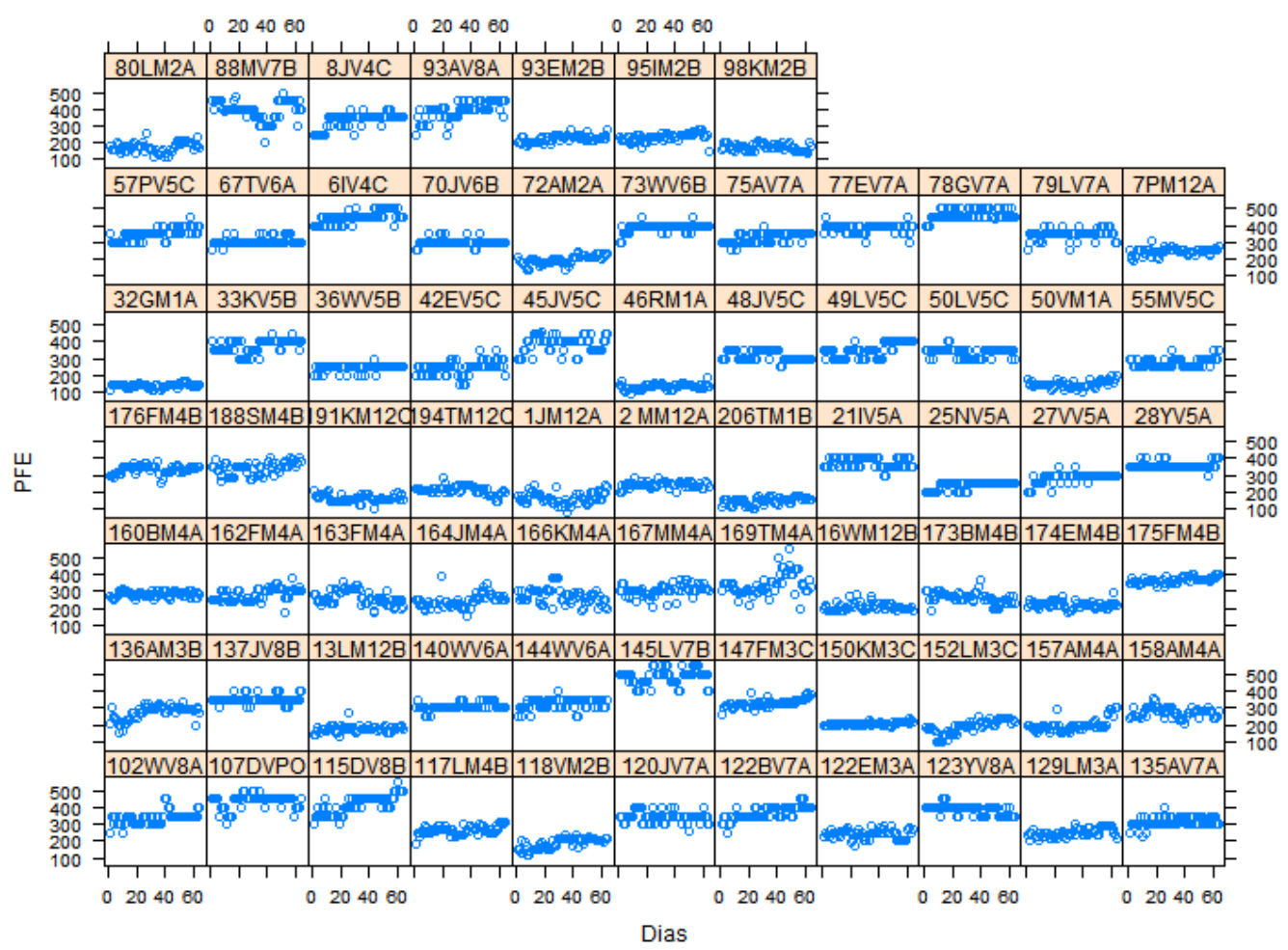

Figura A.3: Séries dos indivíduos sem a condição de asma e expostos ao fumo domiciliar. 


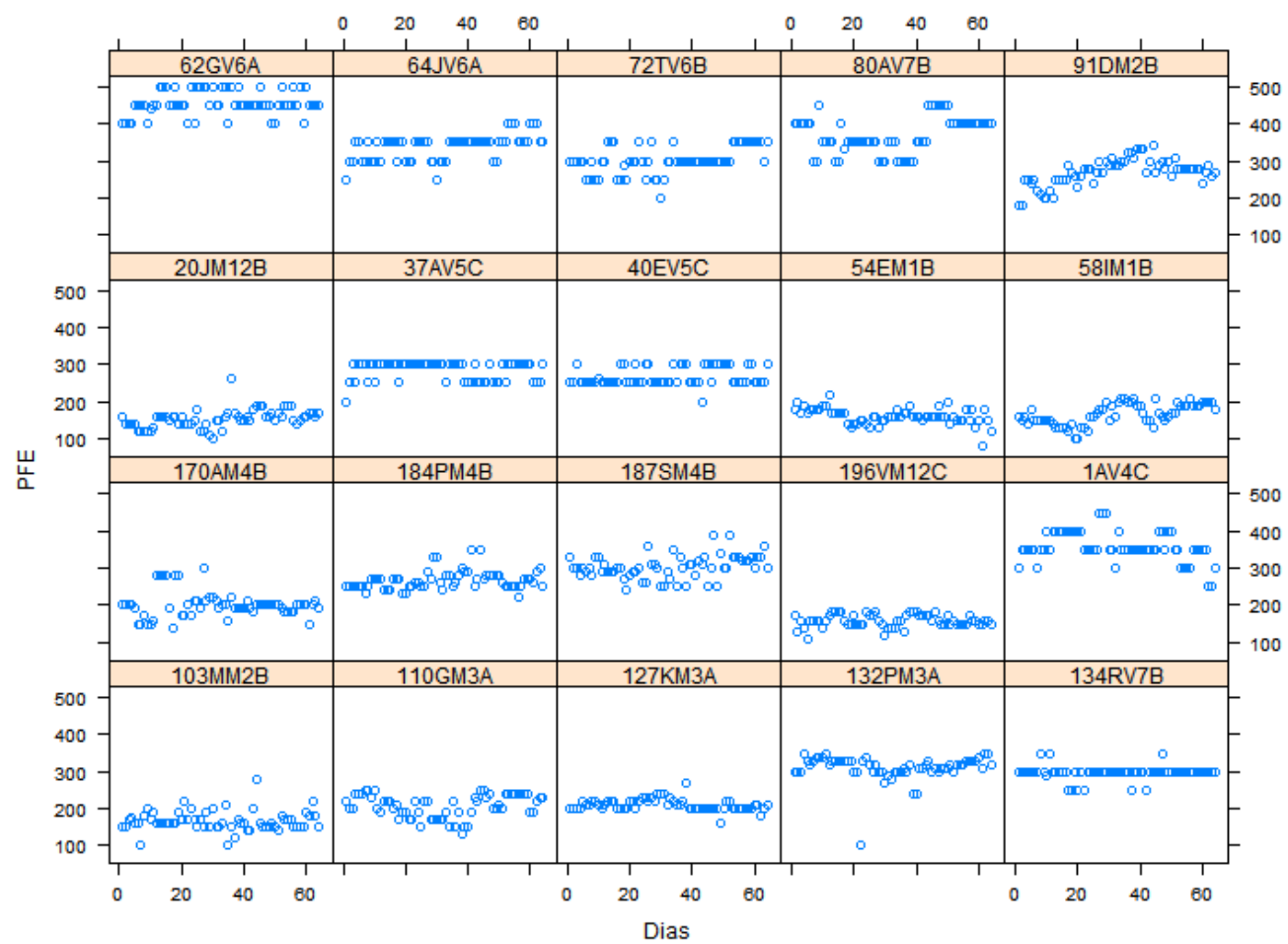

Figura A.4: Séries dos indivíduos com a condição de asma e expostos ao fumo domiciliar. 
APÊNDICE A 


\section{Referências Bibliográficas}

Abramovich(2000) Felix Abramovich. Wavelet analysis and its statistical applications. The Statistician, 49(1):1-29. Citado na pág. 1, 24

Brillinger(1981) David R Brillinger. Time series: data analysis and theory, volume 36. Siam. Citado na pág. 9, 10

Casella e Berger(2002) George Casella e Roger Berger. Statistical Inference. Duxbury, $2^{\text {a }}$ edição. Citado na pág. 39

Chiann e Morettin(1998) Chang Chiann e Pedro A Morettin. A wavelet analysis for time series. Journal of Nonparametric Statistics, 10(1):1-46. Citado na pág. 27

Chiann e Toloi(1993) Chang Chiann e Cleia Maria de Castro Toloi. Análise de variância em séries temporais. Citado na pág. 12

Chui(1992) Charles Chui. Wavelet Analysis and Its Aprocation. Academic Press, $1^{\mathrm{a}}$ edição. Citado na pág. 7

Mallat(1989) Stephane G Mallat. Multiresolution approximations and wavelet orthonormal bases of $\mathbf{L}^{2}(\mathbb{R})$. Transactions of the American mathematical society, 315(1):69-87. Citado na pág. 25

Montgomery(2017) Douglas C Montgomery. Design and analysis of experiments. John wiley \& sons. Citado na pág. 30, 31, 34

Morettin(2014) Pedro Morettin. Ondas e Ondaletas. Da Análise de Fourier à Análise de Ondaletas de Séries Temporais. Edusp, 2a edição. Citado na pág. 3, 7, 8, 9, 23

Percival e Walden(2000) Donald Percival e Andrew Walden. Wavelet Methods for Time Series Analysis. $1^{\circ}$ edição. Citado na pág. 3, 8

Pinheiro e Vidakovic(1993) A. Pinheiro e B. Vidakovic. Wavelets in functional data analysis. Citado na pág. 30, 31, 34

R Core Team(2020) R Core Team. R: A Language and Environment for Statistical Computing. R Foundation for Statistical Computing, Vienna, Austria, 2020. URL https: //www.R-project.org/. Citado na pág. 35

Roy et al.(1971) S.N. Roy, R. Gnanadesikan e J.N. Srivastava. Analysis and Design of Certain Quantitative Multiresponse Experiments. Oxford. Citado na pág. 13

Soltani et al.(2004) Skander Soltani, Patrice Simard e Daniel Boichu. Estimation of the self-similarity parameter using the wavelet transform. Signal Processing, 84(1):117-123. Citado na pág. 28 
Strachan e Cook(1998) David P Strachan e Derek G Cook. Parental smoking and childhood asthma: longitudinal and case-control studies. Thorax, 53(3):204-212. Citado na pág. 47

Whitcher(2020) Brandon Whitcher. waveslim: Basic Wavelet Routines for One-, Two, and Three-Dimensional Signal Processing, 2020. URL https://CRAN.R-project.org/ package $=$ waveslim. $\mathrm{R}$ package version 1.8.2. Citado na pág. 35 